\title{
CALIBRATION AND PERFORMANCE EVALUATION OF MINIATURE ULTRASONIC HYDROPHONES USING TIME-DELAY SPECTROMETRY
}

\author{
Lewin, Peter A
}

Published in:

I E E E Transactions on Ultrasonics, Ferroelectrics and Frequency Control

Link to article, DOI:

10.1109/T-SU.1982.31328

Publication date:

1982

Document Version

Publisher's PDF, also known as Version of record

Link back to DTU Orbit

Citation (APA):

Lewin, P. A. (1982). CALIBRATION AND PERFORMANCE EVALUATION OF MINIATURE ULTRASONIC HYDROPHONES USING TIME-DELAY SPECTROMETRY. I E E E Transactions on Ultrasonics, Ferroelectrics and Frequency Control, 29(3), 158-198. https://doi.org/10.1109/T-SU.1982.31328

\section{General rights}

Copyright and moral rights for the publications made accessible in the public portal are retained by the authors and/or other copyright owners and it is a condition of accessing publications that users recognise and abide by the legal requirements associated with these rights.

- Users may download and print one copy of any publication from the public portal for the purpose of private study or research.

- You may not further distribute the material or use it for any profit-making activity or commercial gain

- You may freely distribute the URL identifying the publication in the public portal 


\title{
1981 IEEE Ultrasonics Symposium Abstracts
}

\author{
Chicago, IL, October 14-16, 1981
}

\section{SYMPOSIUM COMMITTEE}

\section{Cochairmen}

W. D. O'Brien, $\mathrm{J}_{\mathrm{r}} \ldots \ldots \ldots \ldots \ldots \ldots$. . University of Illinois L. W. Kessler . . . . . . . . . . . . . . Sonoscan, Inc.

\section{Technical Program Committee}

M. A. Breazeale . . . . . . . . . . . University of Tennessee J. Brown . . . . . . . . . . . . . . . . Schlumberger-Doll Research P. H. Carr . . . . . . . . . . . Rome Air Development Center J.D.N. Cheeke . . . . . . . . . . . University of Sherbrooke F. Y. Cho . . . . . . . . . . . . . Motorola, GED K. L. Davis . . . . . . . . . . . Naval Research Laboratory A. J. DeVries . . . . . . . . . . . . . . Zenith Radio Corporation G. W. Farnell . . . . . . . . . . . . . . McGill University L. A. Frizzell . . . . . . . . . . . . University of Illinois S. A. Goss . . . . . . . Indianapolis Center for Advanced Research T. W. Grudkowski . . . . . . . United Technologies Research Center R. L. Gunshor . . . . . . . . . . . . Purdue University

D. L. Hecht . . . . . . . . . . . . Xerox Corporation R. S. Kagiwada . . . . . . . . TRW Defense and Space Systems L. W. Kessler . . . . . . . . . . . . . . Sonoscan, Inc. G. S. Kino . . . . . . . . . . . . . . Stanford University E. K. Kirchner . . . . . . . . . . . Teledyne MEC S. K. Kurtz . . . . . . . . . . . . . . . Clairol, Inc. J. D. Larson . . . . . . . . . . . . Hewlett-Packard Co.

E. G. Lean . . . . . . . . . . . . IBM Watson Research Center M. Levy . . . . . . . . . University of Wisconsin, Milwaukee B. R. McAvoy . . . . . . . . . . . . Westinghouse Research Labs. R. L. Melcher . . . . . . . . . . . . IBM Watson Research Center J. G. Miller . . . . . . . . . . . . . . . Washington University R. A. Moore . . . . . . . . . . . Westinghouse Electric Co. W. D. O'Brien, Jr. . . . . . . . . . . University of Illinois E. P. Papadakis . . . . . . . . . . . Ford Motor Company T. E. Parker . . . . . . . . . . . . . Raytheon Research Division R. W. Ralston . . . . . . . . . . . . . MIT Lincoln Laboratory R. L. Rosenberg . . . . . . . . . . . . . . Bell Laboratories W. H. Sachse . . . . . . . . . . . . . Cornell University J. S. Schoenwald . . . . . . . . . . Rockwell International W. R. Shreve . . . . . . . . . . . Hewlett-Packard Co. C. V. Smith, Jr. . . . . . . . . . . . . University of Texas W. R. Smith . . . . . . . . . . . . . . . Hughes Aircraft Co. R. H. Tancrell ............ Raytheon Research Division W. J. Tanski . . . . . . . . . . . . . . . S Sperry Research Center B. R. Tittman . . . . . . . . . . . . Rockwell International H. van de Vaart . . . . . . . . . . . . . Sperry Research Center R. S. Wagers . . . . . . . . . . . . . Texas Instruments, Inc. S. Wanuga . . . . . . . . . . . . . General Electric Co. R. C. Williamson . . . . . . . . . . MIT Lincoln Laboratory

Symposium Proceedings

B. R. McAvoy . . . . . . . . . . . . Westinghouse Research Labs. Student Travel Support

G. W. Farnell . . . . . . . . . . . . . . McGill University K. M. Lakin . . . . . . . . . . lowa State University R. L. Melcher . . . . . . . . . . . IBM Watson Research Center
Publicity

R. L. Gunshor . . . . . . . . . . . . Purdue University

\section{Guests' Program}

Mieke M. DeVries and Julia DesMares

The IEEE Sonics and UItrasonics Group has published the 1981 Ultrasonics Symposium Proceedings. Abstracts marked with a dagger preceding the title will not appear in the Proceedings.

Copies of the 1981 Uitrasonic Symposium Proceedings (IEEE Product Number $81 \mathrm{CH} 1689-9$ ) may be ordered through the IEEE Service Center, Order Department, 445 Hoes Lane, Piscataway, NJ 08854.

\section{G-SU PRESIDENT'S SPEAKER}

Synthesis and Recognition of Speech: Voice Communication Between Humans and Machines, J. L. FLANAGAN, Acoustics Research Department, Bell Laboratories, Murray Hill, NJ 07974.

Digital machines-computers-offer many advantages in communications and information processing. They can accomplish computations at high speed, and they can store and rapidly access vast amounts of information. Despite this sophistication, they are not adept at communicating with humans in a way humans find convenient-namely, by natural spoken language. Computers traditionally prefer the special symbols of compilers and assemblers typically communicated via a typewriter keyboard and a printed display. But, if they could be given human-like abilities for voice communication, their value and ease of use for humans would increase. And, the ubiquitous telephone would take on more of the capabilities of a computer terminal. Giving the computer a "mouth" to talk to humans draws upon the techniques of speech synthesis. Giving the computer "ears" to listen to humanspoken commands entails automatic recognition of speech. To provide computers the ability for natural language exchanges, the engineer can be guided to a large extent by the processes of human speech generation and recognition. The physics of sound generation in the human vocal tract and the signal analysis performed in the peripheral auditory mechanism are relatively well understood and can be used as design guides for synthesizers and recognizers. By contrast, the more central cerebral functions of message formulation and message comprehension are less well known, and this lack is reflected in the limitations that presently characterize machine synthesis and recognition. This talk assesses the progress of speech synthesis and recognition by computer techniques, and it outlines potential applications to new voice-communication services. Finally, it draws a perspective on foreseeable developments, and on the continuing research that will support increased sophistication in human/machine communications.

\section{SAW ELASTIC CONVOLVERS}

Chairperson: K. L. DAVIS

A-1 High Performance Saw Convolvers, M. F. LEWIS and C. L. WEST, Royal Signals and Radar Establishment, Malvern, Worcestershire, UK.

At last year's symposium a number of groups, including RSRE, reported the development of monolithic SAW convolvers with attractive 
performance characteristics: integration time $\sim 10 \mu \mathrm{s}$, band width $\sim 100$ $\mathrm{MHz}$, efficiency $\sim-75 \mathrm{dBm}$. In the intervening period we have introduced a number of refinements to the design which have improved the performance in all these primary respects, and in many secondary aspects too. At the time of writing we have produced a convolver with integration time exceeding $15 \mu \mathrm{s}$, and with efficiency exceeding -70 $\mathrm{dBm}$ over a bandwidth of $120 \mathrm{MHz}$. These improvements will be described, together with improvements to many secondary aspects such as, reduced size of the lithium niobate "chip" and its package, simpler testing and setting-up, improved suppression of unwanted selfconvolution signals, and the important aspect of improved reproducibility of the overall device characteristics.

A-2 High Performance Elastic Convolver with Extended Time-Bandwidth Product, ${ }^{1}$ I. YAO, Lincoln Laboratory, Massachusetts Institute of Technology, Lexington, MA 02173.

It is now well established that the elastic convolver is a simple, compact, and inexpensive structure which promises to perform, with realtime programmability, the matched-filtering function for waveforms of time-bandwidth product greater than 1000 . Last year at this conference, we and others described convolvers which provided the major features of programmable matched filters but without the accuracy that is needed for real applications. This paper treats the design of an improved elastic convolver which provides, for the first time, the necessary amplitude, phase, and isolation accuracy that is required in a 2200 -time-bandwidthproduct filter. This device has an efficiency of $-76 \mathrm{dBm}$. A number of second-order effects have been suppressed, most notably the self-convolution, the long-line effect of the output circuit and the phase dispersion. The emphasis of the discussion will be on 1) the dual-track selfconvolution suppression technique, 2) the simplified dual-track outputcombining circuit which minimizes the component count without sacrificing performance, and 3) and a transducer design with built-in compensatory dispersion.

${ }^{1}$ This work was supported by the Defense Advanced Research Projects Agency.

A-3 Monolithic SAW Convolvers Using Chirp Transducers, D. P. MORGAN, D. H. WARNE, P. N. NAISH, and D. R. SELVIAH, Plessey Research (Caswell) Limited, Allen Clark Research Centre, Caswell, Towcester, Northants, NN12 8EQ, UK.

For optimum efficiency, the SAW elastic convolver requires efficient generation of narrow SAW beams. Dispersive transducers can be used for this purpose, since they can have small apertures without the inconvenience of high impedances, thus avoiding the need for beam compression. In convolvers, the dispersion can be arranged to cancel. A $300-$ $\mathrm{MHz}$ transducer was designed, with $110-\mathrm{MHz}$ bandwidth and $57-\mu \mathrm{m}$ aperture. A time-bandwidth product of ten was chosen to give a radiation resistance near $50 \Omega$, as confirmed experimentally. A nonlinear chirp characteristic was used to obtain a flat amplitude response without apodisation. A $12-\mu$ s convolver with these transducers gave 110$\mathrm{MHz}$ bandwidth and $\mathrm{a}-68-\mathrm{dBm}$ bilinearity factor, using a dual-channel arrangement to give a spurious suppression of typically $35-40 \mathrm{~dB}$. Results for this and other devices will be presented. Modified chirp transducers can be used to compensate for amplitude and phase errors produced elsewhere in the convolver. A new design compensates for dispersion due to the waveguide, and experimental results will be presented.

A-4 Elastic Convolver with a Modified Multistrip Coupler Compressor, DONALD C. MALOCHA, Sawtek Inc., 2541 Shader Road, Orlando, FL. 32854 .

The elastic convolver has been successfully demonstrated as a viable device for providing a programmable matched filter function. One of the key elements in the device is the beam compressor which enhances the device efficiency. The two different beam compressors receiving the greatest development effort are the parabolic horn and the multistrip coupler compressor (MSC). The parabolic horn has the advantage of extremely easy fabrication but requires a rather long length while the MSC has a relatively short length while requiring higher resolution photolithography. This paper will present a $12-\mu$ s convolver using a modified MSC compressor. The modified coupler relaxes the resolution requirements in the coupling region, due to the change in track periodicity, while maintaining the short length and high efficiency. A slightly wider active crystal width, however, is required but is consistent with the size required for easy handling (100-200 mil crystal width). A comparison of the normal MSC and modified MSC compressor design will be provided. The convolver performance having a BT product of 1200 and input center frequency of $300 \mathrm{MHz}$ will also be presented.

A-5 Analysis of Amplitude and Phase Errors in Bilinear Convolvers, D. P. MORGAN, Plessey Research (Caswell) Limited, Allen Clark Research Centre, Caswell, Towcester, Northants, NN12 8EQ, UK.

For spread-spectrum applications, a SAW convolver must have sufficient fidelity to satisfy requirements on output pulsewidth, sidelobes, and signal-to-noise ratio. Linear device analysis is not applicable here. However, it has been shown ${ }^{1}$ that a two-dimensional frequency response can be used to characterize the device. An equivalent network is derived, in which the convolver errors are determined by a set of linear filters. An analysis is given for the effects of spatial amplitude and phase variations due, for example, to transmission-line effects. Phasecoded input waveforms are assumed. It is found that the output signal amplitude depends on the timing of the input signal, while the output noise power is a function of time. Examples are given to show the effects of several forms of amplitude and phase nonuniformity. Confirmatory experimental results are expected to be available by the time of the Symposium.

${ }^{1}$ D. P. Morgan, Electronics Lett., vol. 17, pp. 265-267, 1981.

A-6 Broadband Matching for Multiply Tapped SAW Convolvers, E. L. ADLER, Electrical Engineering Department, McGill University, Montreal, Quebec, H3A 2A7, Canada.

In long and narrow surface-acoustic-wave (SAW) convolvers the output plate electrode behaves like a lossy transmission line and when a single tap is used to extract the output signal from a convolver the amplitude and phase nonuniformities due to electromagnetic effects degrade the convolver performance. A common way of reducing the nonuniformity is to use many taps to extract the output signal. This multiple tapping solution, although it reduces the nonuniformities to acceptable levels, introduces a large number of parasitic elements into the device due to bonding pads and bonding wires and tends to lower working impedance levels and increase device insertion loss unless special output matching networks are incorporated. Using a lossy transmission line model for the convolver output plate and realistic values for the parasitic elements a circuit model for the tapped structure can be assembled and analyzed using ac circuit analysis programs. In this paper the calculated characteristics of multiply tapped convolvers are presented with particular emphasis on the impedance levels, system bandwidth, and insertion loss as a function of the number of taps. Results of the computer-aided design of appropriate matching networks are given which illustrate the trade-offs between system bandwidth, insertion loss, and passband ripple and provide useful guidelines for the design of matching networks for multiply tapped convolvers. 


\section{NDE IMAGING}

\section{Chairperson: B. R. TITTMAN}

†B-1 A New Synthetic Aperture Imaging Technique for Tomographic and Three-Dimensional Reconstructions, D. NAHAMOO and A. C. KAK, School of Electrical Engineering, Purdue University, West Lafayette, IN 47907 .

The authors recently presented a theory for a new inverse scattering technique for cross-sectional and three-dimensional imaging ${ }^{1}$. This technique was based upon the Born approximation to the wave equation and its primary highlight was that it eliminated the need for planewave insonification from the diffraction theory based approaches developed so far. In the present paper we will describe further theoretical analyses that approximately take into account the medium attenuation. A parallel theory based on Rytov approximation will also be presented. The authors will also show some new computer simulation results that are a confirmation of the theories. Finally, we will show experimental results on tissue equivalent phantoms obtained with the data collected in the transmission mode.

${ }^{1}$ D. Nahamoo and A. C. Kak, "A new technique for reconstruction from scattering fields," presented at Sixth Int. Symp. Ultrasonic Imaging and Tissue Characterization, June 1981 (Abstract appears in Ultrasonic Imaging, vol. 3, no. 2, Apr. 1981).

B-2 Real-Time Digital lmaging, ${ }^{1}$ D. K. PETERSON, S. BENNETT, and G. S. KINO, Ginzton Laboratory, Stanford University, Stanford, CA 94305 .

We have carried out careful computer and analytical treatment of errors in digital imaging systems. The effect of digitization on sidelobe levels can be predicted accurately, as well as the amplitude level of grating lobes and the effects of apodization and use of short pulses. Improvements in a three sample per wavelength digital system obtained by interpolation will be shown and demonstrations of images obtained with surface wave array transducers, a contacting longitudinal wave transducer, and a contacting shear wave transducer placed on metal blocks with internal defects present, will be given. In all cases the center frequency of operation is at $3.3 \mathrm{MHz}$.

${ }^{1}$ This work was supported by the Air Force Office of Scientific Research under Contract F49620-79-C-0217.

B-3 Reflection Tomography at 50 and $300 \mathrm{MHz},{ }^{1} \mathrm{~K}$. LIANG, G. S. KINO, and B. T. KHUR I-YAKUB, Ginzton Laboratory, Stanford University, Stanford, CA 94305.

A reflection tomographic or synthetic aperture system has been constructed for use at $50 \mathrm{MHz}$. The device employs a single transducer emitting a pulse and receiving echoes on the same transducer. In an observation of cylindrical metal or ceramic samples, the sample is rotated through $360^{\circ}$ and from $32-256$ points are used in the observations. Reconstruction is carried out on a minicomputer. Examples of images of small holes in metal and ceramic structures will be shown. A new selective backprojection technique, to improve the images of specular reflectors, will be demonstrated. It is hoped that by the time of the conference a $300 \mathrm{MHz}$ system will also be in operation.

${ }^{1}$ This work was sponsored by the Ames Research Laboratory for the Defense Advanced Research Projects Agency and the Air Force Materials Laboratory under Contract SC-81-009.

B-4 A Comparison of the Inverse Born and Imaging Techniques for Reconstructing Flaw Shapes, ${ }^{1}$ R. B. THOMPSON, K. M. LAKIN, and J. H. ROSE, Ames Laboratory-USDOE, lowa State University, Ames, IA 50011.
The inverse Born approximation is a technique for inverting ultrasonic scattering data from flaws to reconstruct the flaw shapes. Although originally derived for the case of weakly scattering flaws, it has been empirically found to produce useful results for a much wider class of objects, including voids, which violate these initial assumptions. A1though all of the physical reasons for this result are not yet understood, some important insights have been obtained by comparing the inverse Born processing algorithm to more familiar imaging approaches. In this paper the inverse Born is directly compared to wavefront reconstruction and synthetic aperture imaging approaches. The techniques are found to be essentially equivalent for isotropic media. The inverse Born is found to be less degraded by an unknown material anisotropy, but this advantage can only be gained by obtaining accurate data at frequencies lower than those usually used in imaging systems. Experimental requirements to realize this advantage are discussed.

${ }^{1}$ This work was sponsored by the Center for Advanced Nondestructive Evaluation, operated by the Ames Laboratory, USDOE, for the Defense Advanced Research Projects Agency, and the Air Force Materials Laboratory under Contract W-7405-ENG-82.

†B-6 Operating Principles and Applications of the Scanning Laser Acoustic Camera (SLAC), L. T. AUSTRHEIM, D. E. YUHAS, and L. W. KESSLER, Sonoscan, Inc., Bensenville, IL 60106.

The scanning laser acoustic camera (SLAC) is an ultrasonic imaging system which operates over the frequency range $0.8-25.0 \mathrm{MHz}$. Acoustic energy is detected by measuring the displacement of a solid plastic block surface with a stabilized Michelson interferometer. Unlike interferometer schemes previously reported, this detector is made stable against vibration and beam path instability by means of an ultrasonic pilot signal and AGC loop. A CRT is employed to display the spatial distribution of ultrasound as a two-dimensional image. The raster of information is produced by scanning the laser. One of the primary SLAC applications is ultrasonic transducer characterization in which pulsed, gated rf or $\mathrm{CW}$ operation may be employed. Applications to the testing of encapsulated electronic components and flaw detection in metals are also described.

\section{PHYSICAL ACOUSTICS I: NONLINEAR EFFECTS}

\author{
Chairperson: J. BROWN
}

C-1 [Invited] Relation of the Third-Order Elastic Constants to Other Nonlinear Quantities, ${ }^{1}$ M. A. BREAZEALE, Department of Physics, University of Tennessee, Knoxville, TN 37916.

The ordinary (second-order) elastic constants determine the velocity of ultrasonic waves in solids, and hence have been evaluated from velocity measurements in many solids under a wide range of conditions. They are ubiquitous, appearing in virtually any theory or experiment which quantitatively describes the (linear) elastic behavior of solids. The third-order elastic (TOE) constants are equally fundamental, equally ubiquitous recently, and are being measured for similar reasons: to form a basis for describing the nonlinear (elastic) behavior of solids. The TOE constants are discussed, with emphasis on cubic symmetry, with the intention of relating them to other nonlinear observables (anharmonicity, nonlinear stress-strain relationships, thermal expansion, etc.). Recent results for cubic crystals, both at room temperature and at cryogenic temperatures, are discussed as examples of current trends in solid state physics.

\footnotetext{
${ }^{1}$ This work was supported by the Office of Naval Research.
}

C-2 [Invited] Nonlinear Ultrasonic Wave Propagation in Biological Materials, ${ }^{1}$ F. DUNN, W. K. LAW, and L. A. FRIZZELL, Bjoacoustics Research Laboratory, University of Illinois, Urbana, IL 61801. 
The nonlinearity parameter $\mathrm{B} / \mathrm{A}$ has been determined for tissue models (aqueous solutions of biological macromolecules) and for excised tissues from measurements, of the fundamental at the source and the second harmonic as a function of distance from the source. The general demeanor of the tissue model results is that $\mathrm{B} / \mathrm{A}$ increases nearly linearly with solute concentration but, for a fixed concentration, is relatively insensitive to the molecular weight of the solute molecules. Whole mammalian blood, whose dry weight consists largely of protein, exhibits a $B / A$ value essentially that of protein solutions of the same dry weight concentration. Excised mammalian liver yields a $B / A$ value significantly greater than blood, and homogenization of the tissue substantially reduces the $B / A$ value. These findings suggest that the solutions become more nonlinear as the spacing distance between solute molecules decreases and that tissue architecture contributes to nonlinear behavior. It is felt that details of nonlinear ultrasonic propagation in living systems should contribute to further improvement of clinical diagnostic and therapeutic procedures.

${ }^{1}$ This work was supported by NSF and NIH.

C-3 Saw Second Harmonic Fields Nonlinearly Produced by Spatially Limited Beams, ${ }^{1}$ A. ALIPPI, A. PA LMA, L. PALMIERI, G. SOCINO, and E. VERONA, Istituto di Acustica "O. M. Corbino, CNR, 00189 Rome, Italy.

Second harmonic waves produced in surface acoustic wave nonlinear propagation have a field pattern which is locally bound to the diffraction field of the fundamental wave and to the evolution of the second harmonic field generated up to the considered point. A theoretical model is proposed which considers each plane wave in the momentum spectrum of the fundamental wave to generate a second harmonic wave by interaction with all the other spectral components: the field is then obtained by the integration of all the partial contributions. Second harmonic fields were detected by means of a high resolution phase contrast optical probing technique. Transversal patterns are presented of the second harmonic field produced in $y z$ - $\mathrm{LiNbO}_{3}$ crystals by fundamental beams of different directivity and a comparison is performed with the theoretical profiles.

${ }^{1}$ This work was partially supported by the CNR-NSF program.

C-4 Relative Slope Invariance of Velocity-Stress and Strain-Stress Curves, J. H. CANTRELL, JR. and E. J. CHERN, ${ }^{1}$ NASA Langley Research Center, Hampton, VA 23665.

Recent experiments have shown that for a wide variety of isotropic materials the stress dependence of the "natural" velocity, the strain, and the true velocity are characterized by "linear" curves whose relative slopes are to a good approx imation material independent. For example, the ratio of slopes of the "natural" velocity curve to the strain curve is approximately $3: 1$ for most materials measured. We derive these experimental results from the thermoelastic theory in which the slope ratios are expressed in terms of combinations of second- and thirdorder clastic coefficients (TOEC) and the compliance coefficients. The slope ratios are strongly influenced by the sign of the TOEC which are doninantly negative for most materials. Materials with dominantly positive TOEC exhibit anomalous behavior. For materials of lower crystalline symmetry the compliance coefficients are shown to be equal to the free Young's modulus and result in approximately the same slope ratios as for isotropic materials.

${ }^{2}$ Research Associate, College of William and Mary, Williamsburg, VA 23185 .

\section{SAW-BASED SIGNAL PROCESSING}

\section{Chairperson: T. W. GRUDKOWSKI}

D-10 [Invited] Digital Processors Using SAW Devices, ${ }^{1}$ H. GAUTIER, M. CHOMIKI, P. LEDU, Thomson-CSF DASM Cagnes/Mer-06802-
France, P. ANTHOUARD, Thomson-CSF AVS/RCM Malakoff-92240France.

To build digital input/output processors showing both compactness and high computation speed, one can couple SAW modules to digital interfaces. The analog processing heart can indeed process $10^{7}$ to $10^{9}$ points per second and the full processor will exhibit similar performances provided adequate interfacing and digital circuits are used. The purpose of this paper is to demonstrate the compatibility of the technologies to be coupled, the feasibility and the advantages of the resulting hybrid processors. We first compare the performances of the necessary acoustic and electronic components. We then describe several particular examples of realizations which employ acoustic Fourier analyzers and convolvers. And we discuss the performances the complexity and the dissipated power of the module and of those to be developed.

${ }^{1}$ Some of the modules described were developed under DRET contracts.

D-2 Compact Multiple-Channel SAW Sliding-Window Spectrum Analyzer, ${ }^{1}$ D. R. ARSENAULT and V. S. DOLAT, Lincoln Laboratory, Massachusetts Institute of Technology, Lexington, MA 02173.

A spectrum analyzer consisting of twelve identical SAW chirp-Fourier transform circuits was developed to process twelve parallel-beam Doppler-shifted $\mathrm{CW}$ returns from an airborne infrared laser radar. System requirements are for a resolution of $1.6 \mathrm{~km} / \mathrm{h}$ over a velocity spread of $\pm 100 \mathrm{~km}$. At the $10.6-\mu \mathrm{m}$ laser wavelength, this translates to an $84-\mathrm{kHz}$ frequency resolution with $10-\mathrm{MHz}$ analyzer bandwidth. These requirements and the need for $>50 \mathrm{~dB}$ dynamic range with sidelobes $>25 \mathrm{~dB}$ down are well-matched to the properties of moderate time-bandwidthproduct RAC devices. A major constraint was to minimize size, power consumption, and cost. The prototype 12-channel subsystem is contained within a $3^{\prime \prime} \times 8^{\prime \prime} \times 10^{\prime \prime}$ volume and requires approximately 25 W. The optimized configuration with circuits developed for this system will be discussed along with its performance characteristics.

'This work was supported by the Department of the Air Force.

D-3 SAW Based Spread Spectrum Modem Combining Chirp and Pseudonoise Coding, ${ }^{1}$ M. KOWATSCH, J. LAFFERL, and F. J. SEIFERT, Abteilung für Angewandte Elektronik, Institut für Allgemeine Elektrotechnik und Elektronik, Technische Universität Wien, Gußhausstraße 27-29, A-1040 Vienna, Austria.

Chirp pulse compression using SAW dispersive delay lines offers an attractive means for encoding digital data in low cost reliable spread spectrum systems. However, chirp modems suffer from the limited variability of the parameters defining the linear FM signal. These restrictions can be overcome by combining chirp modulation with the highly flexible digital pseudonoise (PN) phase shift keying (PSK), maintaining the superior Doppler performance of the linear chirp. ${ }^{1}$ The paper reports on a modem designed for data transmission at 100 kbps. Chirps with $T=10 \mu \mathrm{s}$ and $B=16 \mathrm{MHz}$ are modulated by different 127 bit $m$-sequences. A large number of programmable signals is available even with these short codes, because a cyclic shift of the periodic $m$-sequence relative to the chirp starting point generates a new waveform. The receiver code synchronization and tracking is explained and experimental results are presented.

\footnotetext{
${ }^{1}$ This work was supported by the Austrian Ministry of Defense.

${ }^{2}$ M. Kowatsch, F. J. Seifert, and J. Lafferl, "Comments on transmission system using pseudonoise modulation of linear chirps," IEEE Trans. Aerosp. Electron. Syst., vol. AES-17, pp. 300-302, Mar. 1981.
}

D-4 Effect of Amplitude Limiting on Pulse Compression of Overlapping Chirps, ${ }^{1}$ J. LAFIERL, M. KOWATSCH, and F. J. SEIFERT, 
Abteilung für Angewandte Elektronik, Institut für Allgemeine Elektrotechnik und Elektronik, Technische Universität Wien, Gußhausstraße 27-29, A-1040 Vienna, Austria.

In analog or digital encoded linear FM SAW spread spectrum systems employing high data rate overlapping chirp operation, amplitude variations arise in the composite IF signal. Efficient utilization of the transmitter power capability generally implies amplitude limiting of the transmitted waveform. The paper investigates the influence of limiting on the compression gain and dynamic range of an analog pulse position modulated chirp carrier system. ${ }^{2}$ Computer simulation is in good agreement with experimental results, which were obtained in a modem using SAW dispersive delay lines with $T=10.8 \mu$ s and $B=16.4 \mathrm{MHz}$. Measured data are given on the distortions of the demodulated signal in dependence on limiter parameters. Under limiting conditions a linear dynamic range in the order of $40 \mathrm{~dB}$ is obtainable, if zero-crossing detection is applied in the receiver.

\footnotetext{
${ }^{1}$ This work was supported by the Austrian Ministry of Defense.

${ }^{2}$ M. Kowatsch, J. Lafferl, F. J. Seifert, and H. R. Stocker, "Analog encoded chirp transmission system using surface acoustic wave filters," IEEE Trans. Sonics Ultrason., vol. SU-27, pp. 355-359, Nov. 1980.
}

D.5 A New Iterative Transform Algorithm Based on a Modular SAWCFT Unit, P. TORTOLI, G. F. MANES, and C. ATZENI, Istitu to di Elet tronica, Università di Firenze, via S. Marta, 3, Firenze, Italy.

SAW Chirp Fourier transform processors (CFTP) have been proposed as an effective replacement of digital units for Fourier transform operating in digital systems, when proper $A / D$ D/A interfaces are provided. As the typical execution time ( $\left.T_{a}\right)$ of SAW-CFTP's is fixed and ranges from $\mu$ s to some tens of $\mu$ s, an increasing transform size $(N)$ requires a correspondingly increasing data compression when intrinsic CFTP data rate $N / T_{a}$ exceeds the overall system data rate. This results in overloading the A/D D/A interfaces independently of general system requirements and prevents from an efficient utilization of SAW unit to be attained for high transform sizes. A new technique for arbitrary extending the execution time $\left(M T_{a}\right)$ and transform size $(N \cdot M)$ of a SAWCFTP is discussed and demonstrated, based on using a single transform unit in an iterative algorithm, consisting of $2 M$ sequential transforms, each of execution time $T_{a}$ and size $N$. A significant extension of the SAW-CFTP interface capability to digital systems is then obtained, while removing any arbitrary speed requirement of $A / D$ and $D / A$ interfaces.

\section{NDE FLAW CHARACTERIZATION}

\section{Chairperson: J. M. RICHARDSON}

$\div$ E-10 The Crack Tip, Viewed as a Secondary Source of Radiation, ${ }^{1}$ B. R. TITTMANN and L. A. AHLBERG, Rockwell International Science Center, Thousand Oaks, CA 91360; and A. MAL, Department of Mechanics and Structures, University of California, Los Angeles, CA 90024 .

Experimental and theoretical results are presented on the angular and frequency dependence of the longitudinal and shear wave radiation patterns from the tip of a simulated surface crack irradiated by Rayleigh waves. These data form the basis for determining the depth of the crack tip below the specimen surface and the acoustic path length along the face of the crack to its tip. This approach is demonstrated on a true fatigue crack grown by cycling a specimen of $2024 \mathrm{Al}$ under uniaxial tension.

'This work was in part sponsored by the Center for Advanced Nondestructive Evaluation, operated by the Ames Laboratory USDOE, for the Defense Advanced Research Projects Agency, and the Air Force Wright Aeronautical Laboratories/Materials Laboratory under Contract W-7405-ENG. 82. $\left\lceil\right.$ E-2 Sizing of Edge Cracks in a Plate Using SH Waves, ${ }^{1}$ C. M. FORTUNKO, Fracture and Deformation Division, National Bureau of Standards, Boulder, CO 80303; and Z. ABDULJABBAR and S. K. DATTA, Department of Mechanical Engineering, University of Colorado, Boulder, CO 80303 .

An integral equation method is employed to investigate the scattering of horizontally polarized shear waves ( $\mathrm{SH}$ waves) by two-dimensional, edge cracks in a plate. The equation is solved numerically for the amplitudes and phases of the scattered SH plate modes. The results, which reveal several important features of the scattered SH wave field, are compared with experimental data. Electromagnetic-acoustic transducers (EMAT's), operating at $454 \mathrm{kHz}$, are used to detect only the two lowest order $\mathrm{SH}$ modes $\left(\mathrm{SH}_{0}\right.$ and $\left.\mathrm{SH}_{1}\right)$ that are back-scattered by thin machined notches in a $1.59-\mathrm{cm}$ steel plate. The EMAT's reject the three higher order $\mathrm{SH}$ modes that can propagate. It is demonstrated that, by using multimoded $\mathrm{SH}$ wave probing signals, it is possible to distinguish edge cracks from interior cracks. Furthermore, it is possible to determine their size. The results are applicable to sizing an important category of weld defects at long and medium wavelengths.

${ }^{1}$ This work was supported, in part, by the DoT, Office of Pipeline Safety.

E-3 Long Wavelength Measurements of Surface Cracks in Silicon Nitride, ${ }^{1}$ J. J. TIEN, K. LIANG, and B. KHURI-YAKUB, Ginzton Laboratory, Stanford University, Stanford, CA 94305; and D. MARSHALL and A. EVANS, University of California, Berkeley, CA.

A low-frequency theory for scattering from surface cracks has been developed. For the case of half-penny shaped surface indentation cracks, the reflection coefficient of a Rayleigh wave incident on a crack can be related to the crack size. The fracture stress of a sample containing such a crack may then be predicted from the crack size. The change in the reflection coefficient that occurs as a sample is stressed to fracture has also been measured for polished silicon nitride plate specimens and the acoustic measurements correlated with the crack extension behavior preceding final failure. In addition, experimental studies have been made on machined silicon nitride rod and plate specimens. Estimated values of the crack size and fracture stress due to machining induced cracks obtained by extrapolating the crack extension behavior observed for polished plate specimens to the rod geometry and to long cracks were found to be within 20 percent of the true values.

${ }^{1}$ This work was sponsored by the Ames Research Laboratory for the Defense Advanced Research Projects Agency and the Air Force Materials Laboratory under Contract SC-81-009 and the Office of Naval Research under Contract N00014-78-C-0283.

E-4 Improvements in Flaw Detection and Sizing in Austenitic Stainless Steel Weldments, ${ }^{1}$ C. FORTUNKO and R. K. ELSLEY, Rockwell International Corporation, Science Center, Thousand Oaks, CA 91360.

Nondestructive inspection of austenitic stainless steel weldments presents special problems because anisotropic grain growth occurs along the direction of thermal gradient during welding. For incident shear vertical (SV) or longitudinal $(L)$ waves, this results in unwanted reflections from the base metal-weld metal interface and in complicated propagation within the weld region. It is shown that significant improvements in crack detection and sizing can be obtained by the use of horizontally polarized shear (SH) waves launched by EMAT transducers. For selected angles of incidence, SH waves can pass through the base metal-weld metal interface without partial reflection, yielding cleaner flaw signals. The use of wavelengths comparable to or longer than the flaw size is advantageous because it reduces the influence of nonuniform texture at the interface. The low conductivity and nonmagnetic nature of austenitic stainless steel reduce the efficiency of the EMAT transducers used to launch the SH waves. To compensate for this, digital signal processing techniques including signal averaging 
and synthetic aperture processing are used to improve the signal-tonoise ratio and directivity of the EMAT transducers.

${ }^{1}$ This work was supported by the National Bureau of Standards and the Electric Power Research Institute (Contract T 110-2).

E-5 The Role of Creep Rays in the Scattering of Elastic Waves from Spheroidal Cavities ad Inclusions in Solids, ${ }^{1}$ F. COHEN-TENOUDJI, ${ }^{2}$ L. AHLBERG, B. R. TITTMANN, Rockwell International Science Center, Thousand Oaks, CA $91360 ;$ J. L. OPSAL, Lawrence Livermore Laboratory, Livermore, CA 94550 ; V. V. VARADAN, Ohio State University, Columbus, OH 43210; G. QUENTIN, Université Paris VII, 75221 Paris/France.

Theoretical and experimental results are presented for the scattering of elastic waves from cavities (oblate spheroids with $4: 1,2: 1$ aspect ratios, spheres, and a prolate spheroid with $2: 1$ aspect ratio) and a Tungsten Carbide inclusion all embedded in Ti-alloy. Attention is focussed on the role of the creep rays in the time and frequency domains. They are found to have velocities 10 percent less than that of the longitudinal wave in the host. For nonspherical flaw geometries, two sets of creep rays have been identified experimentally and used to determine flaw shape and size.

\footnotetext{
${ }^{1}$ This work was in part sponsored by the Center for Advanced Nondestructive Evaluation, operated by the Ames Laboratory USDOE, for the Defense Advanced Research Projects Agnecy and the Air Force Wright Aeronautical Laboratories/Materials Laboratory under Contract W-7405-ENG. 82.

${ }^{2}$ Permanent address: Université Paris VII, 75221 Paris/France.
}

$\dagger$ E-6 Scattering from Inclusions in the Time Domain, J. M. RICHARDSON and B. R. TITTMANN, Rockwell International Science Center, Thousand Oaks, CA 91360.

It is known that the scattering of elastic waves from spherical inclusions has a complex description in the frequency domain, but a relatively simple one in the time domain. In the time domain the description, i.e., the impulse response function (IRF), involves typically a number of prominent sharp pulses which evidently correspond to the stationary solutions of the elastodynamic Fermat's principle. Since a pulse in the time domain at $t=\tau$ corresponds to a contribution proportional to $\exp (-j \omega \tau)$ in the frequency domain, it seems more appropriate to regard the roles of time and frequency as reversed relative to their roles in the resonance picture. In the present paper the following topics will be covered: a) IRF's for various spherical inclusions (with the void regarded as a special case) in $\mathrm{Si}_{3} \mathrm{~N}_{4}$ and $\mathrm{SiC}$, (b) time delays, magnitudes, and polarities of pulses, c) approximate reconstruction of frequency-domain scattering amplitudes from the properties of prominent pulses in the IRF's, and d) extraction of pulse properties from raw wave forms.

\section{PHYSICAL ACOUSTICS II: SURFACE ACOUSTIC WAVES}

\section{Chairperson: J.D.N. CHEEKE}

\section{F-1 Leaky Rayleigh Waves at a Layered Half-Space Fluid Interface,} D. E. CHIMENTI, AFWAL Materials Laboratory, Wright-Patterson AFB, OH 45433 and A. H. NAYFEH, ${ }^{1}$ Systems Research Laboratories, Dayton, OH 45440 .

Experimental and theoretical results on the nonspecular reflection of finite acoustic beams incident at and near the Rayleigh angle onto a fluid-solid interface in contact with an elastic layer are presented. Measurements with Gaussian-shaped acoustic beams have been performed on the reflected field amplitude distribution and surface wave-speed dispersion for loading and stiffening layers where the fluid medium is water. It is shown that existing theory can be utilized to explain the results by constructing and solving boundary condition equations for the Rayleigh wave pole appropriate for the lossless layered half-space with the fluid. Excellent agreement with the exact treatment is observed in most aspects of the measurements. In the model calculation the imaginary part of the Rayleigh wave pole is found to display an unexpected minimum at a value of layer thickness over wavelength where the real part corresponds nearly to the transverse wave-speed in the layer.

${ }^{1}$ Consultant-Permanent address: Department of Aerospace Engineering and Applied Mechanics, University of Cincinnati, $\mathrm{OH} 45221$.

$\uparrow$ F-2 Electron-Phonon SAW Attenuation of $\mathrm{Nb}_{3} \mathrm{Sn} \mathrm{ON} \mathrm{LiNbO}{ }_{3},{ }^{1} \mathrm{~S}$. C. SCHNEIDER, H. P. FREDRICKSEN, and M. LEVY, Department of Physics, University of Wisconsin-Milwaukee, Milwaukee, WI 53201.

The electron-phonon attenuation of SAW waves traveling in a layered film-substrate combination can be calculated by including a loss term due to viscous damping in the equation of motion for the film. In the limit of large $k h$, where $k$ is the phonon propagation vector and $h$ is the film thickness, the attenuation approaches the "Viktorov" limit where the SAW attenuation is primarily determined by the transverse bulk attenuation. For most film-substrate combinations analyzed by the analy tic continuation method, the attenuation rises monotonically to the high $k h$ limit from below. For a $\mathrm{Nb}_{3} \mathrm{Sn}$ film on a $\mathrm{LiNbO}_{3}$ substrate, however, the calculated attenuation overshoots the high $k h$ limit by $\sim 30$ percent at $k h=2.5$ and then relaxes to this limit from above. In addition, there is a small local maximum in the attenuation at $k h=$ 0.5 . This anomalous behavior may be produced by the fractional change of "longitudinal" and "transverse" motion that is present as the film thickness increases. An analysis of these individual contributions will be presented and an attempt will be made to predict other film/ substrate combinations which will exhibit this attenuation behavior.

${ }^{1}$ Research supported by the Air Force Office of Scientific Research under Grant AFOSR 81-0002.

F-3 Influence of $\mathrm{SiO}_{2}-\mathrm{Si}$ Interface States on $\mathrm{SAW}$ Transduction in a $\mathrm{SiO}_{2}-\mathrm{Si}$ Layered Medium Covered with a Piezoelectric Overlay, A. VENEMA, Department of Electrical Engineering, Delft University of Technology, 4 Mekelweg, 2628 CD Delft, The Netherlands.

A detailed treatment dealing with the $\mathrm{SiO}_{2}-\mathrm{Si}$ interface-state influence on SAW transduction in three-layered media, with a silicon substrate (e.g., $\mathrm{ZnO}-\mathrm{SiO}_{2}-\mathrm{Si}$ and $\mathrm{CdS}-\mathrm{SiO}_{2}-\mathrm{Si}$ layered media), is presented. An interface-state network model is used to predict the degradation of SAW transduction performance given the frequency range, silicon conductivity, oxide thickness, and surface-state density.

F-4 Effect of Nickel Film Thickness on the Attenuation of SAW, ${ }^{1}$ H. YOSHIDA, G. T. LEE, M. L. XU, and M. LEVY, Physics Department, University of Wisconsin-Milwaukee, Milwaukee, WI 53201.

SAW delay lines on quartz substrates covered with $\mathrm{Ni}$ exhibit a large attenuation change for a small magnetic field. This attenuation behavior appears to depend upon the conditions of sample preparation. The attenuation of the SAW delay line has been measured during deposition of the $\mathrm{Ni}$ film to attempt to correlate deposition conditions with the subsequent magnetic field dependent attenuation. The insertion loss versus thickness during deposition exhibits a sharp maximum at $\sim 12 \AA$ and a broad maximum near $200 \AA$. The sharp maximum may be associated with resistive losses due to currents in the film produced by the piezoelectric fields accompanying the SAW. The broad maximum appears to be associated with magneto-elastic coupling of the SAW with the Ni film. The magnetic field dependent attenuation will be presented 
for films with thickness of $100 \AA, 200 \AA, 250 \AA$, and $300 \AA$. Some of the deposition parameters which appear to be important will be discussed.

${ }^{1}$ Research supported by the National Science Foundation under Grant ECS-8001773.

F-5 Waves Guided by a Thin Viscoelastic Layer Between Elastic Solids, R. O. CLAUS and R. T. ROGERS, Department of Electrical Engineering, Virginia Polytechnic Institute and State University, Blacksburg, VA 24061.

The propagation of ultrasonic waves guided along a viscoelastic layer which separates two dissimilar elastic solid half-spaces is described. Layer and half-space wave potential solutions to the general eight-byeight determinant equation obtained by satisfying stress and displacement boundary conditions at both layer-substrate interfaces are determined as functions of layer thickness and elastic constants. For the limiting cases of rigid and soft bonding by a layer thin compared to acoustic wavelength, Stoneley and independent Rayleigh wave characteristic equations, respectively, result. ${ }^{2}$ For combinations of layer rigidity and wave-length that correspond to guided wave propagation, external cyclic loading of the layer produces mechanical hysteresis and a resulting dynamic change in bond properties. ${ }^{3}$ Experimental measurements of the observed velocity hysteresis in an aluminum-polymer adhesive-aluminum system as described and potential applications in nondestructive bond characterization are indicated.

${ }^{1}$ Work supported by National Science Foundation.

${ }^{2}$ S. Rokhlin, M. Hefets, and M. Rosen, J. Appl. Phys, vol. 51, p. 3579,1980 .

${ }^{3}$ D. A. Lee and D. M. Corby, IEEE Trans. Sonics Ultrason., vol. SU-24, p. 206,1977 .

F-6 Applicability of Current Theories of Akhieser Damping in Solids, M. NANDANPAWAR and S. RAJAGOPALAN, Acoustic Laboratory, Department of Physics, Nagpur University, Nagpur 440010, India.

In the present work, we have formulated an expression for Akhieser loss satisfying Barret's criteria. ${ }^{1}$ The nonlinearity parameter in this expression vanishes if all the indjvidual mode Gruneisen numbers are equal; the loss remains finite even for shear waves for which the average of Gruneisen numbers is zero. The expression involves the specific heat rather than the thermal energy. It is thus free from drawbacks suffered by the earlier expressions for Akhieser loss. We have used the present formulation for the estimation of attenuation in three semiconductors ( $\mathrm{Ge}, \mathrm{Si}, \mathrm{InSb}$ ) at room and liquid nitrogen temperatures. Comparison with experiment shows that Mason's theory, in spite of all the objections raised against it, gives the best fit with experimental data and the agreement does not appear to be fortuitous as held by Barret.

'H. H. Barret and M. G. Holland, Phys. Rev., vol. 1, p. 2538, 1970.

\section{NDE TRANSDUCERS}

\section{Poster Session}

G-1 Contacting Transducers and Transducer Arrays for NDE, ${ }^{1} R$. BAER, A. R. SELFRIDGE, B. T. KHURI-YAKUB, and G. S. KINO, Ginzton Laboratory, Stanford University, Stanford, CA 94305 and J. SOUQUET, Varian Associates, 611 Hansen Way, Palo Alto, CA 94303.

We have designed and built longitudinal and shear wave contacting arrays for the nondestructive evaluation of aluminum. Both arrays have 32 elements and operate at a center frequency of $3 \mathrm{MHz}$. The round trip insertion loss of each array element is about $14 \mathrm{~dB}$, with a fractional bandwid th of 30 percent. Side drilled holes in an aluminum block are imaged using these arrays in a real time synthetic aperture imaging system. Following a suggestion of H. D. William of the CEGB (England), the coupling problem between the arrays and the aluminum block under test has been solved by using natural bee honey. Our initial experiments with bulk and shear wave transducers in the frequency range of $3 \mathrm{MHz}$ indicate that honey is a far superior couplant $(20-30$ dB) to any of the other available couplants.

${ }^{1}$ This work was supported by the Air Force Office of Scientific Research under Contract F49620-79-C-0217.

G-2 The Twin Magnet EMAT Configuration for Exciting Horizontally Polarized Shear Waves, ${ }^{1}$ J. F. MARTIN and R. B. THOMPSON ${ }^{2}$ Rockwell International Science Center, Thousand Oaks, CA 91360.

A new EMAT configuration is presented for generating horizontally polarized, ultrasonic shear waves. Previous EMAT configurations to excite these waves made use of periodically polarized, permanent magnets and spatially uniform time harmonic current sheets to induce surface tractions oriented perpendicular to the direction of periodicity. Horizontally polarized shear $(\mathrm{SH})$ waves were launched at an angle determined by the product of magnet periodicity and drive frequency. Although suitable for a variety of applications, these periodic permanent magnet EMATs suffer from the problems that 1) bias fields are limited to those attainable with available permanent magnet materials, and 2) unidirectional operation is not easily achieved. An alternate configuration to generate SH waves, utilizing a modified meander coil and a twin magnet structure, is presented which overcomes these problems. Included are discussions of efficiency, radiation patterns, and application to the inspection of railroad rails.

${ }^{1}$ This work was supported by the Independent Research and Development Program of Rockwell International and the Federal Railroad Administration under Contract DTFR5 3-80-C-00121.

${ }^{2}$ Permanent Address: Applied Science Center, Ames Laboratory, lowa State University, Ames, IA 50011.

G-3 A New Nonlinear FM Coded SAW EMAT for Nondestructive Testing of Materials, F. HAUSER, G. R. DUBOIS, and V. M. RISTIC, Department of Electrical Engineering, University of Toronto, Toronto, Ontario, Canada M5S $1 \mathrm{A4}$; and H. LICHT, A tomic Energy of Canada Ltd., Chalk River, Ontario, Canada KOJ 1 Jo.

A new nonlinear FM function has been applied to the design of pulse compression EMAT's used in surface crack detection and SAW velocity variation measurements. The experimentally obtained compressed pulse $S / N$ ratio is $10 \mathrm{~dB}$ and the time resolution is $500 \mathrm{~ns}$.

G-4 Ultrasonic Transducer with Gaussian Radial Velocity Distribution, P. S. ZERWEKH and R. O. CLAUS, Department of Electrical Engineering, Virginia Poly technic Institute and State University, Blacksburg, VA 24061 .

In materials evaluation applications requiring the interrogation of the modified far field pattern of an ultrasonic transducer it is desirable to use a transduces which produces a beam with a Gaussian profile, ${ }^{2,3} \mathrm{~A}$ transducer with a velocity profile Gaussian as a function of radius and independent of angle is described. The transducer has been constructed by depositing a circularly symmetric metallic multielectrode array on a $19.1 \mathrm{~mm}$ diameter $x$-cut quartz disk. Each electrode is independently connected to an impedance network optimized to produce the Gaussian distribution with less than two percent maximum error. A computeraided electrode design and calibrated three-dimensional interferometric optical measurements of the far field distribution are presented.

'Work was supported by NSF and NSA.

${ }^{2}$ K. von Haselberg and J. Krautkrämer, Acustica, vol. 9, p. 359, 1959.

${ }^{3}$ F. D. Martin and M. A. Breazeale, J. Acoust. Soc. Amer, vol. 49, p. 1668,1971 . 
G-6 Design of a Linear Array of UItrasonic Transducers for Nondestructive Evaluation, ${ }^{1}$ J. C. SOUMAGNE, G. R. DUBOIS, and V. M. RISTIC, Department of Electrical Engineering, University of Toronto, Toronto, Canada M5S 1A4.

A schlieren system using a pulsed light source, consisting of a laser and an electrooptic modulator, synchronized with the acoustic excitation is used to visulaize short acoustical pulses created by a linear array of transducers. This system permits the characterization of the performance of the array, i.e., beam size, steering and focusing. The linear array produced, uses a multilayer scheme for adaptation of the PZT high acoustic impedance to water, and a high impedance lossy backing resulting in efficient and wideband transducers. The experi mental results, (electrical impedance, impulse response and efficiency) are compared with the theoretical results given by the transmission line model of Krimholtz, Leedom, and Matthaei.

'This work was supported by the National Research Council of Canada.

† G-7 Optimal Selection of Measurements in Ultrasonic Nondestructive Testing, ${ }^{1}$ J. M. RICHARDSON, Rockwell International Science Center, Thou sand Oaks, CA 91360.

An important, essentially unsolved, problem in the ultrasonic nondestructive testing of materials is the optimal selection of transducer placements. We have found a closed-form solution to a special case of this general problem in which it was assumed that the scatter is a weak inhomogeneity known a priori to exist within a prescribed rectangular localization domain. It was further assumed that all of the measurements are made in the far field of this domain and that each such measurement is characterized by a single temporal frequency, and a pair of incident and scattered directions and polarizations. The problem was set up in the context of statistical estimation theory with suitably defined Gaussian statistical ensembles of the scatterers and measurement errors. The criterion of optimality was chosen to be the mean square error of inversion (i.e., reconstruction of the accessible material properties of the scatterer).

${ }^{1}$ This work was sponsored by the Center for Advanced Nondestructive Evaluation, operated by the Ames Laboratory, USDOE, for the Defense Advanced Research Projects Agency, and the Air Force Wright Aeronautical Laboratories/Materials Laboratory under Contract W-7405-ENG-82.

G-8 Hardware Equipment for Real-Time Determination of Data Gained from A-Scans in Automatic Ultrasonic Inspection for Flaw Reconstruction by ALOK,R. K. NEUMANN, O. A. BARBIAN, W. BIEHL, W. SCHUHMACHER, and M. J. TAMMER, Fraunhofer-Institut für zerstörungsfreie Prüfverfahren, Univ., G. 37, D-6600 Saarbrücken, FRG.

All over the world there exists an increasing demand for more quality assurance of industrial plants, components, and especially nuclear reactors. The application of ultrasonic inspection for NDE is state of the art. A problem is still the detection of meaningful data and the reconstruction of flaws for the determination of critical defects in materials. In our institute we have developed a new kind of instrumentation. It permits flaw detection and reconstruction with more sensitivity, resolution, and accuracy by the ALOK method. Features of our hardware equipment are 1) processing of the complete $A$-scan of a transducer in real-time, 2) processing of RF signals within a dynamic range of $80 \mathrm{~dB}$ (logarithmic amplitude-compression), 3) application of an alterable algorithm for the discrimination of echo signals (maximum swing in the envelope) against noise or signal interference with high resolution of amplitude and time of flight, 4) partitioned system for use in severe environmental conditions (heat, radiation, moist, underwater), 5) application of microcomputers for easy setup, system-control, and data exchange, and 6) application of fiber optics for data trans- mission. We describe the block diagram, the function of the different units, and the processing algorithm. Furthermore we discuss our experience with this equipment in field applications.

\section{SAW TECHNOLOGY}

\section{Poster Session}

H-1 Crystal Cracking in Elastic Horn Convolvers: A Solution and its Effects on Convolver Uniformity, J. DAY and J. CALLERAME, Raytheon Research Division, Waltham, MA 02254 ; and D. L. LEE, University of Maine, Orono, ME 04469.

An important measure of performance for a wide bandwidth SAW convolver is its ability to produce an output signal whose amplitude does not depend strongly on the overlap position of two convolving signals. However, since the convolver plate on which the output is generated is actually a short electromagnetic transmission line, electrical reflections at its ends typically do give rise to an output dependent upon where the interaction occurs. In this paper we present a new simplified mathematical formulation, based on symmetry arguments, which includes transmission line losses and allows relatively easy computation of the effects of various terminations on output uniformity for a center-tapped convolver. Comparison of theory with experiment is shown. We show how this formulation explains the effect on convolver uniformity of a particularly simple solution to a substrate-cracking problem observed in elastic horn convolvers. These cracks, which typically occurred at horn-waveguide interface, had resulted in unacceptably high self-convolution levels. We also show, both theoretically and experimentally, that the convolver uniformity for a single center-tapped device is unaffected by our solution to the cracking problem. Relevance of the solution to more sophisticated convolver designs will be discussed.

H-2 Monolithic Thin Film Acoustoelectric Convolver, ${ }^{1}$ Z. S. WU, S. ONISHI, K. S. CHEN, and W.-C. WANG, Polytechnic Institute of New York, Route 110, Farmingdale, NY 11735.

Two types of configurations have been pursued for the fabrication of a thin film acoustoelectric convolver, semiconductor on $\mathrm{LiNbO}_{3}$. In one type, the output terminal plate is in direct ohmic contact with the top of the semiconductor surface, and in the other an insulating dielectric layer is inserted between the semiconductor surface and the convolver output plate. Therefore, the former has a short-circuit condition and the latter, an open-circuit condition at the semiconductor surface. Since the boundary conditions are different, the velocity, attenuation constant, and convolver efficiency are found quite different in the two configurations. Calculations on the dispersion, attenuation, and efficiency will be reported as a function of film thickness, conductivity, and frequency. In experiment, the performance of this type monolithic $\mathrm{AE}$ convolver is to be compared with the separated medium type in efficiency, dispersion, 2nd harmonic generation (due to a single SAW), and 4 th harmonic convolution signal generation.

${ }^{1}$ This work was supported in part by the Joint Services Electronics Program.

H-3 Surface Cracking on Lithium Niobate SAW Convolvers: Ferroelectric Domain Contributions, K. L. WEIL, M. K. ROY, and R. F. STEINBERG, Bendix Communications Division, Baltimore, MD 21204.

Lithium niobate $\left(\mathrm{LiNbO}_{3}\right)$, because of its high electromechanical coupling coefficient, is the most important substrate used in SAW devices. In this paper we present the evidence of aligned surface cracks, appearing on alternating edges of the metalized patterns on $\mathrm{LiNbO}_{3} \mathrm{SAW}$ convolvers, after the metalization process is completed. The reflection of SAW from these cracks introduces spurious signals, 
thereby limiting the usable dynamic range of the convolvers. Experimental results are presented suggesting that the cracking mechanism is initiated by a build-up of surface stress resulting from the creation of ferroelectric domains and internal electric fields introduced into the crystal during growth. A processing technique for eliminating these cracks will be discussed.

\section{H.4 Power Spectrum Estimation Using SAW Convolver and Chirp} Transform, ${ }^{1}$ D. SHKLARSKY and P. DAS, Electrical, Computer, and Systems Engineering Department, Rensselaer Polytechnic Institute, Troy, NY 12181.

Properties of SAW transform devices, namely SAW convolvers and SAW chirp transformer, are reviewed with respect to their capability to perform various functions in Power Spectrum Estimation systems. Usually analog power spectrum estimation is considered a delicate problem since the variance of an unbiased estimator diverges when the observation time is increased. In order to achieve reasonable estimation, a "smoothing" process should follow the estimators. Both functions, estimation, and smoothing, can be carried out by SAW devices. Two ways in which SAW signal processing elements can be used for Power Spectrum Estimation will be described. In the first, the output of a chirp SAW real time Fourier transformer is used to generate the so called periodogram estimator. In the second, a SAW convolver is used to generate the estimation. The second way is found to be very convenient to perform Cross Power Spectrum Estimation as well as Auto Power Spectrum Estimation. Finally, SAW devices, band pass filters and convolvers will be shown to perform various "smoothing" operations in order to complete an "all SAW power spectrum estimation" system.

' This work was supported in part by ARO Contract DAAG 29-81K-0066.

H-5 A Programmable Filter on GaAs, R. T. WEBSTER, Electromagnetic Sciences Division, Rome Air Development Center, Hanscom AFB, MA 01731.

An electronically programmable bandpass filter with passband nulling capability is described. The filter is implemented using a monolithic GaAs tapped delay line which mixes the input signal with a local oscillator signal in an array of FET taps. ${ }^{1}$ The output of the 32 biphase taps is programmable over a $50 \mathrm{~dB}$ dynamic range. Experimental results demonstrate bandwidths variable between $0.3 \mathrm{MHz}$ and $9.5 \mathrm{MHz}$. The passband center frequency can be varied over a $5 \mathrm{MHz}$ range. An 18 $\mathrm{dB}$ passband null can be placed in the passband. Parameters which determine the accuracy of the filter are discussed. The monolithic structure of this device would allow integration of tap control circuitry on the same GaAs substrate.

${ }^{1}$ See "GaAs monolithic SAW devices for signal processing and frequency control," T. W. Grudkowski, G. K. Montress, M. Gilden, and J. F. Black, in 1980 Ultrasonics Symp. Proc., p. 88.

H.6 DC Acoustoelectric Current Induced in a Semiconductor Film Adjacent to a SAW Substrate, ${ }^{\prime}$ K. S. CHEN, Z. S. WU, H. SCHACHTER, and W.-C. WANG, Microwave Research Institute, Polytechnic Institute of New York, Route 110, Farmingdale, NY 11735.

We have carried out a two-dimensional analysis on the dc acoustoelectric (AE) effect, both the transverse and the tangential, in a structure of semiconductor on a SAW substrate. The validity of Weinreich's relationship in such a system will be examined. The conditions under which it applies will be established. The analysis reveals that 1) the field associated with the transverse $\mathrm{AE}$ current in general tends to accumulate the semiconductor surface, 2) the polarity of the transverse AE current will, in general, not change sign when the film thickness is larger than the SAW wavelength, and 3) the diffusion effect can not be ignored in the calculation of tangential AE current. The AE current as a function of semiconductor film thickness will also be presented. Experimental verification will be provided.

${ }^{1}$ This work was supported in part by the Joint Services Electronics Program.

H-7 Nondestructive Evaluation of GaAs by AEV Measurements, ${ }^{1} \mathrm{~K}$. VARAHRAMYAN and P. DAS, Electrical, Computer, and Systems Engineering Department, Rensselaer Poly technic Institute, Troy, NY 12181.

The SAW generated acoustoelectric voltage (AEV) is used in nondestructive evaluation of the electrical surface properties of GaAs. The AEV parameters measured include polarity, peak value, and characteristic time constants. These are detected both during the SAW excitation period and soon after its termination. Measurements have been conducted on a variety of $\mathrm{GaAs}$ including high resistivity $\mathrm{Cr}$-doped and differently treated ion-implanted samples. AEV peak and time constant spectra have been obtained, some with the added effects of temperature and/or a second monochromatic light bias. This added bias condition is used to enhance and better differentiate some of the effects, such as the determination of energy levels within the bandgap and their characteristics. Considerable changes occur in both the AEV peak and time constant spectra when samples with different properties are tested. Analysis of these data based on theoretical considerations is carried out. The results have led to the determination of deep levels, surface states, and trapping behavior for high resistivity $\mathrm{Cr}$-doped samples, as well as surface carrier type, impurity activation, and annealing characteristics for ion implanted samples.

${ }^{1}$ This work was supported in part by AFOSR Grant 77-3426.

H-8 Lamb Wave Stiffening in Piezoelectric Ceramic Plates, M. PAPPALARDO, Istituto di Acustica Corbino CNR, 00189 Roma, Italy.

The electric field coupled to an elastic wave propagating in a piezoelectric media causes on appreciable variation of the elastic constant of the material. In a preceding work ${ }^{1}$ and in the Symposium in Boston last year ${ }^{2}$ we made an experimental analysis of the possibility of short circuiting this field by means of a simple electrode configuration in order to perform a temporal and spatial control of the velocity of the elastic wave. The purpose was to realize a device such as a variable delay line and an acoustic lens with variable refractive index. Both these works refer to the zeroth order symmetric Lamb waves-propagating in PZT5A piezoelectric ceramic plates. In the present work different electrode configurations are considered for the same elastic wave and material. In contrast to Raileigh waves, Lamb waves give access to two free surfaces. In this situation we can design electrode configurations which not only are able to short the tangential component of the electric field at the two free surfaces but also the normal one.

\footnotetext{
'M. Pappalardo, Appl. Phys. Lett., vol. 37, p. 10, 1980.

${ }^{2}$ M. Pappalardo, Ultrasonic Symp., 1980, Boston.
}

H-9 High Speed Data Buffering Using a $N \times M$ SAW RAM, G. F. MANES, D. GERLI, P. TORTOLI, and C. ATZENI, Istituto di Elettronica, Università di Firenze, IROE, Consiglio Nazionale delle Ricerche, Florence, 50100, Italy.

Paralleling is a standard approach when data processing is required at rates exceeding the capabilities of available digital processors. Once data have been distributed among $M$ parallel channels, they can be conveniently $\mathrm{A} / \mathrm{D}$ converted and manipulated at rates $1 / M$ lower than the original one, taking advantage of the intrinsic flexibility of digital systems. At the interface between high- and low-data rate units, however, some critical requirements do exist for analog multiplexing and Sample and Hold. A SAW random-access-memory (RAM) is described, 
arranged in a $N \times M$ configuration and based on a recent development ${ }^{1}$ of a previously discussed technique, capable of directly interfacing high rate $F$, input data to $M$ parallel channels, in form of time expanded segments. As all the buffering process is performed at rates $F / N$ or $F / M$, while the output rate is $F / M$, any critical interfacing requirement related to the input rate $F$ is avoided, thus resulting in a unique configuration for high speed data buffering.

${ }^{1}$ Electron. Lett., vol 17, no. 2, pp. 58-59, Jan. 1981.

H-10 A Surface Acoustic Wave Gas Detector, ${ }^{1}$ A. BRYANT, D. L. LEE, and J. F. VETELINO, Department of Electrical Engineering and Laboratory for Surface Science and Technology, University of Maine, Orono, ME 04469.

A dual path surface acoustic wave (SAW) delay line oscillator configuration has been fabricated to detect toxic and nontoxic gases. One delay line is coated with an organic film which selectively sorbs a particular gas while the other is uncoated. Gas absorbed by the film alters the SAW characteristics which in turn causes the oscillating frequency to change. The relative change in the frequency of the two oscillators is monitored to measure the concentration of the gas. Both $\mathrm{LiNbO}_{3}$ and quartz oscillators have been examined. Results relating to a triethonalamine film, which selectively sorbs $\mathrm{SO}_{2}$, have shown that detection of less than 70 parts per billion is possible. Discussion and results relative to device sensitivity, selectivity and reproducibility are also presented. The present gas detector offers the possibility of realizing a small, cheap, reliable and accurate commercial or military gas detector.

${ }^{1}$ This work was supported in part by NSF Grant SPI-8026415.

†H-11 Spot and Line Electron Beam Detection of Surface Acoustic Waves on $\boldsymbol{Y}-\boldsymbol{X}$ Quartz, ${ }^{1}$ N. R. ZUEF LE, Northwestern University, Department of Electrical Engineering and Computer Science, Evanston, IL 60201.

A mathematical model is presented in one dimension, along the axis of SAW propagation, for the equilibrium interaction of a stationary electron beam of diameter $D$, and a traveling SAW pulse of wave-length $\lambda$. The analysis explains the appearance for $D / \lambda>1$ of the rectified pulse envelope reported earlier ${ }^{2}$ and further indicates the carrier frequency readout for $D / \lambda<1$. Confirmation of these modulated secondary emission output signals are reported using a $20 \mu \mathrm{s}, 6 \mathrm{MHz}, Y-X$ quartz delay line irradiated with a beam energy of $<2300 \mathrm{eV}$. The 4 $\mu$ long, $1 \mathrm{KHz}$ rep-rate pulses were detected by a biased metal collector and by an electron multiplier tube. By varying the beam size and switching between the stationary spot and the forward or reverse line scan modes, variable delay, time-reversal, envelope detection, and variable pulse compression and expansion are demonstrated. Device application variables and limitations are discussed.

${ }^{1}$ This work was supported in part by the Northwestern University's Materials Research Center.

${ }^{2}$ Proc. IEEE, vol. 58, no. 2, pp. 253-254, Feb. 1970.

\section{NEW TEMPERATURE COMPENSATED PIEZOELECTRICS}

\section{Poster Session}

I-1 Investigation of Temperature Stable SBAW in Berlinite, ${ }^{1} \mathrm{~K} . \mathrm{F}$. LAU, R. B. STOKES, and K. H. YEN, TRW Electronics and Defense, Redondo Beach, CA 90278; and B.H.T. CHAl, Allied Chemical Corporation, Morristown, NJ 07960.

This paper examines the shallow bulk acoustic wave (SBAW) properties in singly rotated $Y$-cut berlinite. The rotation angles of the substrates lie in two regions near $+40.5^{\circ}$ and $-64^{\circ}$. The substrates were grown hydrothermally by the transport method. The temperature behavior of SBAW in berlinite was found to be different from that of quartz. In quartz, the frequency-temperature curve exhibits a cubic behavior for substrates with $\theta$ equal to $-50.5^{\circ}$. In berlinite, cubic behavior was found in the $+40.5^{\circ}$ substrate. The SBAW in $+40.5^{\circ}$ berlinite therefore combines good temperature stability with high wave velocity. Substrates with three different $\theta$ 's in each of the two temperature stable regions were characterized in terms of wave velocity, coupling coefficient and temperature stability. Surface acoustic wave devices were also fabricated on the same substrates to provide additional information on the substrate quality. The result showed that for limited temperature range $\left(15^{\circ} \mathrm{C}\right)$, the temperature stability of SBAW in berlinite can be comparable to that of SAW in ST quartz.

${ }^{1}$ This work was partially supported by the Army Research Office under Contract No. DAAG29-78-C-0043.

I-2 Second-Order Temperature Coefficients of Singly and Doubly Cuts of Berlinite, P. H. CARR and J. H. SILVA, RADC Electromagnetic Sciecnes Division, Hanscom AFB, MA 01731, and B.H.T. CHAI, Allied Chemical Corporation, Box 1021R, Morristown, NJ 07960.

The $80.4 X$-propagating orientation of berlinite $\mathrm{AlPO}_{4}$ has the advantage of being temperature compensated with two to four times the piezoelectric coupling of ST quartz. However, the measured secondorder temperature coefficient of $264 \times 10^{9} / \mathrm{C}^{2}$ is eight to nine times larger than ST quartz. This paper will present experimental measurements on three additional single rotated cuts and two doubly rotated cuts of berlinite whose linear temperature coefficient is predicted to be zero. ${ }^{1}$ Particular emphasis will be focused on searching for a smaller second-order temperature coefficient of importance for oscillator and filter applications.

${ }^{1}$ R. M. O'Connell and P. H. Carr, IEEE Trans. Sonics Ultrason., vol. SU-24, p. 376, 1977.

I-3 An Experimental Study of the SAW Properties of Several Berlinite Samples, ${ }^{1}$ D. BAILEY, D. L. LEE, J. ANDLE, and J. F. VETELINO, Department of Electrical Engineering and Laboratory for Surface Science and Technology, University of Maine, Orono, ME 04469 , and B.H.T. CHAl, Allied Chemical, Corporate Headquarters, Morristown, NJ 07960 .

An experimental study of the surface acoustic wave (SAW) properties for various propagation directions in four singly and two doubly rotated cuts in berlinite has been performed. The cuts studied include the $X$-axis boule $46.6^{\circ}$, the $X$-axis boule $80.4^{\circ}$, the $X$-cut $19.1^{\circ}$, the $Z$ axis cylinder $18.5^{\circ}$, and the doubly rotated $A$ and $B$ cuts. ${ }^{2}$ The SAW properties measured included the velocity, the electromagnetic to acoustic coupling and the frequency-temperature characteristics. Conclusions are made as to the suitability of any of these orientations for temperature stable device applications. Theoretical calculations were also performed and statements relative to the accuracy of the existing elastic, piezoelectric, and dielectric constants are presented.

\footnotetext{
${ }^{1}$ Work was supported by Allied Chemical Corporation.

2 R. M. O'Connell and P. H. Carr, IEEE Trans. Sonics Ultrason., vol. SU-24, p. 376, 1977.
}

I-4 Dielectric and Piezoelectric Properties of Aluminum Phosphate, E. D. KOLB. A. M. GLASS, R. L. ROSENBERG, and R. A. LAUDISE, Bell Laboratories, Murray Hill, NJ 07974, and Holmdel, NJ 07733.

Aluminum phosphate is an important piezoelectric material for SAW and other devices because of its large coupling constant and nearly temperature independent resonance frequency. Careful measurements of dielectric constant are lacking as are direct measurements of piezoelectric coupling constants. We have directly measured the dielectric constant and found a value of $5.97 \pm .05$ within experimental error, 
independent of frequency and in agreement with Mason's ${ }^{1}$ resonanceantiresonance value. We have used a novel loading technique ${ }^{2}$ to directly measure the piezoelectric coupling constants. This technique is particularly suited for very small specimens and for mapping homogeneity. We found that $d_{11}$ is $3.52 \pm 0.25 \times 10^{-12} \mathrm{CN}^{-1}$ within experimental error in agreement with Mason. ${ }^{1}$ The implications of these results for device behavior and the importance of using direct measurements of basic properties in conjunction with device studies will be discussed.

${ }^{1}$ W. P. Mason, Piezoelectric Crystals and Their Applications to Ultrasonics. New York: Van Nostrand, 1950, p. 84.

${ }^{2}$ S. C. Abrahams and J. L. Bernstein, J. Appl. Cryst., vol. 12, p. 425 , 1979.

I-5 Lithium Tetraborate-A New Temperature Compensated Piezoelectric Substrate Material for Surface Acoustic Wave Devices, ${ }^{1}$ N. M. SHORROCKS, R. W. WHATMORE, and F. W. AINGER, Plessey Research (Caswell) Limited, Allen Clark Research Centre, Caswell, Towcester, Northants. NN12 8EQ, England, and I. M. YOUNG, R.S.R.E., St. Andrews Road, Malver, Worcs. WR 14 3PS, England

Bulk wave and surface wave device measurements on lithium tetraborate single crystals have indicated that the material shows considerable promise for SAW substrates. Measurements on an $X$-cut, $Z$ propagating SAW device have shown a parabolic time delay/temperature behavior with the minimum at $12^{\circ} \mathrm{C}$, combined with a SAW coupling factor of 1.04 percent (approximately 10 times larger than ST quartz) and a SAW velocity of $3500 \mathrm{~ms}^{-1}$. The substrate shows an overall variation in time delay of $\pm 60 \mathrm{ppm}$ from 0 to $40^{\circ} \mathrm{C}$ which, while being higher than ST quartz over the same temperature range, is considerably smaller than $\mathrm{YZ} \mathrm{LiNbO}_{3}( \pm 1880 \mathrm{ppm})$. This good temperature stability, combined with the strong SAW coupling, means that it is an ideal candidate for temperature-stable SAW filters with bandwidths in the 2 to 5 percent range.

${ }^{1}$ This work was supported by D.C.V.D., Procurement Executive, Ministry of Defence, Empress State Building, London, SW6 1TR, England.

I-6 Power Flow Angle and Pressure Dependence of Saw Propagation Characteristics in Quartz, A. BALLATO and T. LUKASZEK, US Army ERADCOM, Ft. Monmouth, NJ 07703 , and D. F. WILLIAMS and F. Y. CHO, Motorola, GED, Scottsdale, AZ 85252 .

Experimental measurements of the temperature variation of the surface acoustic wave power flow angle (PFA) are shown to be in close agreement with the calculated variation. The effect of the temperature dependence of the power flow angle and the anisotropy parameter $\gamma$ on surface wave filters, reflective array devices, and delay lines are discussed both for doubly rotated and the more popular singly rotated orientations. Techniques for utilizing the temperature dependence of the surface acoustic wave power flow angle to produce temperature stable delay lines are presented. Choice of optimum doubly rotated cuts of quartz for this purpose is discussed, and experimental results are presented. Several of these orientations with promising temperature coefficients are fabricated into circular wafers. In-plane diametric forces are applied to these wafers at different azimuthal angles. Frequency shifts as a function of applied forces are measured, and compared with previous results in ST-cut quartz.

\section{SAW RESONATORS AND OSCILLATORS}

\section{Chairperson: G. K. MONTRESS}

J-1 Split Open Metal Strip Arrays and Their Application to Resonators, T. KOJIMA and J. TOMINAGA, Faculty of Engineering, Tamagawa University, Machida, Tokyo 194, Japan.
The main effects which yield the reflection phenomena for open metal strip array (OMSA) are regenerated wave (RW) and mass/electrical loading (MEL), and they counteract each other. ${ }^{1}$ For the substrates of large coupling factors $k^{2}$ such as $\mathrm{LiNbO}_{3}$, the effect of $\mathrm{RW}$ is larger than that of MEL. So, we split each strip composing array to suppress MEL by the same principles as the double electrode and obtained efficient reflectors. We call this new type of reflector "split open metal strip array" (S-OMSA) and derived its reflection coefficient in closed form by applying the image parameter theory to equivalent circuit model. The advantages of the S-OMSA are 1) its higher reflectivity per unit element and 2) its wider stop band, in comparison with conventional OMSA. Experimental data made for S-OMSA on $\mathrm{LiNbO}_{3}$ (128 rot. $Y, X$-prop.) are presented and compared with OMSA. Finally, the low spurious resonance characteristics of one- and two-port resonators utilizing these S-OMSA's are shown.

${ }^{1}$ T. Kojima, J. Tominaga, and T. Suzuki, JJAP Supplement 20-3, 1981.

J-2 SH Surface Wave Propagation on Corrugated Surfaces of Rotated $Y$-Cut Quartz and Berlinite Crystals, A. RENARD and J. HENAFF, CNET PAB, Department MAE, 196 rue de Paris, 92220, Bagneux, France, and B. A. AULD, Stanford University, Stanford, CA 94305

An analy sis has been performed of $X$-polarized horizontal shear surface wave propagation on rotated $Y$-cut quart $Z$ and berlinite corrugated surfaces. Space/harmonic analysis is used, with the effect of the corrugated surface grating modeled by Datta-Huntsinger perturbation theory. Dispersion curves are given, showing the influence of crystal orientation, grating depth, and sample thickness. Scattering coefficients for a finite length stop-band grating in an infinite pass-band grating line are evaluated using a modification of the Kogelnik-Schmidt theory for finite SAW gratings. Experimental results are presented and the potential of these structures for surface wave resonator applications is discussed.

J-3 Sensitivity of SAW Delay Lines and Resonators to Vibration, T. E. PARKER and J. CALLERAME, Raytheon Research Division, Waltham, MA 02254.

One of the major advantages of SAW controlled oscillators is their very low level FM noise sidebands. However, in many applications where this low noise level is important, the oscillator must operate in an environment with high vibration levels. Evaluating the degradation of noise sidebands due to vibration is very difficult at this time since very little information exists in the literature. Furthermore, the information that is available was obtained from measurements in which the entire oscillator was subjected to vibration rather than just the SAW device. This greatly complicates the situation since mechanical resonances may be present in the circuit board. Static $g$-force sensitivity measurements eliminate the problem with resonances, but the published results have been inconsistent with the vibration measurements. Therefore, to evaluate the inherent sensitivity to vibration of rotated $Y$-cut quartz SAW devices, a number of measurements have been made in which only the SAW device was subjected to vibration. A number of different substrate-mounting techniques have been evaluated for both delay lines and resonators. The devices were vibrated in each of three perpendicular directions with both random and sinusoidal vibration used. The observed sensitivity to vibration varied by two orders of magnitude for different mounting geometries, with the lowest sensitivities being comparable to that of a well-designed AT-cut bulk wave resonator.

J-4 A Digitally Compensated SAW Oscillator, A. J. SLOBODNIK, Jr., R. D. COLVIN, G. A. ROBERTS, and J. H. SILVA, Rome Air Development Center, Hanscom Air Force Base, MA 01731. 
A method of using simple digital circuitry to compensate for and thereby reduce the temperature sensitivity of surface acoustic wave (SAW) oscillators will be described. Initial results with an AT-cut quartz SAW oscillator show a reduction in frequency variation from $191.7 \mathrm{ppm}$ to $5.2 \mathrm{ppm}$ over the temperature range $-13^{\circ}$ to $+97^{\circ} \mathrm{C}$. Substantial further improvement is projected when sealed test packages designed for this application are used. A key feature of this digitally compensated SAW oscillator (DCSO) is the use of a novel temperature sensing scheme which minimizes thermal contact and thermal time constant problems. Additional features of the DCSO include fast warmup and low cost potential.

J-5 A Wideband Frequency Memory Using an Overmoded SAW Oscillator, C. M. BOYNE and M. F. LEWIS, Royal Signals and Radar Establishment, Malvern, Worcestershire, UK.

This paper is concerned with a frequency memory system which has an unlimited storage time. Such a system has been built, with a set-on accuracy of $\pm 5 \mathrm{MHz}$ in a bandwidth $>500 \mathrm{MHz}$, and can be triggered from a single RF input pulse of duration $100 \mathrm{nsec}$ or greater. To achieve this, two overmoded (sometimes called "multimoded") oscillators were combined. The coarser of these employed a GaAs FET and a length of coaxial cable giving a mode separation of $75 \mathrm{MHz}$ across the full operating bandwidth. The finer set-on accuracy was achieved using an overmoded SAW oscillator exhibiting 10 "clean" modes across a bandwidth essentially equal to the mode separation of the coarse overmoded oscillator. The operation of this system will be described, with emphasis on the SAW oscillator loop. Some possible applications will be discussed and potential improvements to the memory system suggested.

J-6 SAW Reflection from Aluminium Strips on $\mathrm{LiNbO}_{3}$, A. G. STOVE and R. C. WOODS, Department of Engineering Science, University of Oxford, Oxford, England.

The variation of reflection amplitude with wavelength of a surface acoustic wave incident normal to a thin single insulated metal strip on lithium niobate has been predicted. ${ }^{1}$ The predictions are anomalous in the sense that a peak in reflectivity occurs when the wavelength is twice the strip width. This paper presents amplitude and phase measurements which are in broad agreement with the theory. Observations will also be presented for the reflectivity of groups of parallel identical strips and of strips with a thin metal overlay covering the surface.

${ }^{1}$ E. L. Cambiaggio and F. C. Cuozzo, IEEE Trans. Sonics Ultrason., vol. SU-26, p. 340, 1979.

\section{MEDICAL ULTRASOUND: IMAGING}

\section{Chairperson: J. D. LARSON}

† K-1 Detailed Analysis of Physical Factors Affecting Pulse-Echo Images, D. LEE, F. S. FOSTER, M. ARDITI, and J. W. HUNT, The Ontario Cancer Institute, Toronto, Ontario, Canada M4X $1 \mathrm{~K} 9$.

An annoying property of clinical pulse-echo images is the speckled appearance of homogeneous structures. The speckle interferes with the visibility of small lesions. To study its properties in detail, $15 \mathrm{~mm} \times$ $15 \mathrm{~mm}$ images of phantoms were digitally recorded. The transducer assembly consisted of a five element, $4 \mathrm{MHz}$, electronically focused annular array axially aligned with a large aperture conical transducer. This allowed the same plane to be scanned using various focussing geometries. The RF waveform was digitized directly af ter the pre-amplifier at a sampling interval of $0.2 \mu \mathrm{s}$. Time-gain compensation, demodulation, and grey-level assignment were performed digitally. The RF image may also be displayed directly, in which dark areas correspond to troughs in the waveform, bright areas to peaks, while the grey areas represent zero signal. Phantom inages both in and out of focus, were recorded using the various focussing techniques. The speckle patterns produced by the different transducer geometries are very different. The coarseness of the spcckle is closely related to the beam profile. Direct display of the RF images suggests that speckle is partly due to phase changes as the transducer moves laterally. This suggests that improved focussing techniques and appropriate signal processing techniques may reduce speckle without loss of spatial or contrast resolution.

K-2 Sidelobe and Speckle Reduction for an Eight Sector Conical Scanner, M. S. PATTERSON and F. S. FOSTER, The Ontario Cancer Institute, Toronto, Ontario, Canada, M4X $1 \mathrm{~K} 9$.

We have developed a hybrid system for ultrasonic breast imaging which uses an annular array as a transmitter and a large conical transducer as a receiver. This device maintains good lateral resolution (FWHM $=0.3 \mathrm{~mm}$ ) over a depth of $8 \mathrm{~cm}$ and has produced excellent in vivo B-scan images. However, this hybrid system has relatively high sidelobes which cause some loss of image contrast. In addition, the B-scans show the undesired speckled appearance characteristic of coherent imaging systems. We have investigated the possibility of improving the images by radially dividing the cone into eight sectors and processing the signals from each sector individually. We will present theoretical and experimental results showing the reduction in sidelobes that can be achieved by proper phasing of the received signals from the cone sectors before summation. We will also show that speckle can be decreased by demodulating the RF signals from each sector before they are summed.

K-3 [Invited] A Comparison of Finite Aperture Phase Sensitive and Phase Insensitive Detection in the Near Field of Inhomogeneous Material, L. J. BUSSE, Bat telle-Pacific Northwest Laboratory, Richland, WA 99352, and J. G. MILLER, Washington University, Department of Physics, St. Louis, MO 63130.

An analysis is presented of the responses of finite sized phase sensitive and phase insensitive ultrasound receivers when each is used to detect spatially nonuniform pressure distributions. These nonuniform ultrasonic fields can result from a variety of wave propagation phenomena: scattering by nonrandom media, diffraction in the near field of irregular objects, transmission through inhomogeneous materials, and reflection from surfaces with texture. We present a model which allows the detection properties of phase sensitive and phase insensitive receiver apertures to be predicted easily for complex source distributions. This model is based upon the computationally efficient "backward wave" propagation technique. Experimental verification of these predictions is obtained using a piezoelectric and acoustoelectric receiver as phase sensitive and phase insensitive receivers, respectively. Results for transmission measurements are presented as frequency dependent attanuation determinations. For scattering measurements results are presented as the observed angular and spatial dependence of scattering from a simple source distribution. This study contrasts the different detection properties of phase sensitive and phase insensitive receivers and shows that the inherent properties of phase insensitive receivers can be used to advantage in many practical measurement situations.

K-4 Simultaneous Multifrequency Ul trasonography; The Principle and Technology, H. MIWA, H. HAYASHI, and T. SHIMURA, Fujitsu Laboratories Inc., Kawasaki, 211, Japan.

Slow velocity of sound limits scan depth times scan line number per unit time. Poor image of echography, prohibition of simultaneous multifunctional application, etc., are due to this barrier. Simultaneous 
transmission of differently featured multipulses and discriminating each pulse by the feature are devised as the break through. Burst pulse, chirp modulation and pseudo random coding for two different frequencies are evaluated by simulation and/or experiment on crosstalk, axial resolution, time sidelobe, dynamic range, harmonics generation, etc. Real time compound echocardiograph and bicardio tomographic planes at perfect simultaneity are shown as typical applications.

K-5 Synthetic Focussing by Means of Wave-Field Extrapolation. A New Imaging Technique for Medical Ultrasound, J. RIDDER, L. F. V. D. WAL, and A. J. BERKHOUT, Group of Acoustics, Physics Department, Delft University of Technology, The Netherlands.

In this paper it is argued that in principle the lateral resolution problem should be approached from the theory of wave field extrapolation. It is shown that optimum lateral resolution properties for a reflecting discontinuity are obtained if the propagation effects from the transducer surface to the discontinuity and vice versa are removed from the echo measurements. Using wave-theory, this can be done by an inverse wave-field extrapolation operator. Hence, by inverse wave-field extrapolation the transducer plane is simulated inside the medium of investigation. We will see that this new approach to "focusing" is most flexible and will lead to results, the resolution of which approaches the theoretical ultimate. Some images obtained with this method will be shown.

\section{PHYSICAL ACOUSTICS III: APPLICATIONS}

\section{Chairperson: M. LEVY}

L-1 [Invited] Application of an Ultrasonic Focusing Radiator for Acoustic Levitation of Submillimeter Samples, ${ }^{1}$ M. C. LEE, Jet Propulsion Laboratory, California Institute of Technology, Pasadena, CA 91109.

An acoustic apparatus has been specifically developed to handle samples of submillimeter sizes in a gaseous medium. This apparatus consists of an acoustic levitation device, deployment devices for small liquid and solid samples, heat sources for sample heat treatment, acoustic alignment devices and data acquisition instrumentation. The levitation device includes a spherical aluminum dish of 12-in diameter and 0.6 -in thickness, 130 pieces of PZT transducers attached to the back side of the dish and spherical concave reflector situated in the vicinity of the center of curvature of the dish. The focusing radiator levitating device operates at 75,105 , and $163 \mathrm{kHz}$, respectively. It has been demonstrated that a sample with a specific gravity as large as 19.3 can be levitated in this apparatus. The lateral positional wandering of the sample in the force well is estimated at less than 10 percent of the dimension of the sample size used. This apparatus can be used most advantageously in the coating of a submillimeter sample and the manjpulation of a compound droplet system for fusion target applications.

${ }^{1}$ This work was supported by NASA Contract No. NAS7-100.

\section{L-2 An Automatic Method for Measuring Sound Velocity with High} Resolution, M. VON SCHICKFUS, T. BAUMANN, and R. $L$. MELCHER, ${ }^{1}$ Max-Planck-Institut für Festkörperforschung, Heisenbergstr. 1, D-7000 Stuttgart 80, West Germany.

Our method is a modification of the frequency modulation CW technique developed by R. L. Melcher et al. ${ }^{2}$ There a lock-in amplifier produces a signal proportional to the deviation of the oscillator carrier frequency from an acoustic resonance of the sample. Correction is achieved by tuning the oscillator manually. In our modification we feed the output of the lock-in amplifier through an analog integrator into the FM input of the RF oscillator to shift the center frequency towards the sample's resonance similar to an AFC circuit. The frequency is measured by a counter with high integration times to increase the resolution (better than $10^{-7}$ ). The counter is connected to a calculator for continuous automatic recording and further data processing. An important feature of this system is that the sensitivity mentioned above is obtained at very low oscillator output levels $(<-34 \mathrm{dBm})$ allowing measurements in the temperature range of a dilution refrigerator without heating the sample. For example, we could observe a very small logarithmic increase of the sound velocity $\left(\sim 4 \cdot 10^{-7} /\right.$ decade in $T$ ) in $z$-cut quartz for $0.02 \mathrm{~K}<T<5 \mathrm{~K}$. We believe that this is caused by the glassy character of the bond.

' On leave from IBM T. J. Watson Research Center, Yorktown Hts., NY 10598

${ }^{2}$ R. L. Melcher, D. I. Bolef, and J. B. Menes, Rev. Sci. Instr., vol. 39, p. $1918,1968$.

L-3 Interior Versus Exterior Creeping Waves in Acoustic Resonance Scattering, ${ }^{1}$ G. C. GAUNAURD and H. ÜBERALL, ${ }^{2}$ Naval Surface Weapons Center, White Oak, Silver Spring, MD 20910.

In acoustic scattering from elastic objects, resonance features occur in the returned echo at frequencies where the object's eigen-f requencies are located, which are explained by the excitation of interior creeping waves. Corresponding resonance terms may be split off from the total scattering amplitude, leaving behind an apparently nonresonant background amplitude. This is demonstrated for scatterers of separable geometry (e.g., spheres), and also for scatterers of arbitrary geometry, using the $T$-matrix approach. It is shown subsequently that the background amplitude can be split further into specularly reflected and geometrically transmitted contributions, plus highly attenuated resonance terms which are explained by the excitation of exterior (Franztype) creeping waves. The singularity structure of the scat tering function is shown mathematically, using the $R$-matrix approach of nuclear scattering theory, as that of a meromorphic function without any additional "entire function" (as had been postulated by the singularity expansion method).

1 This work was supported by Office of Naval Research, Code 421. DC.

2 Also at the Department of Physics, Catholic University, Washington,

L-4 Refined Determination of the Rigidity Modulus by Echo Sounder Measurement, K. G. FOOTE, Department of Applied Mathematics, University of Bergen, 5014 Bergen, Norway.

Precise knowledge of the rigidity modulus is essential to theoretical determination of the acoustic backscattering cross sections of elastic spheres used for calibrating hydroacoustic equipment. This can be obtained with high accuracy from echo sounder measurements on several spheres made of the subject material, given some prior knowledge of the elasticity. Two methods are described. Both involve choosing the diameters of the spheres with respect to their estimated backscattering properties so that the echo energy (method 1) or echo waveform (method 2) will be particularly sensitive to small changes in the rigidity modulus from its nominal value. The precise value is then determined by the exercise of theory assuming that the rigidity modulus is the single free parameter. Applications of the first method to a commercial, electrical grade copper of density $8947 \pm 2 \mathrm{~kg} / \mathrm{m}^{3}$ reveal that the rigidity modulus is $46.86 \pm 0.06 \mathrm{GPa}$.

L-5 Reconstruction of Acoustic Impedance Profile from the Reflection Impulse Response, ${ }^{\text {I }}$ P. C. PEDERSEN, O. J. TRETIAK, and P. HE, Biomedical Engineering and Science Institute, Drexel University, Philadelphia, PA 19104.

If the reflection impulse response of a nonattenuating medium with a one-dimensional continuous impedance profile is given, the acoustic 
impedance as a function of travel time can be reconstructed, provided the interrogating wave propagates in the direction of the impedance change. In this paper we first discuss two different reconstruction techniques under normal incidence. One technique, utilizing the impediography equation, considers only the first-order reflections while the other technique which takes into account all the higher order reflections as well is based on a solution by Goupillaud. The input for the reconstruction is the reflection impulse response, calculated from the given acoustic impedance profile. The computer reconstruction is carried out for a number of impedance profiles. For small impedance variations, both techniques provide accurate results while only Goupillaud's solution provides valid results for large impedance variations. In order to reconstruct both velocity and density as a function of spatial distance, the reflection impulse response must be determined for two different incident angles. We will present data for such reconstruction, using Goupillaud's solution.

${ }^{1}$ This work was supported by NSF Grant ENG78-04348.

\section{SAW RESONATOR FILTERS}

\author{
Chairperson: T. E. PARKER
}

M-1 280 MHz SAW Resonators in Production, S. ELLIOTT, M. MIERZWINSKI, and P. PLANTING, Hewlett Packard, Santa Rosa Technology Center, Santa Rosa, CA 95404.

A $280 \mathrm{MHz}$ SAW resonator was released to production in February of this year. The device was designed as a retrofit replacement for a bulk-wave crystal resonator operated at its eleventh overtone in two Spectrum Analyzer models currently manufactured by Hewlett Packard. Although the SAWR has a lower $Q$ than the bulkwave device, problems associated with close-in modes and poor field reliability of the latter are alleviated by the SAWR. The minimum unloaded $Q$ (at atomospheric pressure) of these devices is 15000 with a nominal oneport series-resonant resistance of $30 \Omega$. Several unique problems were encountered in developing designs and processes for production of SAW devices. These problems arose from the extreme frequency sensitivity of the device to processing parameters and to contamination. We have developed processes for production and packaging of SAWR devices in a standard one-port crystal package with high yield and good reliability.

M-2 Power dependence of Aging in SAW Resonators, W. R. SHREVE, Hewlett-Packard Laboratories, Palo Alto, CA 94304, and R. C. BRAY, S. ELLIOTT, and Y. C. CHU, Santa Rosa Tech Center, HewlettPackard, Santa Rosa, CA 95404.

The aging rate of SAW resonators in oscillators has been found to vary as the operating power is varied. Increasing the power can cause the aging rate to vary from a few ppm per year to more than a part per thousand per year. Tests have been run passively (no power dissipated in the resonator) and actively at levels from $-10 \mathrm{dBm}$ to $+20 \mathrm{dBm}$. The major cause of the power dependent aging is acoustically induced migration of the aluminum in the coupling transducers. ${ }^{1}$ The addition of a small amount of copper to the film was found to reduce or eliminate these migration effects and allow stable operation at high power levels. Test results, an analysis of the power density in the resonator cavity, and a detailed description of the migration phenomenon are included.

'J. 1. Latham, et al., "Thin solid films," vol. 64, pp. 9-1 5, 1979.

M-3 Multipole SAW Resonator Filters: Elements of Design, Fabrication, and Frequency Trimming, ${ }^{1}$ W. J. TANSKI, Sperry Research Center, Sudbury, MA 01776.
We are currently developing multipole $\mathrm{SAW}$ resonator filters which must have high rejection levels $(>60 \mathrm{~dB})$, low losses $(<6 \mathrm{~dB})$, filter $Q$ 's $\sim 4000$, and small shape factors $(\sim 4: 1)$ in the VHF range. In this paper we discuss the salient aspects of filter design and fabrication including a description of a new resonator frequency trimming technique which is essential in producing these devices. For filter design, we use the resonator synthesis procedures developed by Matthaei along with detailed equivalent circuit analysis calculations to establish and perfect the designs. Intercavity coupling is by transducers or reflectors, and we find that to attain $60 \mathrm{~dB}$ rejection a minimum of two transducer couplings (three cavities) are necessary. The filter design is synchronous, that is, each pole must resonate at the same frequency $\left(\omega_{0}\right)$ when coupled. Techniques we have developed for meeting this requirement in practice will be presented including a discussion of our frequency trimming procedure. In this procedure we adjust the resonant frequency of each pole by modifying only the transducer configurations (electrode step height) and utilizing changes in transducer reflectivity to shift the frequency downward. Experimental results for three and six-pole devices will be presented to demonstrate the performance attained.

${ }^{1}$ This work is supported by the U.S. Naval Research Laboratories, Contract No. N00014-81-C-2066.

M-4 SAW Resonator Filters with Optimized Transducer Rejection, T. F. O'SHEA and R. C. ROSENFELD, Sawtek Inc., 2541 Shader Rd., Orlando, FL 32854.

SAW resonator filters achieve low-loss narrow-band performance with moderate close-in rejection. The close-in rejection is limited by the relative broad-band interdigital transducers. The transducers provide additional rejection at wider bandwidths, but they are usually weighted for transverse-mode suppression and, therefore, do not provide optimum rejection. This paper presents $S A W$ resonator filters in which the transducers are optimized for high rejection. Two-pole filters are demonstrated at $150 \mathrm{MHz}$ with $2 \mathrm{~dB}$ insertion loss, $70 \mathrm{KHz}$ $1 \mathrm{~dB}$ bandwidth, and $70 \mathrm{~dB}$ rejection at $\pm 4 \mathrm{MHz}$. Similar four-pole filters achieve $4 \mathrm{~dB}$ insertion loss and greater than $100 \mathrm{~dB}$ rejection at $\pm 4 \mathrm{MHz}$. Filter results at UHF frequencies and design procedures are also presented.

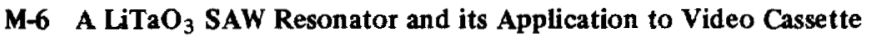
Recorder, Y. EBATA, K. SATO, and S. MORISHITA, Toshiba Research and Development Center, Kawasaki, 210, Japan.

A $100 \mathrm{MHz} \mathrm{LiTaO}{ }_{3} \mathrm{SAW}$ resonator has been developed. It was mass produced and successfully used as a local oscillator in a video cassette recorder (VCR) RF convertor. This new resonator, constructed on $\mathrm{LiTaO}_{3}$ substrate (X-cut, $112^{\circ} \mathrm{Y}$ ) for the first time, with 15000 unloaded $Q$ was fabricated within a $\pm 30 \mathrm{KHz}$ resonance frequency deviation by large scale batch processing. Aluminum strips were selected as reflective arrays on the substrate. The SAW reflection coefficient and the stored energy effect at the strip edge were precisely measured for various aluminum film thicknesses. As a result, the aluminum reflector on $\mathrm{LiTaO}_{3}$ proved excellent from viewpoint of realizing small chip size high $Q$ resonators and good frequency reproducibility comparing with other reflector structures (grooves on quartz). An optimum design method, using the measured parameters, was developed to make oneport resonators adaptable to any oscillator circuit. Reliabilities were also tested and met specifications for VCR application. In addition, the stress-induced metal migration was investigated in detail. The migration proved to reduce the resonance frequency and the $Q$ value in electric performances, likewise to affect reflective arrays mainly but IDT electrodes very slightly. Improving the input power limitation, 0.3 percent $\mathrm{CU}$ doping in aluminum was found to be effective and to extend the limit by at least 50 times. 


\section{ACOUSTIC MICROSCOPY: TECHNIQUES}

\section{Chairperson: C. F. QUATE}

N-1 Experiments with the Planar Acoustic Microscope Lens, G. W. FARNELL and C. K. JEN, Department of Electrical Engineering, McGill University, Montreal, Quebec, Canada, H3A 2 K6.

In the planar microscope lens, circular electrodes on a piezoelectric substrate produce a converging Rayleigh wave which converts to a conical beam in the liquid. Such lenses have been mounted in a scanning acoustic microscope geometry and used at various frequencies in the hundred megahertz range to produce images with a resolution equal to the wavelength in the liquid. Using the fabrication convenience of the lens various other imaging experiments have been performed. If, for example, one of these lenses is made of two semicircles, then one semicircle can be used as the transmitter and the other as the confocal receiver in reflection imaging with relatively small direct coupling between the two. Similarly, if one of the lenses consists of two semicircles driven in phase opposition the resulting images are highly textured because of the split-beam illumination. Yet other images are formed if both lenses are made of semicircles and crossed pairs are used for transmitting and receiving. Nonlinear effects are easily produced in the liquid and the wide bandwidth of the transducers allows the excitation at one frequency and the reception at harmonic frequencies with the attendant resolution of the latter. Similarly with a lens made of two semicircles, each can be excited at a different frequency and the reception made at the sum or difference frequency. When the liquid is driven into nonlinearity, acoustic streaming of rather unusual cross section is observed in the illuminated region.

N-2 Acoustic Anisotropy Detection of Materials by Acoustic Microscope using Line-Focus Beam, J. KUSHIBIKI, A. OHKUBO, and N. CHUBACHI, Department of Electrical Engineering, Faculty of Engineering, Tohoku University, Sendai, Japan.

The anisotropy detection of acoustic properties of solid materials by means of reflection acoustic microscopes in nonscanning version is investigated. For the purpose, an acoustic line-focus beam is newly introduced as a pick-up sensor in place of usual circularly focused beams. The line-focus beam that is linearly focused along one axis is readily formed by an acoustic lens with a cylindrical concave surface. With use of the line-focus beam, acoustic anisotropies of materials can be properly detected as a function of angle around the beam axis normal to the sample target through measuring phase velocities of leaky surface acoustic waves that are dependent on the wave propagation directions, in the same procedure as observing the acoustic material signature ${ }^{1}$ with use of circularly focused beams. Experiments for sapphire and lithium niobate crystals are demonstrated by using an acoustic sapphire lens with a cylindrical concave surface of $1.0 \mathrm{~mm}$ in radius at a frequency of $200 \mathrm{MHz}$. The measured results are compared to the calculated results, and it is proved that the anisotropy of materials can be successfully detected by acoustic microscopes using the line-focus beam.

${ }^{1}$ R. D. Weglein, Appl. Phys. Lett., vol. 34, pp. 179-181, 1979.

N-3 Acoustic Microscopy: Recent Applications in Semiconducting Devices, J. ATTAL, G. CAMBON, and M. ROUZEYRE, Centre d'Etudes D'Electronique des Solides, (CNRS), Université des Sciences et Techniques du Languedoc, 34060-Montpellier-Cedex, France.

In recent works we have demonstrated that the acoustic microscope using focused lenses was able to image through or inside opaque materials. Metallic liquids such as mercury are necessary to insure the transit of acoustic energy toward the object with minimum losses. The operating frequency can be easily raised up to $2 \mathrm{GHz}$ with a conven- tional $0.2 \mathrm{~mm}$ diameter lens, corresponding to a resolution in the range 0.3 to $1 \mu \mathrm{m}$ and a penetration up to $20 \mu \mathrm{m}$. Typical applications of this high frequency microscope are reported; such as annealing amorphous silica on silicon by means of a beam laser scan. On the other hand, we present the low frequency microscope operating in the range 50 to $200 \mathrm{MHz}$ with a lens diameter around $5 \mathrm{~mm}$ which allows a penetration of about $0.5 \mathrm{~mm}$ in silicon with a lateral resolution of the order of $10 \mu \mathrm{m}$. Applications in nondestructive evaluation of bonding contacts of integrated circuits are shown.

†N-4 Resolution Improvement in the Acoustic Microscope Using High Intensity Focused Beams, ${ }^{1}$ D. RUGAR, E. L. Ginzton Lab, Stanford University, Stanford, CA 94305.

This paper describes the results of nonlinear imaging experiments using a scanning acoustic microscope operating in pulsed reflection mode. The microscope transmits and receives at the fundamental frequency $(2.0-2.8 \mathrm{GHz})$ and utilizes an acoustic lens with radius of only $18 \mu \mathrm{m}$. The small lens radius minimizes liquid path attenuation and allows intensities in excess of $10^{5} \mathrm{~W} / \mathrm{cm}^{2}$ to be achieved in the vicinity of the focus. At these intensities the propagation of sound is highly nonlinear. In particular, it is found that the resolving power of the microscope increases significantly when high power levels are used. Resolution improvement by a factor of 1.4 over the linear (low power) case is demonstrated. The onset of the resolution improvement is seen to coincide with depletion of power in the fundamental due to harmonic generation. Using this effect a grating with $0.3 \mu \mathrm{m}$ period was resolved in hot water and one with $0.2 \mu \mathrm{m}$ period was resolved in liquid nitrogen. A simple physical model is presented to explain the resolution improvement.

'This work was supported by the Office of Naval Research.

† -5 Acoustic Microscopy in Gases at $450 \mathrm{~nm}$ Wavelength, ${ }^{1} \mathrm{~F}$. FARIDIAN and H. K. WICKRAMASINGHE, Department of Electrical Engineering, University College, London, England.

It has previously been shown that by resorting to gases under pressure, it should be possible to build an acoustic microscope with suboptical resolution. Gas medium acoustic microscopy has now been developed to the point where it is possible to operate in argon at 50 bar and $700 \mathrm{MHz}$; the corresponding wavelength at room temperature is around $450 \mathrm{~nm}$. We have used an integral condenser lens to illuminate the very small objective that is required to attain such short wavelengths. The basic design of our system together with results obtained from the new (condenser/objective) lens combination will be presented.

\footnotetext{
${ }^{1}$ This work was supported by the National Research Development Corp.
}

N-6 Quantitative Comparison of Calculated and Measured Flaw Characteristics, D. E. YUHAS, M. G. ORAVECZ, and L. W. KESSLER, Sonoscan, Inc., Bensenville, IL 60106.

The first quantitative comparison between scanning laser acoustic microscope (SLAM) image data and calculations based on scattering theory are presented. Buried flaws in ceramics are first detected by direct observation of the images in real time. Data is recorded by digitization of either selected lines or the entire frame. Samples containing flaws are then destructively analyzed to verify the flaw type and size. Our results indicate that the theory qualitatively predicts several observed image characteristics. However, SLAM images of flaws in silicon nitride show less beam divergence than expected from compressional wave scattering theory. 


\section{PHYSICAL ACOUSTICS IV: PHYSICAL ACOUSTICS}

\section{Chairperson: M. A. BREAZEALE}

0-1 Attenuation of Static Polarization Echo in $\mathrm{SiO}_{2}$ Powder by ${ }^{4} \mathrm{He}$ Film, M. POIRIER, Institut de Génie des Matériaux, C.N.R.C., Montréal, Québec, Canada, and J.D.N. CHEEKE, Dép. de physique, Université de Sherbrooke, Sherbrooke, Québec, Canada J1K 2R1.

Dynamic and static polarization echoes have been observed in many piezoelectric powders. The "memory" echo which results from the formation of a static internal polarization of individual particles following the application of a two RF pulse sequence is quite stable and has a long decay constant $T_{1}$ (> lifetime of any reasonable dynamic process). If ${ }^{4} \mathrm{He}$ gas is surrounding the powder, two factors will produce an attenuation of the phonon echo as well as the memory echo, the acoustic impedance mismatch to the gas and also the adsorbed helium film. In our experiments, the memory echo will serve as a test to study ${ }^{4} \mathrm{He}$ film on $\mathrm{SiO}_{2}$ powder $(63<\phi<75 \mu \mathrm{m})$ which is inserted between the plates of a capacitor cell to be submitted to an RF electric field at $35 \mathrm{MHz}$. We report the attenuation curves of the memory echo as a function of the ${ }^{4} \mathrm{He}$ pressure at different temperatures of the liquid helium bath. The obtained attenuation curves are interpreted with the aid of the adsorption isotherms of the powder.

O-2 Magnetic Field Effects on Plate Elastic Wave Propagation in Metallic-Glass Ribbon, M. POIRIER, Institut de Génie des Matériaux, C.N.R.C., Montréal, Québec, Canada; L. GERMAIN and J.D.N. CHEEKE, Dép. de physique, Université de Sherbrooke, Sherbrooke, Québec, Canada J1K 2R1.

Elastic waves (few $\mathrm{MHz}$ ) were generated and detected in a metallic glass ribbon $\mathrm{Fe}_{81} \mathrm{~B}_{13,5} \mathrm{Si}_{3,5} \mathrm{C}_{2}$ using bonded piezoelectric transducers. Tow types of plate wave were propagated and studied in a plane oriented dc magnetic field: the lowest symmetrical and non-dispersive SH mode and the zeroth symmetrical Lamb wave which presents only little dispersion as $\omega d \rightarrow 0$. Wave amplitude is found to be strongly magnetic field dependent for the two modes in the saturation regime; it increases up to a field saturation value of a few hundreds of oersteds and field orientation effects are important. Velocity variation is positive for increasing field intensity with a saturation value decreasing with frequency. Those iron-rich amorphous ribbons are magnetostrictive and they show a very large magnetoelastic coupling via a giant $\Delta E$ effect. A tentative explanation of the results is presented.

0-3 The Molecular and Macroscopic Anisotropy of $\mathbf{P V F}_{2}$, E. BALIZER and S. T. KOWEL, Department of Electrical and Computer Engineering, Syracuse University, Syracuse, NY 13210.

The elastic and loss constants of $\mathrm{PVF}_{2}$ films have previously been characterized by ultrasonic measurements according to a $2 \mathrm{~mm}$ symmetry. However, these films are semicrystalline with the crystalline content having three polymorphs, the $\alpha, \beta$, and $\gamma$ phases. Measurements of the orientation distribution function for these phases were taken by infrared dichroism. ${ }^{1}$ The absorption bands indicate that the crystalline phases are not oriented along the preferred directions of the film as is normally assumed for ultrasonics applications. The anisotropy found by IR at the molecular level is compared with the macroscopic elastic anisotropy as found by acoustic measurements. The results reveal the correct macroscopic symmetry and are indicative of the sensitivity of the ultrasonic measurements to the crystalline phase anisotropy of the $\mathrm{PVF}_{2}$ film.

\footnotetext{
${ }^{1}$ W. M. Prest, Jr. and D. J. Luca, Soc. Plast. Eng. Tech. Paper, vol. 23 , p. $376,1977$.
}

†0-4 High-Field Losses of Polyvinylidene Fluoride Transducers, ${ }^{1}$ W. H. CHEN and C. Y. CHEN, Department of Physics, Chung Cheng
Institute of Technology, Ta-Hsi, Tao-Yuan, Taiwan 335, Republic of China.

Electrical, acoustical, and thermal performance of polyvinylidene fluoride $\left(\mathrm{PVF}_{2}\right)$ transducer has been theoretically and experimentally studied. A general theory, based on the impedance matrix model, for predicting the performance of an acoustic transducer made from a piezoelectric material with nonnegligible dielectric loss and mechanical loss has been formulated and represented using an equivalent circuit. Computer programs were designed to predict the electrical input impedance as a function of frequency of loaded $\mathrm{PVF}_{2}$ transducers, to calculate the insertion loss and bandwidth performance of the transducer. These programs were also used to model the radiated acoustic power as a function of input electric field, and the temperature rise of the $\mathrm{PVF}_{2}$ film under high electric field, as high as $30 \mathrm{v} / \mu \mathrm{m}$, operation. The dielectric and acoustic losses, and dielectric constant in oriented $\mathrm{PVF}_{2}$ films were measured from $0.5 \mathrm{MHz}$ to $100 \mathrm{MHz}$. The measured loss tangent, $\tan \delta_{D}$ and $\tan \delta_{M}$ as a function of frequency, and $\epsilon(\omega)$ were used to model the dielectric and acoustic losses in the theoretical calculation. Temperature rise as a function of applied field and frequency, and the efficiency of the transducer are predicted and compared with that of PZT transducer.

${ }^{1}$ This work was supported by the National Science Council of R.O.C.

0-5 A Study of Ultrasonic Injection Technique for Measurement of Physical Parameters in CdS and $\mathrm{As}_{2} \mathrm{~S}_{5},{ }^{1} \mathrm{Y}$. TOKUNAGA, Kanazawa Technical College, Nonoichi, Ishikawa 921 and T. HATA and T. HADA, Kanazawa University, 2-40-20, Kotatsuno, Kanazawa 920, Nippon.

Acoustic domain has been used in the past to determine the lattice attenuation factor $\alpha_{l}$ and electronic loss factor $\alpha_{0}$ in CdS, ZnO, and GaAs. However, a measurement of $\alpha_{l}$ and $\alpha_{0}$ by the dual-pulse method using the acoustic domain poses a problem one should not ignore. In this paper, we describe the ultrasonic injection technique (UIT) as a better technique than the dual-pulse method to measure the $\alpha_{l}$ and $\alpha_{0}$. Obtainable results within $0.4 \mathrm{GHz}$ and $2 \mathrm{GHz}$ using UIT are described as: 1) $\alpha_{l}$ is proportional to $f$-square ( $f$ : frequency). 2) experimental $\alpha_{0}$ agrees qualitatively with the theoretical values obtained from Hutson-White theory. 3) Average Grüneisen parameter is 0.33 . Also discussed are: 1) absorption coefficient with $200 \mathrm{MHz}$ to $400 \mathrm{MHz}$ of amorphous $\mathrm{As}_{2} \mathrm{~S}_{5}$ measured by UIT and 2) application of strong shock excited wave amplified by UIT.

${ }^{1}$ The work reported in this paper was partially supported by 1980 Grant-in-Aid for Scientific research from the Ministry of Education in Japan.

†0-6 Ultrasonic Detection of Free Radicals in Polymers, M. MONGY, A. AZIZ, National Institute for Standards, Tahrir Street, Dokki-Cairo, Egypt, and M. EL-SABEE, Chemistry Department, Cairo University, Egypt.

Measurement of the change of ultrasonic absorption as a function of time in irradiated polymers was found to detect the formation and decay of free radicals in polymers. The phenomena could be detected in amorphous polymers which exhibit degradation by irradiation such as PMMA, PEMA, and Nylon 6 and those which exhibit cross linking by irradiation such as PE. The energy required for the decay of free radicals, which are formed due to irradiating the polymers, could be calculated from ultrasonic measurements.

\section{ZINC OXIDE AND ALUMINUM NITRIDE THIN FILMS}

\section{Chairperson: S. LIEBERMAN}

P-1 High Deposition Rate Sputtered Zno Thin Films for BAW and SAW Applications, ${ }^{\mathrm{I}} \mathrm{Ph}$. DEFRANOULD, Thomson-CSF DASM Cagnes/Mer-06802-France. 
$C$-axis oriented $\mathrm{ZnO}$ thin films were grown with a DC magnetron sputtering equipment called "S-GUN" built by VARIAN. Reactive sputtering was performed in pure oxygen with a zinc target. Film quality has been studied on various substrates (sapphire, glass, fused silica, oxidized silicon) as a function of the deposition parameters: deposition rate, substrate temperature from $200^{\circ}$ to $500^{\circ} \mathrm{C}$, gaz pressure from 2 to 30 millitorrs. High deposition rate $(8-10 \mu / \mathrm{h})$ has been obtained with a minimum oxygen pressure of 2.5 millitorrs, a DC power of 1.5. $\mathrm{kW}$ and a target-substrate distance of $70 \mathrm{~mm}$. The film structure has been evaluated by $X$-ray diffraction and rocking curve and by scanning electron microscope. Standard deviation $\sigma$ of $C$-axis orientation around substrate normal better than $1^{\circ}$ have been measured. The electroacoustic properties of the film are evaluated for both bulk and surface waves. So coupling coefficient up to 90 percent of theoretical values have been measured on different structures. SAW propagation loss around $1 \mathrm{~dB} / \mu \mathrm{s}$ at $115 \mathrm{MHz}$ has also been estimated through delay line and optical probe measurements.

${ }^{1}$ This work was sponsored by DRET France.

P-2 ZnO System Preconditioning for Microwave Bulk Acoustic Delay Lines, H. L. SALVO and R. N. SUNDELIN, Westinghouse Defense and Electronics System Center, Baltimore, MD 21203, B. R. MCAVOY, Westinghouse Research and Development Center, Pittsburgh, PA 15235, and S. LIEBERMAN, Harry Diamond Laboratory, Washington, DC.

It is possible that there exists a system "memory" effect in ZnO sputtering systems which tends to mask the true results of a systematic determination of optimum sputtering parameters. As part of a program to increase $\mathrm{ZnO}$ quality for use in microwave bulk acoustic delay lines, we attempted to use such a series of experiments to define the optimum sputtering parameters for our RF diode system. We have found that the quality of the $\mathrm{ZnO}$ film seems to depend on the system conditions used in the previous runs. Although we do not understand which component of the system contains the memory element, a preconditioning of the system by presputtering in pure oxygen seems to reduce its effect. Using this preconditioning we have been able to produce $6 \mathrm{~dB}$ conversion loss transducers operating at approximately $4 \mathrm{GHz}$.

P-3 Post-Deposition Annealing of Zinc Oxide Films, F. S. HICKERNELL, Motorola Inc., Government Electronics Division, Scottsdale, AZ 85252 .

Post-deposition thermal and laser annealing of zinc oxide films has been investigated for the purpose of improving their acoustical and optical properties. Thermal annealing was found to reduce tensile stress and increase crystallographic ordering but there was little improvement in the film's acoustical and optical properties. Laser annealing showed only minor changes in strain and crystallographic ordering but two orders of magnitude improvement in the optical waveguide propagation loss property. ${ }^{1}$ Experimental evidence indicates that laser annealing is a more selective thermal process which reduces defects in the $\mathrm{ZnO}$ film near the substrate boundary. This paper discusses the annealing experimentation with sputtered $\mathrm{ZnO}$ films and related changes in their acoustical, optical, and structural properties.

'S. Dutta et al., Appl. Phys. Lett., Aug. 1981.

P.4 Plasma-Enhanced Metalorganic Chemical Vapor Deposition of c-Axis Oriented and Epitaxial Films of $\mathrm{ZnO}$ at Low Substrate Temperatures, T. SHIOSAKI, T. YAMAMOTO, M. YAGI, M. SHIMIZU, and A. KAWABATA, Department of Electronics, Faculty of Engineering, Kyoto University, Kyoto, Japan.

Zinc oxide films with the highly-oriented $c$-axis normal to the substrate surface are grown on glass substrates by plasmaenhanced metalorganic chemical vapor deposition of diethylzinc at substrate tempera- tures of $200^{\circ}-300^{\circ} \mathrm{C}$ at RF input powers higher than $220 \mathrm{~W}\left(0.7 \mathrm{~W} / \mathrm{cm}^{2}\right)$ in carbon dioxide or oxygen atmosphere. Carbon dioxide is the more preferable to oxygen because of its less inactivity on diethylzinc (or zinc) in the absence of the assist of the plasma. An epitaxial (1120) $\mathrm{ZnO}$ films are also grown on (0112) sapphire substrates at RF input powers higher than $50 \mathrm{~W}$ at substrate temperatures higher than $200^{\circ} \mathrm{C}$. $\mathrm{X}$-ray analy ses have shown that carbon dioxide and diethylzinc (or zinc) do not react without the assistance of the plasma at $20^{\circ} \mathrm{C}$ to $300^{\circ} \mathrm{C}$. When applied input RF power is lower than $200 \mathrm{~W}$, the $\mathrm{ZnO}$ (101) peak is also observed although the (002) peak intensity increases with increasing the RF input power. At RF input powers higher than $220 \mathrm{~W}$ the (101) peak is not observed. The $c$-axis orientation degree is also increased with increasing the substrate temperature, especially around $200^{\circ} \mathrm{C}$ which is zinc decomposition temperature from diethylzinc. The deposition rate is $2 \mu \mathrm{m} / \mathrm{h}$ at the substrate temperature of $200^{\circ} \mathrm{C}$ at the input power of $220 \mathrm{~W}$. The resistivity of the as-grown plasma-enhanced CVD film is about $10^{3} \Omega \mathrm{cm}$.

P-5 Sputtered AIN Films for Bulk-Acoustic-Wave Devices, ${ }^{1} \mathrm{~J}$. S. WANG and K. M. LAKIN, Ames Laboratory-USDOE, Iowa State University, Ames, IO 50011.

AlN films are of significant interest for high frequency bulk-acousticwave (BAW) devices because of their high acoustic wave velocity and fairly high electromechanical coupling coefficients. In this study, $\mathrm{Si}$ is used as the substrate because of its future in integrated circuit devices. The AlN films are prepared at low temperature by using reactive DC magnetron sputtering. Highly oriented films on Si are obtained with a standard deviation angle $\sigma=0.9$ in the x-ray rocking curve. A pure aluminum (99.999) target is used and sputtering is done in a lowoxygen $(<0.5 \mathrm{ppm})$ nitrogen atmosphere. Typical sputtering conditions are: $\mathrm{DC}$ power $=100 \mathrm{~W}$, sputtering rate $=0.6 \mu \mathrm{m} / \mathrm{h}$, substrate temperature $=100-400^{\circ} \mathrm{C}$ and nitrogen pressure $=3 \mu \mathrm{m}$. These films are evaluated by SEM, x-ray diffraction and BAW measurements. Surface crazing of the AIN films on Si were observed. Composite resonators of AIN/Si and bulk wave transducers were fabricated for BAW measurement and film evaluation. The acoustic resonant responses were detected at fundamental frequency of $387 \mathrm{MHz}$, second overtone frequency of $799 \mathrm{MHz}$, and third overtone frequency of $1271 \mathrm{MHz}$.

${ }^{1}$ This work supported by the Air Force Office of Scientific Research.

P-6 Low-Temperature Growth of the Piezoelectric AlN Film and its Optical and Acoustical Properties, T. SHIOSAKI, K. HARADA, and A. KAWABATA, Department of Electronics, Faculty of Engineering, Kyoto University, Kyoto, Japan.

By using RF planar magnetron sputtering, piezoelectric AlN films with the $c$-axis highly oriented normal to the substrate surface can be grown on sapphire, silicon and glass substrates at the substrate temperature as low as $20^{\circ} \mathrm{C}$. The target is 99.99 percent aluminum plate and the atmospheric gas is $\mathrm{Ar}+\mathrm{N}_{2}(1: 1)$. In addition to the preliminary results reported in the last symposium, the following optical and acoustical properties have been obtained. The measured refractive index $n_{0}$ is 2.060 . The optical guided wave loss is $10.6 \mathrm{~dB} / \mathrm{cm}$ for $\mathrm{TE}_{0}$ mode in a $0.817 \mu \mathrm{m}$ thick film on a glass substrate. Infrared optical reflection spectrum measurement of a $110 \mu \mathrm{m}$ thick AlN film

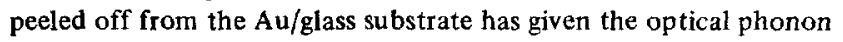
properties of $\lambda_{L 0}=11.0 \mu \mathrm{m}, \lambda_{T 0}=15.3 \mu \mathrm{m}, \epsilon_{\infty}=4.31, \epsilon_{0}=8.34$, and the longitudinal phonon velocity along $c$-axis of $1.38 \times 10^{4} \mathrm{~m} / \mathrm{s}$. The effective SAW coupling factor $k^{2}$ on silicon with the IDT located on the top of AIN film is $0.15-0.21$ percent at the wavenumber times film thickness $(\mathrm{KH})$ of $0.5-2.0$. The temperature coefficient of delay of SAW propagation on (001) $\mathrm{Si}$ is $23.9 \mathrm{ppm} /{ }^{\circ} \mathrm{C}$ at $\mathrm{KH}=1.84$. The AlN film sputtered on a Mylar (polyester) film with its one surface metalized has given a response as a loud speaker at audio frequency. 


\section{ACOUSTOOPTIC DEVICES AND SIGNAL PROCESSING I}

Chairperson: D. L. HECHT

Q-1 Acoustooptic Spectrum Analysis for Electronic Warfare Applications, M. C. HAMILTON, Avionics Laboratory, AFWAL/AADO, Wright-Patterson AFB, OH 45433.

In response to increasingly dense signal environments, electronic warfare engineers are evaluating next generation receiver technologies that can process multiple, simultaneous signals over large instantaneous bandwidths. The inherent parallel processing nature of acoustooptic spectrum analysis makes this approach to signal processing an attractive candidate for inclusion in future EW systems. The present performance levels of lasers, Bragg cells, and detectors are close to that necessary for meeting the minimum system requirements. The small space on aircraft for EW systems has motivated the placement of emphasis on miniaturization of AO spectrum analyzers. Two parallel paths are being followed-the exploration of concepts for a "mini-bench" of discrete components and the development of integrated optics technology. While the integrated optics approach certainly has the edge in elegance, it remains unclear as to which is the most cost- and size-effective solution. Those characteristics of each that need improvement are discussed and the key tradeoffs between the two are examined.

Q-2 Performance of the Integrated Optic Spectrum Analyzer, ${ }^{1}$ T. R. JOSEPH, Hughes Aircraft Company, Fullerton, CA 92634, and T. R. RANGANATH and J. Y. LEE, Hughes Research Laboratory, Malibu, CA 90265.

The integrated optic spectrum analyzer is a device which utilizes the Bragg interaction between a SAW and a guided optical beam to perform spectral analysis. Hughes has developed the first fully integrated IOSA which uses a butt-coupled GaAlAs semiconductor laser, a $\mathrm{LiNbO}_{3}$ waveguide substrate, a $400 \mathrm{MHz}$ bandwidth SAW transducer, and a silicon detector/CCD array chip. The completed device exhibited a 3 $\mathrm{dB}$ bandwidth of $380 \mathrm{MHz}$ with 10 percent/watt deflection efficiency at $\lambda_{\mathrm{opt}}=0.82 \mu \mathrm{m}$. Detailed measurements of performance including dynamic range, $R F$ resolution, and pulsed response will be presented. The SAW transducer was a modified chip transducer with electrodes that are continuously tilted to obtain broadband matching to the Bragg condition. Investigations are continuing to achieve larger operating bandwidths using this transducer type. Two $500 \mathrm{MHz}$ band width transducers with $1 \mathrm{GHz}$ center frequencies have been developed. A fundamental mode transducer with a midband insertion loss of $14 \mathrm{~dB} /$ transducer has been tested. Initial deflection measurements indicate an acoustooptic interaction bandwidth of more than $400 \mathrm{MHz}$. The second transducer uses a third harmonic double electrode design. It exhibits midband insertion loss of $20 \mathrm{~dB} /$ transducer. Acoustooptic measurements are currently underway on that design. The results of those efforts will be presented.

${ }^{1}$ This work supported in part by the Air Force Avionics Laboratory, under Contract No. F33615-78-C-1450.

†Q-3 An Advanced Integrated Optical RF Spectrum Analyzer, D. MERGERIAN, E. C. MALARKEY, R. P. PAUTIENUS, J. C. BRADLEY, A. L. KELLNER, and M. D. MILL, Westinghouse Systems Development Division, Baltimore, MD 21203.

The performance of an improved version of the integrated optical $R F$ spectrum analyzer will be described. The spectrum analyzer is a threeelement hybrid assembly in which a CW GaAlAs laser and a 140-element high-speed photodiode array are butt-coupled at opposite ends of a $\mathrm{LiNbO}_{3}$ integrated optical substrate containing the SAW-guided optical wave Bragg cell and aspherically-corrected, diffraction-limited geodesic input and output lenses. The device divides a $400 \mathrm{MHz}$ instantaneous $\mathrm{RF}$ bandwidth centered at $600 \mathrm{MHz}$ into $4 \mathrm{MHz}$ channels and reports a completely updated RF spectrum every two micro-seconds. It is capable of detecting multiple simultaneous pulses of durations as short as $0.1 \mu \mathrm{s}$. The improved optical system is designed to yield a linear, spurious-signal-free dynamic sange of $50 \mathrm{~dB}$ and to detect simultaneous signals differing in strength by $u p$ to $40 \mathrm{~dB}$ at a frequency separation of only $16 \mathrm{MHz}$.

Q-4 The IO Spectrum Analyzer: An Emerging Technology, R. L. DAVIS and F. S. HICKERNELL, Motorola Inc., Government Electronics Division, Scottsdale, AZ 85252 .

Recent advances made in RF spectrum analysis using integrated optic and surface acoustic wave technologies will impact future signal processing systems. Described in this paper is a contribution to this emerging technology through the fabrication and performance evaluation of a planar guided wave spectrum analyzer developed on lithium niobate with hybrid coupled laser source and photodetector array. The unique features of this development are 1) the use of high index low-loss thin film $\mathrm{Nb}_{2} \mathrm{O}_{5}$ lenses for collimation and Fourier transformation, 2) the use of a commercially available single mode laser diode source and a silicon photodetector array with serial read-out and 3) a broadband transducer approach with demonstrated acoustooptic diffraction capabilities into the $1.5 \mathrm{GHz}$ region. The IO-SAW device has been engineered for fabricational simplicity, using standard IC processing techniques amenable to low-cost volume production. As performance improvements are made in the technologies associated with the IOSA, they can be easily implemented in the design concept described.

Q-5 Bragg Cell Signal Processing Advances, M. W. CASSEDAY, N. J. BERG, and I. J. ABRAMOVITZ, Harry Diamond Laboratories, Adelphi, MD 20783.

Several recent developments may extend the performance of Bragg cell spectrum analyzers and may allow more complete exploitation of the Fourier transform properties of these acoustooptic devices. These developments include the discovery of an ultrabroadband surface-wave acoustooptic interaction in $Y-Z$ lithium niobate; the construction of an optically matched fiberoptic array coupled to discrete photo-diodes for fast, large dynamic range, parallel readout detection at the Fourier plane; and the use of optical heterodyne techniques in conjunction with the fiber array for reconstruction and adaptive filtering of the input signals. The broadband interaction with surface waves will allow greater interaction efficiency for a given bandwidth, with the low transform phase distortion associated with the surface acoustic wave acoustooptic interaction in $Y-Z$ lithium niobate. The fiberoptic array allows fraction of a microsecond resolution of frequency agile signals over a $50 \mathrm{~dB}$ or greater dynamic range; and by combining coherent heterodyne detection with this array, it is possible to achieve signal filtering such as narrow-band interference excision from broadband signals.

\section{PHOTOACOUSTICS: SPECTROSCOPY AND NDE}

\section{Chairperson: A. ROSENCWAIG}

R-1 Reactions in Polymers Studied with Fourier Transform Infrared Photoacoustic Spectroscopy, ${ }^{1}$ B.S.H. ROYCE and Y.C. TENG, Applied Physics and Materials Laboratory, Princeton University, Princeton, NJ 08544 .

Foutier transform infrared photoacoustic spectroscopy has been used to obtain optical absorption spectra of polymeric systems during cure. Data will be presented on the photopolymerization of acrylic materials and a comparison to transmission FTIR data on the same materials will be made. Highly light scattering materials are not 
amenable to transmission measurements and under the circumstances the use of photoacoustic spectroscopy is advantageous This capability will be illustrated with PAS spectral data on RTV silicon rubbers loaded with $\mathrm{SiO}_{2}$ and $\mathrm{TiO}_{2}$.

${ }^{1}$ This work was supported in part by ARO contract DAAG 28-80-C0053.

R-2 Photoacoustic and Photoluminescence Measurements of Defects in Semiconductors, N. MIKOSHIBA and K. TSUBOUCHI, Research Institu te of Electrical Communication, Tohoku Univ., Katahira 2-1-1, Sendai 980 , Japan.

Photoacoustic (PA) and photoluminescence (PL) measurements were carried out simultaneously at room temperature in order to get a new spectroscopic information on defects in semiconductors. PA signal was detected with a piezoelectric transducer $(\mathrm{ZnO})$ coated by sputtering onto the back surface of the sample. At first, simultaneous measurements of PA and PL on GaAs, n-InP, and p-InP showed that the PA signal levels of GaAs, $p$-InP, and $n$-InP were in order of magnitude, while the order of the PL signal levels was just opposite. Next, we measured the change in time of the PA and PL signals when the $\mathrm{Kr}^{+}$ laser of $\sim 3 \mathrm{~kW} / \mathrm{cm}^{2}$ irradiated the surface of the sample. We observed an increase of PA signal with a decrease of PL signal in GaAs, but in InP we could not observe any correlation between the changes of $P A$ and PL signals. In GaAs, after $3 \mathrm{~h}$ of $\mathrm{Kr}^{+}$laser irradiation, we observed that a PA signal peak due to dislocation and another peak due to the oxidation in the crystal surface grew up. The oxidation effects on PA and PL were also confirmed by the following experiments. When the GaAs sample was held in an $\mathrm{O}_{2}$ atmospiere, the oxidation peak of PA signal grew up, while the PL peak decreased. By etching the crystal surface within about $3000 \AA$, the PA and PL signal returned back to the initial level before oxidation. Finally, we present the simple theoretical model of PA mechanism for the local heating in the dislocation and the oxidized layer.

R-3 A General Theorem for Simplifying Calculations of Photoacoustic Signals in Gas Filled Cells,' P. K. KUO, L. J, INGLEHART, L. D. FAVRO, and R. L. THOMAS, Wayne State University, Detroit, MI 48202 .

We prove a general theorem which states that the photoacoustic signal generated by a localized source in a gas filled cell is equivalent to the surface temperature distribution which would be produced if the sample were uniformly illuminated. The theorem applies to an extended source as well, and permits completely general sample/cell geometry, including surface shape and internal structure. We compare calculations based on this theorem with experimental data for simple geometries.

${ }^{1}$ This research was sponsored by ARO under Contract No. DAAG29. 79-C-0151

R-4 Applications of Laser-Generated Ultrasound in Metals, D. A. HUTCHINS, ${ }^{1}$ R. J. DEWHURST, and S. B. PALMER, Department of Applied Physics, University of Hull, Hull HU6 7RX, U.K.

A detailed investigation has been carried out by the above authors to understand the mechanisms of generation of ultrasound in metals irradiated with $Q$-switched laser pulses. As a result of this work, various applications of the technique have been highlighted. The primary use is as a remote method of generation, for particular application to nondestructive evaluation in hostile environments; the flexibility of the source in this role will be outlined, with particular attention being paid to the directivity of the longitudinal and shear modes and their efficiency of generation. Other potential uses are as a standard acoustic source with excellent repeatibility, and as a convenient method for liquid depth estimation.

${ }^{1}$ Present address: Technical University of Nova Scotia, P.O. Box 1000 , Halifax, N.S. B3J $2 \times 4$, Canada.

$\dagger$ R-5 Acoustic Techniques For Characterization of Ablative Processes in Composite Structures, C. E. YEACK and R. L. MELCHER, IBM T. J. Watson Research Center, Yorktown Heights, NY 10598, and H. E. KLAUSER, IBM System Products Division, Endicott, NY 13760.

A real time nondestructive acoustic monitoring scheme has been developed to analyze and control localized thermal ablation of composite structures. Analysis of photoacoustic signals obtained from successive equal energy pulses incident on the same region of a complex structure gives information regarding local inhomogeneities and defects. The integrated acoustic signal is proportional to the amount of optical energy absorbed from the incident pulse, which means that regions not absorbing the incident energy, such as voids, defects, and optically reflecting objects, do not contribute to the acoustic signal. As in conventional photoacoustics, the acoustic signals measured are the result of optical absorption which gives rise to a temperature profile at the surface of the absorbing medium. During heat transfer to the surrounding gas, the gas expands and generates an acoustic wave which propagates to and is detected by an appropriate acoustic sensor. However, unlike conventional photoacoustics, experiments reported here have all been done using pulsed, rather than $\mathrm{CW}$, excitation and have not required the use of an airtight acoustic cell. A statistical analysis of the photoacoustic signals from an extensive set of experiments on a composite material will be presented.

R-6 Photoacoustic Blast Wave in a Metal Vapor: Generation and Detection, A. C. TAM, W. ZAPKA, K. CHIANG, and W. IMAINO, IBM Research Laboratory, Dept. K46/282, San Jose, CA 95193.

Photoacoustic pulse generation via breakdown is achieved in dense Cesium metal vapors or vapor pressures ranging from 2 to 100 torr, by using a dye laser pulse of energy variable from $10^{-6}$ to $10^{-3} \mathrm{~J}$, and tuned to the $\mathrm{C}_{\mathrm{S}}$ transition at $6010 \mathrm{~A}$. The acoustic pulse propagation is detected by the transient photorefractive deflection of a $\mathrm{CW}$ probe laser beam that is displaced from but parallel to the pulsed laser beam. The temperature-dependent velocity of infinitesimal ultrasonic waves in a corrosive metal vapor is measured for the first time. The supersonic propagation of finite amplitude acoustic pulses (blast waves) obtained with a higher laser pulse energy is also studied; our data, with Mach numbers ranging from 2.1 down to below 1.01 , agree surprisingly well with the prediction of Vlases and Jones for cylindrical blast waves; this provides a new experimental support for their theoretical trajectory formula for blast waves in the extremely weak amplitude limit.

R-7 Mechanical Excitation of a Membrane by an Optical Beam, E. DIEULESAINT, D. ROYER, and C. BONNEFOY, Laboratoire d'Acoustoélectricité de l'Université Pierre et Marie Curie, 10, rue Vauquelin, 75231 Paris Cedex 05.

Mechanical or electromechanical resonators, such as metallic bars or piezoelectric plates, play a major role in telecommunication and instrumentation domains, particularly in the filtering devices. Their success arise from the fact that they are easily excited with an electrical signal (directly or with a transducer). Will their role remain essential now that optical techniques enter into these domains? It seems to us that the answer to this question depends partly on the optical methods which will be found to make these resonators vibrate. One solution could be the photoacoustic effect. We have carried out first experiments with membranes excited by a modulated optical beam. Silicon membranes of thickness about $10 \mu \mathrm{m}$, of diameter a few millimeters, 
were excited by an acoustooptically modulated laser or a directly modulated intensity diode. Results will be given in the form of impulse or continuous responses $(f=10 \mathrm{KHz})$. These responses were detected by the deviation of a narrow light beam reflected by the vibrating membrane.

\section{BULK RESONATORS AND FERROELECTRICS}

\author{
Chairperson: G. W. FARNELL
}

S-1 Laser Probe Investigation of Gujded Acoustic Interface Waves In Differentially Poled Ceramics, B. A. AULD and H. A. KUNKLE, Edward L. Ginzton Laboratory, Stanford University, Stanford, CA 94305 .

Guided acoustic waves on "interfaces" between regions of opposite net electric polarization in piezoelectric ceramics have been predicted by Maerfeld, Tournois, and Bleustein. Such waveguide structures have been realized by counterpoling selected regions of an originally uniformly poled ceramic, with the interface occurring at the boundary between poled and counterpoled regions. We have used the Ash laser heterodyne probe technique to investigate the nature of these guided waves in ceramic plates. The structures investigated include an artificial "glue-bond" interface between two oppositely-poled ceramic bars, and interfaces occurring in actual differentially poled ceramics. The laser probe is used to study the properties of interface modes (wavelengths and transverse decay rates) by scanning over the ceramic structure, thereby measuring the particle displacement field.

S-2 Ferroelectric/Paralectric SAW Transduction in Strontium Barium Niobate, E. J. STAPLES and R. R. NEURGAONKAR, Rockwell International, Thousand Oaks, CA 91360.

The tetragonal $\mathrm{Sr}_{0.61} \mathrm{Ba}_{0.39} \mathrm{Nb}_{2} \mathrm{O}_{6}$ solid solution belongs to the tungsten bronze structural family and has a Curie temperature of $70^{\circ} \mathrm{C}$. Below the Curie temperature spontaneous polarization along the $c$-axis gives rise to piezoelectric coupling terms $d_{31}, d_{33}$, and $d_{15}$. Above the Curie temperature the solid solution enters the paraelectric phase where application of an external electric field is used to induce polarization and generate surface acoustic waves. For example $Y$-cut, $z$-propagating surface waves can be launched by simultaneously applying a poling field along with the applied RF potential to the interdigital electrodes. Surface waves in this case are electrostrictively generated. Electrostrictive surface wave generation on a paraelectric crystal offers some advantages and disadvantages over normal operation in the ferroelectric regime. Advantages include twice the frequency for the same electrode periodicity and the ability to program the transducer amplitude and phase simply by applying the appropriate voltage polarity. The primary disadvantage is the need for oven control to maintain the paraelectric temperature. This might be overcome by using materials with Curie temperatures below room temperature.

S-3 [Invited] The SC-cut Crystal-An Overview, J. A. KUSTERS, Hewlett Packard Co., Santa Clara, CA 95050.

The SC cut was first described theoretically by Holland at the 1974 Ultrasonics Symposium. ${ }^{1}$ Since then, extensive experimental and theoretical work has verified the existance of this cut and demonstrated its superiority in thermal transient response and in planar stress compensation. This paper will review the work done to date with emphasis on the improvements in precision frequency standards offered by the $\mathrm{SC}$ cut, and comparisons between the $\mathrm{SC}$ and other standard crystal cuts. Difficulties encountered in making and using SC cuts will also be discussed. The paper will conclude with a discussion of digital fre- quency compensation based upon dual-mode operation of SC bulkwave resonators.

${ }^{1}$ R. Holland, "Nonuniformly heated anisotropic plates: Il, Frequency transients in AT and BT quartz plates," 1974 Ultrason. Symp. Proc., pp. 592-598.

S-4 Resonators with Reduced Frequency Sensitivity to Static and Dynamic Stresses, M. NAKAZAWA, Princeton University, Princeton, NJ 08540, T. LUKASZEK and A. BALLATO, Electronics Technology \& Devices Laboratory, Fort Monmouth, NJ 07703.

We have investigated plate resonator configurations that are amenable to VHF-UHF operation with equivalent circuit parameters not achiev. able with SAW resonators, and that give good promise both of providing very stable frequencies irrespective of static boundary and dynamic body stresses, as well as being capable of miniaturization and integration with microelectronic circuitry. These BAW resonators consist of "energy-trapped" resonant membranes embedded in monolithic support structures that bear most of the boundary stresses (often thermo elastic). Dynamic body stresses, arising from the accelerations of shocks and vibrations, are reduced in influence by the double-contilever stiffening of the vibrating element by the support. We discuss the relative merits of the different configurations, and give the results of both static and dynamic stress experiments, wherein improvements of a factor of ten and more have been observed. Also reported are nonlinear frequency-acceleration effects found at high $g$ levels.

S-5 Transient Testing of Transducer Bonds, ${ }^{1}$ A. BANAH, A. KOR$P E L$, and R. VOGEL, Department of Electrical and Computer Engineering, The University of lowa, lowa City, IA 52242.

Conventionally the quality of transducer bonding is tested by measuring the frequency response. We present theoretical and experimental aspects of a more direct technique, which uses impulse excitation of an untuned bonded transducer. In conjunction with the technique we use a novel model of the transducer as a reentrant transmission line.

${ }^{1}$ This work was supported in part by the National Science Foundation under Grant ENG-7902889.

\section{ACOUSTOOPTIC DEVICES AND SIGNAL PROCESSING II}

\section{Chairperson: T. R. JOSEPH}

†T-1 Acoustic Properties in Bulkwave Acoustooptic Spectrum Analysis, D. L. HECHT, Xerox Corporation, 3333 Coyote Hill Rd., Palo Alto, CA 94304.

The acoustic properties of the acoustooptic medium are critical factors in acoustooptic spectrum analysis (AOSA) performance. Velocity determines frequency resolution for a given physical aperture. The shape of a region of the material slowness surface determines the frequency plane blur function and frequency scale distortion. ${ }^{1}$ Minimum dispersion effects occur at extrema of the slowness surface with minimum curvature. ${ }^{2}$ High velocity minimizes frequency distortion. Acoustic attenuation limits aperture bandwidth combinations. High photoelastic constants are key factors in practical devices.

'I. C. Chang and D. L. Hecht, SPIE, vol. 241, July 1980, pp. 129-138.

${ }^{2}$ D. L. Hecht and G. W. Petrie, 1980 IEEE Ultrasonics Symp. Proc., pp. 474-478.

T-2 Wideband Acoustooptic Bragg Cells, I. C. CHANG, G. PETRIE, and R. CADIEUX, Applied Technology Division of Itek Corporation, Sunnyvale, CA 94086. 
Wideband acoustooptic Bragg cells play a key role in the real time optical signal processors, e.g., the acoustooptic spectrum analyzers. The critical issue has been the relatively low diffraction efficiency obtainable with state-of-the-art $\mathrm{GHz}$ bandwidth Bragg cells ( $<1$ percent/ watt). A higher efficiency is desired for greater dynamic range and increased sensitivity. This paper presents recent progress of efficient wideband Bragg cells development. Optimization of device performance has been achieved by the selection of acoustooptic materials and modes, the improved transducer fabrication technology, and the use of performance enhancement techniques. The two enhancement techniques studied are birefringent diffraction and acoustic beam confinement. Experimental results were obtained with a $\mathrm{LiNbO}_{3}$ device using an $x$-polarized shear wave operated in the tangential phase matching birefringent diffraction mode. The device demonstrated a 3-dB bandwidth of $1.2 \mathrm{GHz}(1.8-3 \mathrm{GHz})$ and a diffraction efficiency of three percent per RF watt at $0.633 \mu \mathrm{m}$. Experimental results obtained with several other $\mathrm{GHz}$ bandwidth Bragg cells utilizing different materials will also be presented.

T-3 Wideband Interferometric Acoustooptic Bragg Cell Spectrum Analyzer, M. L. SHAH, J. R. TEAGUE, R. V, BELFATTO, and D. W. THOMSON, and E. H. YOUNG, Harris Corporation, Melbourne, FL 32901.

An interferometric acoustooptic spectrum analyzer system is configured in which light signal is made proportional to the amplitude of the signal, thereby doubling the dynamic range in $\mathrm{dB}$ over the Bragg cell spectrum analyzer. In an experimental system 40 percent increase in DR was observed. Several schemes to generate reference beam and obtain a constant frequency interference beats are discussed. Theoretical and experimental DR of noninterferometric and interferometric acoustooptic Bragg cell spectrum analyzer is computed. Experimentally, we have observed $58 \mathrm{~dB}$ dynamic range for a cw signal and 54 $\mathrm{dB}$ dynamic range for five $\mu$ s pulse signals with a 100 percent probability of intercept and less than $1 \mu$ s time-of-arrival accuracy. The system bandwidth was close to $250 \mathrm{MHz}$ with $3.5 \mathrm{MHz}$ frequency resolution. The spurious level was below $50 \mathrm{~dB}$ and we used a five $m W$ HeNe laser in Mach-Zehnder configuration. Discrete silicon photodetectors with a fiber optic array were used in the experiment.

†T-4 Interferometric Bragg Cell Spectrum Analyzer, M. L. SHAH, E. H. YOUNG, and A. VANDER LUGT, Harris Corporation, Melbourne, FL 32901 ; M. HAMILTON, AFAL, Dayton, OH 45433.

An interferometric spectrum analyzer system requires a reference waveform at every frequency position of a signal. The reference can be present all the time or may have some duty factor. Generation of the waveform will be discussed as well as interferometric schemes based upon beam splitting and combining. Reference signal generation, which could be a linear chirp or a pseudonoise sequence, will be discussed to determine its implementation feasibility for the system. The preferred scheme for the experiment will be discussed in detail and the proposed configuration will be presented. The light incident on the acoustic cell was analyzed relative to Gaussian and sinc function spot profiles. The required number of detectors and fiber optics array will be discussed. The chosen design was analyzed for determining if a sufficient dip existed between two tones separated by one frequency resolution element. A computer program was developed to simulate and analyze the output optical spectrum. Self-interference resulting from interference among reference spots will be analyzed and discussed as well as methods to minimize their effects. In addition, spill-over image frequency terms will be discussed. The predicted performance of an interferometric acoustooptic spectrum analyzer system, which could be as large as $60 \mathrm{~dB}$ for our proposed system, will be presented.

T-5 Surface-Wave Acoustooptic Cepstrum Processor for Characterization of Wideband Signals, I. J. ABRAMOVITZ, N. J. BERG, M. W.
CASSEDAY, and J. J. CECCONI, Harry Diamond Laboratories, Adelphi, MD 20783.

A modified Cepstrum processor has been implemented that uses a surface acoustic wave (SAW) delay line, an integrating photodiode array, and a digital inverse Fourier transformer. This hybrid analog/ digital system can characterize wideband signals in the presence of noise and/or high-power narrowband signals. A 30-MHz bandwidth SAW delay line operating at $90-\mathrm{MHz}$ and a telescope system produce an acoustooptic Fourier transform of an input signal consisting of the sum of two similar signals with a time delay between them. An integrating photodiode array at the Fourier transform plane detects the optical transform which is then analog-to-digital converted. Saturation of only some photodiode array elements occurs for high-power narrowband signals. A digital inverse Fourier transform is performed on this output. Information including relative signal time delay, band width, frequency, and modulation type is obtained from the Fourier transform and its inverse. Signal-to-noise enhancement and frequency/time resolution of the modified Cepstrum processor are comparable to the acoustooptic time-integrating correlator previously reported; however, the advantage of the new system is its relative immunity to saturation from high-level cw-like signals. An improvement of almost $20 \mathrm{~dB}$ in high-level saturation immunity has been observed vis-á-vis the timeintegrating correlator. This processor has the potential for handling much larger bandwidths.

T6 Second Order Bragg Diffraction Operation of Acoustooptic Devices, ${ }^{1}$ T. C. POON and A. KORPEL, Department of Electrical and Computer Engineering, University of Iowa, lowa City, Iowa, IA 52242.

Conventional Bragg diffraction devices operate in the first order with the light incident at the Bragg angle. Certain advantages would accrue, such as larger deflection angles and frequency shifts, if reasonably efficient operation could be obtained in the second order. In this talk we present both theoretical and experimental details of such a mode of operation with 70 percent efficiency and $>20$ percent bandwidth.

${ }^{1}$ This work was supported by the National Science Foundation under Grant ENG-7902889.

\section{ACOUSTIC MICROSCOPY: MATERIAL CHARACTERIZATION}

\section{Chairperson: L. W. KESSLER}

U-1 Acoustic Microscopy of Slowness Surfaces, ${ }^{1}$ I. R. SMITH, D. A. SINCLAIR, and H. K. WICKRAMASINGHE, Department of Electronic Engineering, University College, London, UK.

Acoustic micrographs reveal variations in the mechanical properties of a specimen with high spatial resolution. However, the image contrast is derived from a complex interplay of variations in the specimens elasticity and density. We have developed a method by which the variations of a single parameter may be isolated to form an image. In this, the far field diffraction pattern of a thin plate specimen, illuminated by a focused acoustic source, is directly related to the slowness surfaces of the specimen in the narrow focal region, generally only a few wavelengths across. The slowness surfaces are recorded for each image point and may be used to calculate the density, longitudinal, and shear elasticity and thickness of the specimen at each point. The structure and orientation of anisotropic specimens may also be revealed in this way.

${ }^{1}$ This work was supported by the Medical Research Council.

U-2 An Acoustic Microscope for Surface Coating Characterization, ${ }^{1}$ B. W. MAXFIELD, L-333 Lawrence Livermore National Laboratory, 
Livermore, CA 94550 and R. O. WEGLEIN, Hughes Aircraft Company, Culver City, CA 90230.

Acoustic microscopy has been used recently for nondestructive thickness measurements of surface layers. The approach is based upon the thickness dependence of the surface wave velocity in a layered medium. Using an acoustic material signature from a focused-beam microscope as described in the literature one can determine the surface-wave velocity. This technique also has the potential of providing information on coating defects and surface adhesion. An experimental facility is being established at LLNL for the characterization of the relatively thick coatings that are frequently used within the engineering community for joining materials and protecting surfaces from corrosion and erosion. Thicknesses in the range of 0.01 to $0.25 \mathrm{~mm}$ require an operating frequency between 20 and $200 \mathrm{MHz}$. A coherent detection system capable of operation at discrete frequencies between these limits has been constructed and initially used to measure the characteristics of silver on stainless steel and copper on fused quartz.

${ }^{1}$ This work was performed under the auspices of the U.S. Department of Energy by Lawrence Livermore Laboratory under Contract W-7405-Eng-48.

U-3 Integrated Circuit Inspection via Acoustic Microscopy, R. D. WEGLEIN, Hughes Missile Systems Group, Canoga Park, CA 91304.

The reflection acoustic microscope provides a unique capability of nondestructive inspection and characterization of subsurface defects. The vehicle for imaging subsurface structure is a two-level hybrid integrated circuit of "flip-chip" construction. The two semiconducting chips, one Si and one InSb, that form the hybrid, are optically opaque, thus excluding conventional forms of microscopy from post-assembly inspection. After a brief description of the experimental instrument, operating near $400 \mathrm{MHz}$, the formation of image contrast arising through both longitudinal and Rayleigh wave reflection in wide-angle acoustic lenses is discussed. Acoustic images taken at increasing depth inside the flip chip that contains an array of photovoltaic detectors are examined and analyzed. Though the resolution decreases at greater depth approximately in accordance with published results; it is quite adequate to image essential subsurface features.

U-4 An Examination of Sedimentary Minerals and Rocks with the Scanning Laser Acoustic Microscope, J. BROWN and G. PIRIE, Schlumberger-Doll Research, Ridgefield, CT 06877.

Petrophysical characterization of sedimentary rocks is of interest to the petroleum industry in unraveling the origin, migration, and entrapment of hydrocarbons. Optical characteristics of sedimentary minerals and rocks are routinely observed with the petrographic (polarizing) microscope and reported in the literature; an acoustic examination at comparable magnification (i.e., about 85 times) has been reported only once. ${ }^{1}$ We present quantitative measurements of the ultrasonic velocities of selected individual mineral grains and compare these with published values obtained using macroscopic techniques. Qualitative interpretation of the acoustic photomicrographs includes intergranular pore identification and distribution, mineral and rock grain size and shape, grain-to-grain contacts, and identification of sedimentary laminae and beds. The relative advantages and disadvantages of using the scanning laser acoustic microscope to characterize sedimentary minerals and rocks are discussed. For example, fractures and microfractures not observed optically can be identified acoustically. However, multiple twinning of plagioclase feldspar, which can be distinguished optically, are acoustically invisible.

'J. S. Danburg and D. E. Yuhas, Geophys. Res. Lett., vol. 5, p. 885, 1978.

†U-6 Acoustic Microscopy of Polymers, ${ }^{1}$ P. A. TUCKER, North Carolina State University, Raleigh, NC 27650.
Recent papers by Wilson and Tucker ${ }^{2}, 3$ have described the first applications of reflection acoustic microscopy (AC) to polymer materials. Typical samples investigated at $375 \mathrm{MHz}$ were polypropylene spherulites. Such micrographs resemble those obtained with polarized light, but they also provide additional information about the stiffness and planar structures of the specimens. Variations in morphology and structure change the relative phases of the collected waves, and the images have very high contrast. With $\mathrm{AC}$ it has been possible to obtain a detailed view of the interfibrillar growth of spherulites never before obtained. Bands analogous to Newton's Rings are obtained on drawn polymers. The "acoustic material signature" permits the controlling of a parameter analogous to light's "phase contrast" as demonstrated on crazed polystyrene. Other useful techniques appear feasible with AM, such as assessments of anisotropies and examination of thin polymer films which are transparent to light.

${ }^{1}$ This work partially supported by the North Carolina Board of Science and Technology.

${ }^{2}$ Paul Tucker and Robt. Wilson, J. Pol. Sci.: Poly. Lett. Ed., p. 18 , 1980 .

${ }^{3}$ Robt. Wilson and Paul Tucker, Appl. Phys. Lett., vol. 35, p. 15 , Nov. 1979.

\section{THIN FILM SAW DEVICES}

\section{Poster Session}

V-1 Sputtered Aluminum Nitride on Silicon for SAW Device Applications, ${ }^{1}$ L. G. PEARCE, R. L. GUNSHOR, and R. F. PIERRET, School of Electrical Engineering, Purdue University, West Lafayet te, IN 47907.

Reactive RF planar magnetron sputtering is employed to deposit piezoelectric aluminum nitride films on silicon substrates where the substrate temperature during deposition is below $300^{\circ} \mathrm{C}$. The films grown on substrates of both (100) and (111) oriented silicon, with and without the presence of a thermally grown oxide, are oriented with the $c$-axis normal to the substrate surface. We report the operating characteristics of several new AlN-on-silicon devices. These include two-port delay lines, degenerate monolithic convolvers, and surface acoustic wave resonators utilizing metal strip reflector arrays. The reported data includes electrical characteristics of the metal-Aln- $\left(\mathrm{SiO}_{2}\right)-\mathrm{Si}$ sandwich, and dispersion and electromechanical coupling properties for $(100)$-cut $/\langle 100\rangle$-propagating and $(111)$-cut $/\langle 2 \overline{1} \overline{1}\rangle$-propagating substrates.

${ }^{1}$ This work was sponsored jointly by the Air Force Office of Scientific Research Grant AFOSR-77-3304, the National Science Foundation Grant ECS-8103744, and the NSF-MRL Program.

V.2 AIN Material Constants Evaluation and SAW Properties on AIN/ $\mathrm{Al}_{2} \mathrm{O}_{3}$ and $\mathrm{AIN} / \mathrm{Si}, \mathrm{K}$. TSUBOUCHI, K. SUGAI, and N. MIKOSHIBA, Research Institute of Electrical Communication, Tohoku University, Katahira 2-1-1, Sendai 980, Japan.

We have determined the material constants of AlN, i.e., elastic stiffness, piezoelectricity and permittivity, by computer calculation from the experimental results on the SAW phase velocity and electromechanical coupling coefficient. AlN films used were single-crystal films on the basal plane of sapphire grown by metalorganic chemical vapor deposition. Until now, very few of these material constants are known. We report, for the first time, all the matrix elements of the material constants. Using the evaluated constants, it was shown that the Rayleigh wave velocity $V_{p}$ on (ZX)AIN was $5649 \mathrm{~m} / \mathrm{s}$, and the electromechanical coupling coefficient $K^{2}$ was 0.38 percent. On the other hand, $V_{p}$ on (XZ)AlN was $5675 \mathrm{~m} / \mathrm{s}$, and $K^{2}$ was 0.78 percent. The variations of $V_{p}$ and $K^{2}$ with $k H$ ( $k$ : wave number; $H$ : thickness of AlN films) on basal and $R$ planes of sapphire are in good agreements with the reported experimental results. Next, we have evaluated the SAW properties on AlN/Si grown by metalorganic chemical vapor deposition. In the (0001)AIN/(111)Si combination, where SAW propagates along the 
$[11 \overline{2}]$ direction of silicon, the dispersion curve of $V_{p}$ shows a peculiar behavior and has a cutoff, but the $K^{2}$ has the value of 0.39 percent at $k H \simeq 2.0$ (IDT: on the top surface). On the other hand, in the (YZ)AlN/(ZX)Si combination, a large value of $K^{2} \simeq 0.8$ percent is obtained at a large $k H$ value under low dispersion.

V-3 Acoustic, Optical Properties of AlN Films Grown at Low Temperature by Modified Sputter Gun,' S. ONISHI, M. ESCHWEI, S. BIELACZY, and W. C. WANG, Polytechnic Institute of New York, Route 110, Farmingdale, NY 11735.

Aluminum-nitride films have been deposited on various substrates utilizing a modified sputter gun. It provides electrical isolation between the source and the substrate allowing the use of high power density without subjecting the substrates to electron bombardment. Any electron leakage on the axis is trapped by additional magnetic field. The growing AlN film is completely free from electron bombardment. A very strong magnetic field makes it possible to sputter below $1 \mu$ Torr. This high vacuum sputtering improves $x$-ray $(002)$-peak intensity, surface smoothness, film color, transparency, and deposition rate. The deposition rate of the sputter gun is as high as $2.4 \mu \mathrm{m} / \mathrm{hr}$ at input power of $300 \mathrm{~W}$. The report will be focused on the quality of acoustic and optical properties. The insertion loss of the AIN film $(\mathrm{h} / \lambda=0.35$ ) on Corning 0211 glass is $39 \mathrm{~dB}$ at frequency of $193.4 \mathrm{MHz}$. The center to center propagation distance is $10 \mathrm{~mm}$. The design parameters of the interdigital transducer are as following: finger pair 12 , wavelength $23.3 \mu \mathrm{m}$, aperture $1.0 \mathrm{~mm}$.

${ }^{1}$ This work was partially supported by JSEP. President Peter Clarke of Sputtered Films Inc. has been most cooperative.

V-4 Characterization of ZNO/Si SAW Transducers and Resonators using Complex Return Loss Measurements, M. E. MOTAMEDI, E. J. STAPLES, and J. WISE, Rockwell International, Thousand Oaks, CA 91360 .

In this paper a method of characterizing zinc oxide/silicon delay lines and resonators in the presence of parasitic reactances is described. An automated measurement method which fits equivalent circuit models to experimental transducer return loss spectra has been developed. Using a gradient search method the correctness of the model is verified and deviations between experimental and theoretically derived return loss spectra are typically less than 5 percent. The above method has been applied to SAW delay lines on $\mathrm{ZnO} / \mathrm{Si}$ substrates as well as $\mathrm{ZnO} / \mathrm{Si}$ resonators and the experimental results will be presented. Electromechanical coupling coefficients and acoustic attenuation of $\mathrm{ZnO}$ films at high frequencies, e.g. 200-600 $\mathrm{MHz}$, have been measured using conventional single frequency measurements and will be compared to the results obtained with the gradient search method.

V-5 Design and Realization of Temperature Stable Surface Acoustic Wave Delay Lines on Silicon and Gallium Arsenide, G. CAMBON, J. LASSALE, J. M. SAUREL, and J. ATTAL, Centre d'Etudes d'Electronique des Solides, (LA 21), Université des Sciences et Techniques du Languedoc, 34060-Montpellier-Cedex, France.

In order to realize temperature stable surface acoustic wave delay lines on $\mathrm{Si}$ and $\mathrm{GaAs}$ different multilayered structures are proposed. The basic layer is $\mathrm{SiO}_{2}$. However, computer simulations show that a surface "trapping" layer is necessary in the $\mathrm{SiO}_{2} / \mathrm{GaAs}$ structure. Temperature characteristics of the $\mathrm{ZnO} / \mathrm{SiO}_{2} / \mathrm{Si}$ and $\mathrm{SiO}_{2} / \mathrm{ZnO} / \mathrm{Si}$ structures are also discussed. Experimental results show the variations of the first order temperature coefficient of phase delay versus the thicknesses of the layers. They also show up the variations of the second order temperature coefficient of phase delay versus temperature in the stability region. The temperature characteristics of these structures are comparable to that of the well-known ST-X quartz.
V.6 Temperature Coefficients of Surface Acoustic Wave Delay on GaAs, R. T. WEBSTER, Electromagnetic Sciences Division, Rome Air Development Center, Hanscom AFB, MA 01731.

A systematic search is made for orientations of GaAs having a low temperature coefficient of surface acoustic wave (SAW) delay. Experimental measurements taken on SAW oscillators operating near one $\mathrm{GHz}$ verify the results for selected cuts. The maturation of GaAs technology spurs efforts to in tegrate SAW devices and electronic devices on the same substrate. Devices using the interaction of SAW's with charge carriers in this piezoelectric semiconductor are especially exciting. The 001 cut crystal has received the most attention since it is the orientation preferred for epitaxial growth and, for SAW propagation in the 110 direction, it has the highest piezoelectric coupling. But the $+52 \mathrm{ppm}$ temperature coefficient of delay (TCD) may limit the applicability of SAW devices on this cut. Compensation of the TCD by thin film overlays is possible but lossy at higher frequencies. Moreover, a significant portion of the mechanical energy must be confined to the overlay, thereby reducing the interaction of SAW and carriers. Fabrication of active devices by ion implantation lifts the constraint of 001 orientation, allowing the possibility of a favorable trade-off between TCD and piezoelectric coupling. The data reported here gives the design engineer the basis for making this trade-off.

\section{ACOUSTOOPTIC DEVICES AND SIGNAL PROCESSING III}

\section{Poster Session}

W-1 A Computer-Based Model of Space Integrating Acoustooptic Signal Processing, ${ }^{1}$ J. P. POWERS, LCDR D. E. SMITH, LCDR M. CARMODY, and LT WELDON REGAN III, Department of Electrical Engineering, Naval Postgraduate School, Monterey, CA 93940.

This paper presents a computer based model of general purpose signal processors that use an acoustooptic (Bragg) cell for spatially forming the spectrum of an RF signal (i.e., spatially integrating processors). The model includes the ability to represent: (1) arbitrary transfer functions for the acoustic transducer; (2) arbitrary beam profile for the incident laser beam; (3) diffraction effects as the light forms the spectrum; (4) diffraction inefficiency due to a multifrequency acoustic signal. The model has been used to predict expected sidelobe levels for various input laser beam profiles and can be used to predict trade-offs between spot size (and hence frequency resolutions) versus sidelobe levels. Additionally the model has been useful in an investigation of the sensitivity of optical excision processors to the placement of the excisor. It has also been used pedagogically to demonstrate many of the transient effects expected with acou stooptic processing of short RF pulses.

\section{${ }^{1}$ This work was partially sponsored by the Naval Electronics Systems Command.}

W-2 Dual Differential Interferometer for Measurements of Broadband Surface Acoustic Waves, T. M. TURNER and R. O. CLAUS, Department of Electrical Engineering, Virginia Poly technic Institute and State University, Blacksburg, VA 24061.

A simple dual interferometer which uses two pairs of orthogonally polarized optical beams to measure both the amplitude and direction of propagation of broadband ultrasonic surface waves is described. Each pair of focused laser probe beams is used in a separate wideband differential interferometer to independently detect the component of surface wave motion along one direction on the surface ${ }^{1,2}$ By combining the two output signals corresponding to both components, the two-dimensional surface profile and its variation as a function of time may be determined. Although the system has an optically adjustable $-3 \mathrm{~dB}$ acoustic bandwidth of more than two decades (e.g., $30 \mathrm{kHz}$ to 
$3 \mathrm{MHz}$ for acoustic emission measu rements) and can detect peak displacements in the subAngstrom range, it is insensitive to low frequency specimen translations. Potential applications in nondestructive evaluation are described. ${ }^{3}$

' C. H. Palmer, R. O. Claus and S. E. Fick, Appl. Opt., vol. 16, p. 1849 (1977).

${ }^{2}$ R. O. Claus and J. H. Cantrell, Proc. Ultrason. Intl., Brighton, UK, June 1981 .

${ }^{3}$ This work was supported by NSF and NASA.

†W-3 Large Angular Aperture CDSE Tunable Acoustoop tic Filter, I. C. CHANG and P. KATZKA, Applied Technology, Sunnyvale, CA 94086

The angular aperture of a tunable acoustooptic filter (TAOF) is inversely proportional to the square root of the birefringence of the acoustooptic material, while the spectral resolution is inversely proportional to the resolution factor $b, b=2 \pi\left[\Delta n+\nu_{0}\left(\partial / \partial \nu_{0}\right)\right]$, where $\Delta n$ is the birefringence and $\nu_{0}=1 / \lambda_{0}$. In the short wavelength band edge region, the birefringence of CdSe goes through zero, while the second term in the resolution factor becomes dominant. In the vicinity of the birefringence cross-over region, the angular aperture of a CdSe TAOF increases substantially. A noncollinear CdSe TAOF has been designed, $u$ tilizing band edge enhancement of the field of view. The device achieves $5.2 \AA$ spectral resolution at $6328 \AA$ with a 59 degree external field of view.

W-4 A Monolithic ZnO/Si PI-DMOS SAW Convolver, ${ }^{1}$ A. E. COMER and R. S. MULLER, Department of EECS and the Electronics Research Laboratory, University of California, Berkeley, CA 94720.

A new Si SAW convolver, fabricated compatibly with a Si planar DMOS transistor, has been built and tested. In contrast to previous $\mathrm{Si}$ convolvers, this structure employs an active device (high-speed piezoelectric DMOS transistor) to perform the mixing and integration functions for the convolution. The structure consists of two IDT's, formed on five micron-thick sputtered $\mathrm{ZnO}$ films, on either end of a waveguiding signal path. Two $45 \mathrm{MHz}$ signals are guided along the width of the one mm-wide PI-DMOS transistor which is biased into the currentsaturated mode of operation. In this nonoptimized prototype structure the $\mathrm{ZnO}$ coupling coefficient is only 15 percent of previously achieved values which results in $16 \mathrm{db}$ loss of signal power. A bilinear conversion efficiency $F_{T}$ of $-70 \mathrm{dbm}$ is measured when the convolution output at the transistor drain feeds a 50-ohm transmission line. With a one $\mathrm{cm}$ wide DMOST and higher quality $\mathrm{ZnO}$ films, $F_{T} s$ as high as $-34 \mathrm{dbm}$ can be expected.

${ }^{1}$ This work supported in part by AFOSR Contract (ISEP)F49620 79-C-0178, and in part by a grant from the Rockwell International Science Center, Thousand Oaks, CA.

\section{BULK WAVE EFFECTS AND DEVICES}

Poster Session

X-1 [Invited] High Overtone Bulk Resonator Stabilized Microwave Sources, R. A. MOORE and J. T. HAYNES, Westinghouse DESC, Baltimore, MD 21203 ; B. R. McAVOY, Westinghouse R\&D Center, Pittsburgh, PA 15235.

Several new techniques, dielectric resonators, SAW resonators, skimming mode bulk acoustic resonators and high overtone bulk acoustic wave (BAW) resonators are being advanced for low noise stabilization of microwave signal sources. This paper will provide a comparison of properties and potentials for application for each of these resonator types. It will also dwell in depth on the high overtone BAW stabilized signal source. It is the use of film transducers, obviating the need for self-piezoelectric coupling which allows high overtone operation. This makes available a greater variety of crystal materials and cuts for resonator fabrication leading to higher $Q s$, range of operating frequency and more closely spaced resonant frequencies. $Q s$ to 20,000 have been measured. Double these values are projected by independent acoustic attenuation measurements. Resonance periodicities are important for frequency agile sources, from less than five $\mathrm{MHz}$ to greater than 100 MHz separation have been demonstrated. Though temperature stable cuts of high $Q$ medium have not been tested on high overtone resonators, a range of shear cuts in $\mathrm{LiNiO}_{3}$ has been identified which promises temperature stable operation. The paper will also cover progress toward mechanization in ruggedized airborne oscillators for both low noise operation and greater frequency agility. Specific circuits examples will be described and test results discussed.

\section{X-2 The Excitation of Coupled Thickness Shear-Flexural Modes in Rotated $Y$-Cut $\mathrm{LiTaO}_{3}$ Plates, J. MURPHY, Westinghouse Research} Center, Pittsburgh, PA 15235.

Following the procedure given by Mindlin and Deresiewicz ${ }^{1}$ for AT cut quartz plates, the electrode shape required to excite a single flexural overtone coupled to the fundamental shear mode of $\mathrm{LiTaO}_{3}$ has been determined. The results of experiments on 1628 rotated $\mathrm{Y}$ cut $\mathrm{LiTaO}_{3}$ plates with the theoretically determined electrode configuration will be presented.

${ }^{1}$ R. D. Mindlin and H. Deresiewicz, J. Appl. Phys., vol. 25, p. 25, 1954.

X-3 Deep-Bulk-Acoustic-Wave (DBAW) Devices Utilizing Interdigital Transducers, C. N. HELMICK, JR., and D. J. WHITE, Naval Weapons Center (Code 3814), China Lake, CA 93555; and K. M. LAKIN, Ames Laboratory, Iowa State University, Ames, IA 50011.

Interdigital transducers (IDT's) have long been employed for launching and receiving surface-acoustic waves (SAW's) and, more recently, shallow bulk-acoustic waves (SBAW's) traveling along a surface of a piezoelectric crystal. We now report on the use of IDT's located on different faces of a crystal to launch and receive acoustic waves traversing the bulk material, which we refer to as DBAW's (deep bulkacoustic waves). Specifically, we consider the case where the IDT's are placed on opposite faces of a quartz $X$-axis. Phase matching leads to the condition $f_{0} \lambda_{0}=v / \cos \alpha$, where $f_{0}=$ center frequency, $\lambda_{0}=$ IDT period, $v=$ wave velocity, and $\alpha=$ inclination angle of the wave vector. The limit as $\alpha \rightarrow 0$ yields a low-frequency cutoff, corresponding to the SBAW configuration (IDT's on the same surface). The DBAW configuration (IDT's on different surfaces) yields an operating frequency $(1 / \cos \alpha)$ times that of a SBAW device employing the same transducer design. One must, of course, take into account the anisotropy of velocity, beam steering, and coupling coefficient. In addition to enabling a higher operating frequency, a DBAW device might be designed to utilize the angular dispersion with frequency (e.g., with multiple output transducers) or possibly to utilize beam steering for obtaining (nearly) perpendicular energy transmission. Preliminary observations of a few quartz DBAW devices will be presented and discussed.

X-4 Some Properties of Electroded Piezoelectrics Realized and Illustrated in AT-Cut Quartz, JOHN H. SHERMAN, JR., Consultant, 2022 Woodcrest Drive, Lynchburg, VA 24503.

Ballato and Lukaszek have offered a novel equivalent circuit for electroded quartz resonators with unbounded electrodes, in which the only approximation is the assumption that the electrodes could always be treated as conductive mass of zero or negligible thickness. The quartz body is modeled as a transmission line. This model was generalized by Ballato into the basis of a volume of tables issued as an ECOM Technical Report receiving only limited distribution. This paper recomputes 
tables for AT-cut quartz in double precision in order to examine a number of relationships of interest to the designer of resonators. Resonant and antiresonant frequencies and ratios of frequencies, also capacitance ratios and ratios of capacitance ratios, are tabulated for mass loads up to five percent through the ninth harmonic mode. It is shown that a number of relationships found to be uniform in practice, but not anticipated in the first order resonator theory used as the basis of most resonator design algorithms, are predicted by this higher order theory. The paper also contains a FORTRAN routine to compute the frequency lowerings to assist similar studies of other cuts or materials.

X-5 Forced Thickness-Extensional Trapped Energy Vibrations of Piezoelectric Plates, D. V. SHICK, H. F. TIERSTEN, and B. K. SINHA, Rensselaer Poly technic Institute, Troy, NY 12181.

An analysis of PZT-7A plates driven into thickness-extensional trapped energy vibrations by the application of a voltage to strip electrodes is performed. The plate waves in the unelectroded region that carry energy away from the trapped mode are included in the treatment. All other treatments of thickness-extensional trapped energy modes in piezoelectric plates have ignored the radiating plate waves, which enabled the consideration of an eigenvalue problem rather than the inhomogeneous forced vibration problem considered here. The solution consists of a sum of the driven thickness solution and the pertinent plate eigensolutions for shorted electrodes in the electroded region and for zero surface charge in the unelectroded region. Since the sum of the solutions in each region satisfies the differential equations and boundary conditions on the major surfaces exactly, the substitution in to the appropriate variational principle of linear piezoelectricity yields integral conditions across the thickness of the plate at the edge of the electrodes. The resulting system of inhomogeneous linear algebraic equations yields the response.

${ }^{1}$ Schlumberger-Doll Research Center, Ridgefield, CT 06877.

\section{MEDICAL ULTRASOUND}

\section{Poster Session}

Y-1 Chemical Dosimetry of Ultrasonic Cavitation, K. S. SUSLICK, P. F. SCHUBERT, and J. GAWIENOWSKI, School of Chemical Sciences, University of Illinois, $505 \mathrm{~S}$. Mathews Ave., Urbana, IL 61801.

We have utilized several new chemical dosimeters to evaluate the efficacy of various solvents in producing cavitational sonochemistry. Aqueous and alcoholic solvent systems are easily standardized using the oxidation of $I^{-}$to $I_{3}^{-}$by spectrophotometry. The more common addition of $\mathrm{CCl}_{4}$ in order to increase rates of $I_{3}^{-}$production dramatically reduces the reproducibility of this system, which in our hands can be well within 5 percent. The use of the stable free radical, diphenylpicrylhydrazyl, as a radical trap in nonaqueous sonochemistry will be discussed. The relative rates of radical production decreases in the following order: methanol, decane, cyclohexane, acetonitrile, acetone, toluene, and benzene. A third dosimeter, the decomposition of ferrocene, has proved useful as the measure of the intrinsic ability of various solvents to support sonochemical reations.

Y.2 A Method of Noninvasive Determination of Inhomogeneous Layer's Structure and Adaptive Image Reconstruction, T. SATO, M. HIRAMA, and O. IKEDA, The Graduate School, Tokyo Institute of Technology, 4259, Nagatsuta, Midori-ku, Yokohama-shi, 227 Japan.

The complex amplitude distribution of an inhomogeneous layer between the array and object is determined noninvasively by only repeating the transmission and detection by the array. It is carried out in an optimum way by using the degree of focusing as the measure, that is, by measuring the degree of correlation between the transmitted signals and the detected signals which are reflected from the object. A concrete algorithms of iterative procedure for the estimation is given. This method is examined by several numerical analyses under considerable conditions and its usefulness is shown. This method is implemented in an actual ultrasonic array imaging system and the effects as an adaptive imaging system are also assured by several experiments carried out under fairly severe inhomogenuities.

†Y-3 Time Varying Filtering Properties of Focused and Nonfocused Transducers due to Diffraction and to Frequency Dependent Attenuation, M. FINK, D. NEGRERA, Laboratoire de Mécanique Physique, Université, Paris VI, France.

This paper is devoted to a theoretical analysis of the time varying filtering response of both plane piston and concave spherical transducer. The near field low pass filtering effect due to diffraction will be emphasized; its particular dependence in the case of plane and focused transducers is discussed. The time varying filtering response linked to frequency dependent attenuation is compared to the diffraction filtering effect. Simple transducer calibration curves are presented for different frequency dependent attenuations. One of the most interesting calibration curves is the one that shows the mean frequency of the echographic response versus depth. It will be shown that the "near field" concept is strongly dependent on the value of the attenuation slope versus frequency. Time varying filtering corrections needed as well in the attenuation slope estimation technique, as in the pulsed Doppler technique will be discussed.

†Y-4 Image Degradations in Ultrasonic CT, T. ITOH and J. S. CHOI, Research Center, Aloka Company, Ltd., Mitaka 181, Japan; and M. NIKAJIMA, Department of Electrical Engineering, Keio University, Yokohama 223, Japan.

An experimental data acquisition system for transmissive ultrasonic $\mathrm{CT}$ has been developed. The system reconstructs a velocity distribution and two attenuation constant distributions (one is reconstructed from the maximum amplitude and the other from the integrated amplitude signals after full-wave rectification). The scanning time for $\mathbf{4 5}$ views by 128 samples is about five minutes. A pair of $3.5 \mathrm{MHz}$ PZT transducers is used and the accuracy of propagation time and the dynamic range are $10 \mathrm{~ns}$ and $100 \mathrm{~dB}$, respectively. Though we obtained considerably good results in clinical use, the reconstructed images were degraded by reflection, refraction, etc. We also studied the amounts of image degradations by experiments using phantoms and computer simulation. Phantoms are made of water-based gel, whose velocity and attenuation are controlled by mixing graphite powder and saccharose, respectively. In the results, it is obtained that the degradations are little when the phantom velocity differs slightly (about $10 \mathrm{~m} / \mathrm{s}$ ) from surrounding medium such as water. However it is necessary to correct the influences of reflection or refraction for such cases that the velocity difference is not small. We will discuss about the correction.

Y-5 A $25 \mathrm{MHz}$ Digital Acquisition System and Applications to Diagnostic Ultrasound, ${ }^{1}$ P. L. CARSON ${ }^{2}$, G. A. THIEME, R. A. BANJAVIC, and C. R. MEYER ${ }^{2}$, University of Colorado Medical School, Denver, CO 80262; and J. J. KRASOVEC, Precision Data Systems, Littleton, CO 80120 .

A $25 \mathrm{mps}$ digital data acquisition system with sampling controls designed for ultrasound applications is described. Eight-bit samples are acquired and stored in $8 K$ bytes of memory under microprocessor control. The digitizer is linked to a general laboratory computer both by a 16-bit parallel interface for rapid transfer of sample data and by a standard RS232 interface shared with a standard video terminal for sending operational control codes and receiving serially transferred 
sample data (optional). New test methods demonstrating system analog-to-digital conversion accuracy are presented. Interfacing of the system to a compound $B$-mode scanner is described, and $B$-mode images with corresponding digitized RF waveforms are presented for a tissue-equivalent phantom.

1 This work was supported in part by a PHS Grant RO1-CA-25323 awarded by the National Cancer Institute, DHHS, and by a contract from the National Electrical Manufacturer's Association.

${ }^{2}$ Present address: Univ. of Michigan, Department of Radiology, Ann Arbor, MI 48109

Y-6 Rational-Gain-Compensation for Attenuation in Ultrasonic Cardiac Imaging, ${ }^{1}$ H. E. MELTON, JR., and D. J. SKORTON, Departments of Electrical Engineering and Medicine, University of lowa and Iowa City Veterans Administration Medical Center, Iowa City, IA 52242.

The first step toward direct, reliable detection of myocardial infarction in vivo is a rational accounting for attenuation present between the transducer and sites of interest within the heart. One method was tested which compensated for attenuation on a line-by-line basis in real-time two-dimensional ultrasonic scans. This method dpends on the fact that ultrasonic scatter from blood is much smaller (absent) than scatter from myocardium, whether normal or abnormal. When backscatter was "absent" receiver gain was increased at a rate suitable to compensate for attenuation by blood; when backscatter was "present" receiver gain was changed at a rate needed to compensate for attenuation by myocardium. By using this method for compensating for attenuation (rational-gain-compensation, RGC) instead of the commonly used depth-gain-compensation, widely separated regions of myocardium which were nominally identical were depicted in scans with nominally the same attributes.

${ }^{2}$ This research was supported in part by the following grants: Veterans Administration Research Advisory Group Award and Advanced Technology Laboratories.

+Y-7 Sinuscan, A Portable Ultrasonic Unit to Detect Maxillary Sinusitis, M. LUUKKALA and P. MATTILA, Department of Physics, University of Helsinki, Helsinki, Finland; and M. REVONTA, Kanta-Hämeen Keskussairaala, Hämeenlinna, Finland.

The diagnosis of maxillary sinusitis in humans is usually done either by punctuation, which is an invasive and unpleasant method, or by $x$ ray radiography, which is an ionizing method. Maxillary sinusitis is an inflammation of the sinus cavities. An extended period of thinitis (flu) may cause collection of secretion in the cavities which may be detected by ultrasound. We have constructed a small, lightweight ultrasonic unit called Sinuscan, which is specifically adapted to detect maxillary sinusitis. The ultrasonic unit has been thoroughly tested in hospital surroundings and the results obtained were always compared with puncture and radiography. It turned out that the confidence of Sinuscan was better than 80 percent and at least as good as radiographic method.

\section{LOW LOSS SAW FILTERS}

\section{Chairperson: R. S. KAGIWADA}

Z-1 Surface Wave Devices using Low Loss Filter Technologies, D. C. MALOCHA, Sawtek, Inc., 2541 Shader Road, Orlando, FL 32854.

Low loss filters are being accepted as viable solutions for filter functions where low loss, minimum passband amplitude ripple and low group delay distortion are required. The primary transducer configurations of interest are the three transducer approach, the group type unidirectional transducer (UDT), and the three-phase and quadrature three-phase (or pseudo four-phase) UDT. The various approaches each have advantages and disadvantages in fabrication and filter performance which normally define their usage. This paper will compare the various design criteria for choice of the proper structure, evaluate the performance of each and present experimental results for verification. In order to abtain optimum filter performance, tight matching to the load using high $Q$ components is normally required. This condition provides an accurate means for obtaining the thin film resistive loss without precise measurement of impedances by using the metal's temperature coefficient of resistance. An analysis and experimental data is provided for predicting the resistive loss and the insertion loss variation over temperature for a three-phase low loss filter.

Z-2 A Comparison of Feeding Techniques for Group-Type Unidirectional Transducers, ${ }^{1}$ R. L. MILLER and B. J. HUNSINGER, Coordinated Science Laboratory, University of Illinois, Urbana, IL 61801.

A group-type unidirectional transducer (G-UDT) has two electrical ports which must be fed in phase quadrature at the center frequency. Previous feeding schemes have used either a lumped-constant network, or at most a quarter period of delay using a cable, thus showing only a narrow-band cancellation of the reverse wave. It is shown that, by matching the electrical delay to the acoustic delay between the two transducers within each group, it is possible to either optimize the forward or reverse wave. For example, using this delay and an additional $\pi$ radians phase shift it is possible to substantially cancel the reverse wave over a broad band of frequencies, significantly improving the forward/reverse ratio of the device. Another scheme is proposed in which an amplifier is used to each electrical port. The nonreciprocal properties of the amplifiers isolate the two electrical ports from each other, causing important differences in device operation. This will be shown to be useful for applications like two-port SAW resonators. Finally, the reflection properties of G-UDT's using all of these feeding schemes will be compared.

1 This work was supported in part by the Joint Services Electronic Program (U.S. Army, U.S. Navy, U.S. Air Force) under Contract N00014-79-C-0424) and by the Rome Air Development Center, Deputy for Electronic Technology, under Air Force Contract F19628-81-K-0031.

2-3 Low Loss Ultra Flat SAW Filters using Group-Type Unidirectional Transducers, W. S. ISHAK, Hewlett-Packard Laboratories, Palo Alto, CA 94304.

Low loss ultra flat surface acoustic wave (SAW) filters using grouptype unidirectional transducers on $\mathrm{LiNbO}_{3}$ are investigated. Filters are tested at center frequencies of $360,650,725 \mathrm{MHz}$ with fractional bandwidth of about 3 percent. Two different $\mathrm{LiNbO}_{3}$ cuts are used: $Y-Z$ cut and $128^{\circ}$-rotated $Y_{\text {-cut; }}$ the differences in filter characteristics for both cuts are examined. Proper weighting of the group-type transducers resulted in insertion loss as low as $5.3 \mathrm{~dB}$, out-of-band rejection of more than $40 \mathrm{~dB}$ and very low amplitude ripples (as low as 0.005 dB). Two apodization functions are used and comparison between results is given. The ripples in the group delay are $<20 \mathrm{~ns}$. The filters were fabricated using photolift-off technique, mounted in T0-66 headers and matched using single air-wound coils per transducer. An impulse model is used to synthesize the transducers and calculate the values of the tuning elements for matching and phase shifting. Very good agreement between model and experiment is obtained. ${ }^{1}$

${ }^{1}$ Yamanouchi, et al., Ultrasonics Symp. Proc. IEEE, pp. 317-321, Sept. 1975

2-4 Group-Type Unidirectional Transducers at $L$-Band Frequencies Utilizing Withdrawal Weighting, B. R. POTTER, Texas Instruments Incorporated, MS 255, P.O. Box 226015, Dallas, TX 75266.

Many systems require high quality filters in the $L$-band frequency range. SAW filters meet many of the stringent specifications for IF 
filters in the 1 to $2 \mathrm{GHz}$ frequency band. Reported in this paper are SAW filters at $1227.6,1575.42$, and $900 \mathrm{MHz}$. These $L$-band filters utilize both apodized and withdrawal weighted transducers to achieve greater than $-55 \mathrm{~dB}$ frequency sidelobes. Withdrawal weighting and a unique apodization method for group-type unidirectional transducers are presented along with insertion loss and phase data for the two higher frequency devices. For example, the $900 \mathrm{MHz}$ devices had only 6-7 dB insertion loss even though they were constructed on ST-cut quartz. The transducer technology for $L$-band filters will be discussed in detail along with the GPS application.

Z-5 Low Loss SAW Bandpass Filters, ${ }^{1}$ P. DEFRANOULD and J. DESBOIS, Thomson-CSF DASM Cagnes/Mer-06802, France.

Unidirectional three phase technology transducers, first proposed by Texas Instruments was developed both on YZ- $\mathrm{LiNbO}_{3}$ and on STQuartz. We proposed a new theoretical approach based on a so called "mixed matrix" model which furthermore takes into account the losses in dielectric crossovers and in matching networks. Theoretical results showed good agreement with measured responses and confirm that the parasitic losses are very critical on ST-Quartz but much less on $\mathrm{LiNbO}_{3}$. So, by using simple technology, total insertion loss of 2 to $4 \mathrm{~dB}$ was measured for two port delay lines realized in 100-200 $\mathrm{MHz}$ frequency range. We also demonstrate operation at second harmonic which allowed higher frequency range for fundamental transducer pattern. A filter using a finger withdrawal weighting technique was realized on ST-Quartz. The measured insertion was $4 \mathrm{~dB}$ at a center frequency of $105 \mathrm{MHz}$, the ripples in the bandwidth are $\pm 0.1 \mathrm{~dB}$ and out of band lobes rejection is $-50 \mathrm{~dB}$.

'This work has been sponsored by DRET.

2-6 Low-Loss Low Spurious Reflective Dot Array Filter using ThreePhase Transducers, D. PENUNURI and J. L. THOSS, Hughes Aircraft Co., Fullerton, CA 92634.

In this paper we describe a new device in which a reflective dot array having extremely low acoustic insertion loss is combined with a set of three-phase input and output transducers. The resulting filter exhibits an insertion loss of less than $10 \mathrm{~dB}$ with very low spurious levels. The three-phase transducers are fabricated using electron beam evaporated dielectric films as crossover insulators. A simple lift-off process is employed to pattern the dielectric film on the device. The dielectric crossover film is retained in order to improve the structural integrity of the device. Unidirectional transduction is achieved by tuning a simple phase matching network derived from a cross field circuit model generalized to include crossover capacitance and electrode resistance. The reflective dot array is designed to give both matched narrow band filter response and very low loss while minimizing multiple reflection distortions inherent in high performance stripe array filters. To obtain low loss, both aluminum and gold metalized dots are examined. The desired filter selectivity and waveform matching is attained by appropriately weighting the reflective arrays.

\section{NDE STRESS MEASUREMENTS}

Chairperson: G. S. KINO

AA-1 Stress Characterization by Combined Time-of-Flight and Attenuation Measurements of Polarized Shear Waves, E. SCHNEIDER, K. GOEBBELS, and G. HÜBSCHEN, Fraunhofer-Institut für zerstörungsfreie Prüfverfahren, D-6600 Saarbrücken, FRG.

The physical background of stress measurements by ultrasonic methods is well known for a long time, but the practical realization has turned out to be difficult due to two reasons: residual stress changes the sound velocity $v$ only by a small amount, so that precise measurements of $v$ require accurate measurements of time-of-flight and ultrasonic path length. Texture and microcsopic structure also influence the sound velocity. Referring to the first problem, two shear waves polarized perpendicular to each other and propagating along the same path, enable the determination of residual stress quantitatively without thickness measurements. In order to separate the influence of stress and texture on the sound velocity, the sound attenuation has been measured additionally as a function of the direction of polarization. The absorption changes in a characteristic way, if there exist residual stresses in the sample whereas it is nearly constant in the case of texture. For the generation of sound both piezoelectric- and electromagnetic-acoustic transducers (EMAT's) were used. The EMAT transducers allow the generation of linearly polarized transverse waves with a high degree of polarization.

AA-2 Determination of Material Stress from the Temperature Dependence of the Acoustic Natural Velocity, ENGMIN J. CHERN ${ }^{1}$ and JOSEPH S. HEYMAN, NASA Langley Research Center, Hampton, VA 23665 .

Theoretical analysis has shown that material anharmonic properties are strongly coupled to material stress state. In this paper, a specific theory is presented along with experimental verification that the temperature dependence of natural velocity (thermal acoustic constant) can be used to determine changes in stress in isotropic materials. Data will be presented for both pulsed phase locked loop and leading edge time-of-flight ultrasonic velocity measuring techniques. Tests were performed using $2.25 \mathrm{MHz}$ longitudinal waves in axially stressed rods of polycrystalline aluminum over the temperature range $25^{\circ} \mathrm{C}-65^{\circ} \mathrm{C}$ and the stress range 0-175 MPa. The data show a linear relationship with a greater than 6 percent change in thermal acoustic constant with respect to stress up to the tested value of $175 \mathrm{MPa}$. A comparison of the present data with previous results obtained by Salama and Ling suggests the potential application of this technique to measurements of material stress.

'Research Associate, Department of Physics, College of William and Mary, Williamsburg, VA 23185.

AA-3 Measurement of the Three-Dimensional Variation of Stress, ${ }^{1}$ S. BENNETT, D. HUSSON, and G. S. KINO, Ginzton Laboratory, Stanford University, Stanford, CA 94305.

The velocity of an acoustic wave passing through a solid changes linearly with the applied stress. Now, for the first time, we have made a measurement of the three-dimensional variation of stress. The system operates in transmission using two beams, focused beam from a hollow spherical transducer coaxial with an unfocused beam from a plane piston transducer. The phase difference between the signals received at similar transducers has been shown theoretically to depend mainly on the stress field in the neighborhood of the focal region. Furthermore, when longitudinal waves are employed, the component of stress of importance is that in the direction of propagation. By using this technique with a scanned two-part transducer, we have been able to measure the stress field variation around a crack and obtain good agreement with theoretical predictions of the stress variation. A second experiment conducted on a sample of 304 stainless steel stressed into the yield range, shows the effect of plasticity and a maximum stress at some distance from the crack.

${ }^{1}$ This work was supported by the Electric Power Research Institute under Contract RP609-1.

†AA-4 A Possible Ultrasonic Residual Stress Standard, G. V. BLESSING, N. H. HSU, and T. M. PROCTOR, JR., National Bureau of Standards, Washington, DC 20234. 
The need for a reliable technique to nondestructively evaluate the residual stress state of a material is widely recognized. While ultrasonic elasticity or velocity measurements are frequently applied to such an evaluation, meaningful measurements are very difficult. A significant part of the difficulty is the lack of a sample with a known stress state, especially for a zero residual stress reference velocity $V_{0}$. We present the initial results of one approach to develop such a standard sample that would provide an absolute relationship between stress and velocity. The approach is to shrink fit a material to a known stress level, observing the change in elasticity. An aluminum sample bar was measured for its relative elasticity and scattering properties. A region possessing uniform properties was selected for ring and plug sections that would be subsequently used to construct a shrink-fit disk assembly. Aluminum was chosen for its relatively large stress-acoustic constant. Longitudinal and shear wave time-of-flight measurements were made before and after a) the sample sectioning and b) the disk assembly. The experimental results are compared with a theoretical analysis that relates the velocity and stress profiles.

AA-5 Computer Controlled Ultrasonic Motion Measurements, M. A. HAMED, J. R. DICKERSON, and W. F. RANSON, College of Engineering, University of South Carolina, Columbia, SC 29208.

Modern applications of ultrasonic NDE have involved the detection and display of defects in solids. However, the presence of a crack or void can significantly alter structural integrity, therefore, measurements of internal boundaries which describe deformation should be coupled with NDE measurements for critical evaluation of crack like defects. A computer based pulse-echo system which determines displacements of a subsurface scattering layer is described in the following manner: a pulse-echo scan is made of a scattering surface over some area which represents a undeformed configuration. After the surface is displaced a pulse-echo scan is made over the same area which describes the deformed configuration. Displacement components of the scattering surface are calculated by assuming that a small region of the undeformed configuration is mapped by a linear expansion of the displacement function to a small region of the deformed configuration. Thus, a small region of the deformed configuration is calculated with the reference as a measure of object motion. The method is illustrated by several examples which describes the effect of finite strains on the calculation measurements.

AA-6 Detection of Acoustic Emission in Composites by Optical Fiber Interferometry, J. C. WADE, P. S. ZERWEKH, and R. O. CLAUS, Department of Electrical Engineering, Virginia Polytechnic Institute and State University, Blacksburg, VA 24061.

Acoustic emission in prestressed composite panels have been detected using embedded single mode optical fibers. Static loading of the composite matrix produces acoustic emission events, pulsed ultrasonic waves which mechanically modulate the embedded fiber geometry and phase modulate the transmitted optical field. This modulation is detected by optical interferometry and Fourier optical processing techniques ${ }^{1}$ to produce an electronic signal proportional to acoustic field amplitude integrated along the length of the fiber in the specimen. ${ }^{2}$ Experimental static and dynamic calibration of the detection system is discussed and frequency domain characterization of the acoustic field interaction with the fiber is presented. Potential applications in the nondestructive analysis of structural composites are suggested.

\footnotetext{
${ }^{1}$ R. O. Claus and J. H. Cantrell, Proc. IEEE Ultrasonics Symposium, Boston, MA, Nov. 1980.

${ }^{2}$ P. S. Zerwikh and R. O. Claus, Proc. IEEE Region 3 Conf., Huntsville, $\mathrm{AL}$, April 1981.

${ }^{3}$ This work was supported by NASA Grant NAG-1-68.
}

\section{TISSUE CHARACTERIZATION-ACOUSTIC MICROSCOPY}

\author{
Chairperson: S. A. GOSS
}

BB-1 The Research Characteristic Echostructures of the Different Component of Mammary Tissue, J. L. LAMARQUE, M. J. RODIERE, J. ATTAL, A. DJOUKHADAR, and E. BOUBALS, Service de radiodiagnostic, Hopital Saint-Eloi 34059 Montpellier-Cedex-France and Université des sciences et techniques du Languedoc Montpellier.

The goal of the study is to find at a microscopic level the characteristical echostructures of tissue and ultrasounds variations according to histology or pathology. We have studied on histological sections the pictures obtained by acoustic microscope and their variability according to the ultrasound beam (reflection or transmission), the thickness of the studied sections, the hydratation of the studied tissue. In acoustic microscopy mammary tissues are altered only by the procedures of fixation (no staining). All the images were obtained with an acoustic frequency of $600 \mathrm{MHz}$. As acoustic microscope on elastic properties of the cells that are examines; differences between the elastic properties of normal or abnormal cells can be visualized. Samples are then stained and studied in light microscopy and correlations are performed. We are trying to relate this microscopic echostructures to macroscopy in vivo in the aim of giving to physicians and echographists reliable and characteristic signs of a tissue and its pathology because we think that it is essential to understand the obtained pictures in mammary ultrasonography and in establishing the correct echography diagnosis. The first results are shown, discussed, and correlated with historadiography, mammography, and computed tomography.

BB-2 Phase Mapping in Acoustic Microscopy, F. S. VINSON and R. C. EGGLETON, Ultrasound Research Division of the Indianapolis Center for Advanced Research, Indianapolis, IN 46202.

A new means has been developed by which phase data can be mapped onto acoustic micrographs obtained using a $100 \mathrm{MHz}$ scanning laser acoustic microscope (Sonoscan, Inc., Bensenville, IL) such that quantitative measures of local variations in specimen elasticity can be examined. First, phase contour lines produced by a phase-detecting circuit are superimposed on the micrographs. The micrographs are then digitized, and an image processor (IIS, Model 70E, Stanford Tech., Sunnyvale, CA) is used to locate the positions of the phase lines. Images are then produced in which the amplitude of the transmitted ultrasound is represented by intensity and the phase by color. In studying materials in which a single mode of sound transmission is known to be predominant, as, for example, in biological soft tissues, the phase map can be converted directly to an acoustic phase velocity map. This is particularly useful for tissue characterization and nondestructive evaluation purposes, in that regional variations in elasticity can be ascertained. The phase mapping technique has been successfully applied to a study of the anisotropic elastic properties of soft tissues, and it is anticipated that new information concerning the mechanisms of sound transmission in materials will become available using the technique.

BB-3 Tissue Anisotropy Examined Using the Acoustic Microscope, F. S. VINSON and R. C. EGGLETON, UItrasound Research Division of the Indianapolis Center for Advanced Research, Indianapolis, IN 46202.

It has been known for some time that, in many biological soft tissues, the speed of ultrasound propagation is dependent on the direction of propagation. This phenomenon has been observed in scanning laser acoustic microscopy, in spite of a rather narrow range of available angles of propagation. While linear anisotropic elastic theory has been applied to characterize ultrasonic interactions in industrial materials and in bone, this approach, to the best of the authors' knowledge, has not been applied to biological soft tissues. In the current study, a 
phase mapping acoustic microscopic technique was used to measure speed of sound values as a function of the direction of propagation in seven frog sartorius muscles and three rat tail tendon fiber specimens. A least squares approach was then used to determine the values of the anisotropic elastic constants, based upon a linear anisotropic elastic model, which most accurately described the data. A consistent relationship among the elastic constants was observed for all of the specimens and, in all cases, a better fit of the data was obtained using the anisotropic model as opposed to the isotropic model.

BB-4 Examination of Arterial Tissue Using $100 \mathrm{MHz}$ Acoustic microscopy, ${ }^{1}$ W. D. McNEELY and S. A. GOSS, Indianapolis Center for Advanced Research, Inc., Ultrasound Research Division, 410 Beauty Avenue, Indianapolis, IN 46202.

An acoustic microscopic study of human arterial tissues using a 100 MHz scanning laser acoustic microscope (Sonoscan, Inc.) was undertaken to visualize structural layers in the vessel wall, and to determine the ultrasonic velocity associated with each layer. Variation in the acoustic properties of each layer (intima, media, internal elastic mambrane) was expected, since each contains varying amounts of connective tissues, fat, and other components previously shown to influence acoustic propagation in biological media. Results in tissues excised from the external iliac artery caudal to the bifurcation and examined in radial cross section indicate that (1) the intima, media, internal elastic membrane, and fat deposits can be visualized; (2) that the visualizability of such structural layers appears to be dependent on the age of the individual; (3) acoustic velocity in elastic structures such as the internal elastic membrane are greater than that of the surrounding tissue; and (4) velocity variation patterns across the vessel wall appear to change with age, presumably associated with an increase in vessel inelasticity, with young individuals exhibiting few, and older individuals many, variations overall.

${ }^{1}$ This work was supported in part by the Indianapolis Center for Advanced Research, Inc.

BB-5 Liver-Tissue Characterization by Digital Spectrum and Cepstrum Analysis, ${ }^{1}$ F. LIZZI ${ }^{2}$, D. KING ${ }^{3}$, E. FELEPPA ${ }^{2}$, N. JAREMKO ${ }^{2}$, P. $\mathrm{WAI}^{3}$, Riverside Research Institute, New York, NY 10023; Columbia University College of Physicians and Surgeons, New York, NY 10032.

In vivo liver is being studied using a digital system for clinical data acquisition and processing. This system interfaces a high quality real time scanner with a PDP $11 / 55$ computer. The clinician utilizes the real time image to identify and locate a tissue region of interest. RF echo signals from the region of interest then are digitized and stored for subsequent computer analysis. Spectral and cepstral processing have proven to be the most useful methods. In normal livers, cepstra typically show a tissue-structure dimension of approximately $0.8 \mathrm{~mm}$, which corresponds to the liver lobule size and may be a manifestation of the normal spacing between triads. Liver abnormalities involving diffuse disease, such as cirrhosis, are expected to cause cepstra to show a triad-spacing dimension outside the normal range.

1 This work is supported, in part, by PHS/NIH Grant RO1-GM26850.
2 Riverside Research Institute.

${ }^{3}$ Columbia University College of Physicians and Surgeons.

BB-6 The Analysis of A-Mode Ultrasound Waveforms with a OneDimensional Texture Algorithm, V. GONZALEZ, J. P. JONES, L. FERRARI, and M. BEHRENS, Department of Radiological Sciences, University of California-Irvine, Irvine, CA 92717.

We describe the results of an extensive analysis of $\mathrm{RF}$ and envelopedetected $A$-line ultrasound waveforms using a max-min measure for one-dimensional image texture as developed by Mitchell and his associates (O. R. Mitchell, C. R. Myers, and W. Boyne, IEEE Trans. on
Computers, C26, 1977). Our data base includes in vivo scans of the liver, pancreas, and spleen on over 30 human subjects and in vitro scans of yarious test objects and sponges. All measurements were accomplished with a computerized data analysis system designed for ultrasonic tissue characterization which we have implemented at UCI. Typically, we record 32 distinct $A$-lines which are 2048 points long (sampled at $20 \mathrm{MHz}$ ) through a specified region. A 1024 point window for each $A$-line is selected for processing. This data acquisition process is accomplished within the time required to produce a conventional $B$ scan image. The application of the Mitchell max-min measure to our data base demonstrates the ability of this algorithm to extract structural information about the object from the ultrasound echo signal. Such structural information concepts are confirmed by computer simulations of reflections from simple objects and the application of the Mitchell technique. The implications of such $A$-line signal structure analysis to ultrasonic tissue characterization appear to be significant and will be reviewed.

\section{PHOTOACOUSTIC IMAGING}

\section{Chairperson: R. L. MELCHER}

CC-1 Photoacoustic and Acoustic Imaging at High Frequencies, C. F. QUATE and C. WILLIAMS, Edward L. Ginzton Laboratory, Stanford University, Stanford, CA 94305.

A scanning photoacoustic imaging system has been implemented with an argon laser, intensity modulated at $940 \mathrm{MHz}$. We receive the radiation with an acoustic lens which is confocal with the optical beam. In photoacoustic imaging where the sound is generated by the absorbed light, we have imaged integrated circuits, stained biological cells and thin-film metal patterns. The resolution is approximately one micron. In a second mode, we have used this instrument for acoustic reflection imaging. We have learned that the reflection imaged can be dramatically altered when we heat with optical pulses coincident with the acoustic pulses. This technique also permits us to study the flow of the dc current through the object. The acoustic pulses are less than 20 nanoseconds in length. We are interested in such properties as adhesion to the substrate and changes in their properties during the process of laser annealing. We believe that these combined imaging modes may give us new insight into the physical behavior of thin films on transparent substrates.

CC-2 Ultrasonic-Wave Generation by Photoacoustic Absorption in a Thin Film between Two Media, G. C. WETSEL, JR., Department of Physics, Southern Methodist University, Dallas, TX 75275.

A one-dimensional theory of the generation of harmonic, traveling, ultrasonic waves by periodic heating of the interface between two media (backing and sample) has been extended. In the previous report, ${ }^{1}$ the heat source was assumed to be an infinitesimally-thick surface film with no material properties. For that model, the important backing and sample material-parameter groups for optimal signal enhancement were identified, and theoretical calculations were compared with reported experimental results. The extended model provides for a film of finite thickness and takes into account the film's appropriate optical, thermal, and acoustic parameters. The effects of these factors on ultrasonic-wave generation by photoacoustic absorption will be discussed for various combinations of backing, absorbing-film, and sample material parameters. Calculations of the ultrasonic-wave amplitude as a function of frequency for these combinations will be presented.

'G. C. Wetsel, Jr., 1980 IEEE Ultrasonics Symposium Proceedings, pp. 645-648, publ. by IEEE, New York, 1981 .

CC-3 Thermal Wave Diffraction Imaging, J. A. BURT, Department of Physics, York University, Downsview, Ontario, Canada, M 3J 1P3. 
Modulated heat waves produced in an opaque sample are employed to image a sample defect by detecting the heat wave diffraction pattern on the back surface of the sample. Theoretical calculations are presented for the near-field, highly attenuated diffraction pattern which, using holographic techniques, may be inverted to show the shape of the defect in the sample. In comparison with the photoacoustic microscope this technique allows deeper penetration of the sample by the heat waves, i.e., a lower modulation frequency, hence defects could be imaged from depths inaccessible to the photoacoustic microscope.

\section{CC-4 Thermal-Wave Microscopy of Semiconductors, A. ROSENC-} WAIG, Therma-Scan Co., Danville, CA 94526.

Thermal-wave microscopy is a new technique that produces microscopic images of surface and subsurface thermal features. Thermalwave microscopy provides unique and important capabilities for the semiconductor industry. These include the ability to detect subsurface defects, including interfacial flaws; the ability to perform nondestructive image depth-profiling; and the measurement of thin-film thicknesses with high accuracy. In addition to these capabilities, thermalwave microscopy allows the nondestructive detection and characterization of dopant regions in semiconductors, including, for the first time, the ability to obtain dopant depth profiles in a truly nondestructive fashion.

CC-5 Photothermal Spectroscopy on a Microscopic Scale, ${ }^{1} \mathrm{C}$. R. PETTS and H. K. WICKRAMASINGHE, Department of Electrical Engineering, University College, London, England.

We have adapted the technique referred to as photothermal or photoacoustic microscopy in the literature to record optical absorption spectra of biological objects point by point on a microscopic scale. The object to be imaged is attached to a thin Germanium sample which is electroded on both faces. The incident laser light is amplitude modulated-typically at $100 \mathrm{~Hz}$-and focused on to the sample. The periodic heating sets up a temperature wave within the Germanium which is picked up as a periodic change in resistance. The focused spot is scanned in a raster pattern in the usual manner and the detected signal modulates the brightness of a scan-synchronised CRO. The technique has proved to be extremely sensitive; by continuously tuning the laser wave length, it has been possible to record optical absorption spectra of biological objects on a microscopic scale.

' This work was supported by the Science Research Council.

CC-6 Experimental and Theoretical Characterization of near Surface Cracks in Solids by Photoacoustic Microscopy, ${ }^{1}$ P. K. KUO, L. J. INGLEHART, L. D. FAVRO, and R. L. THOMAS, Wayne State University, Detroit, MI 48202; M. SRINIVASAN, Carborundum, Niagara Falls, NY 14302.

A theoretical analysis is given for the photoacoustic signal from a solid containing a planar, lateral, subsurface crack. We calculate the magnitude and phase of the photoacoustic signal as a function of frequency. It is demonstrated that the phase of the signal can be used to provide a characteristic signature of such a crack as compared to other types of flaws such as vertical cracks or simple discontinuities in thermal properties. The theory is verified by analysis of data from fabricated planar flaws. This technique is applied to the photoacoustic microscopy of Knoop-indented SiC samples to detect the presence of subsurface lateral cracks. Both photoacoustic micrographs and computer-plotted perspective views of the Knoop signals are presented. We observe that the resolution of the Knoop cracks is much better than a thermal diffusion length. The physical reasons for this observation will be discussed.

${ }^{1}$ This research was sponsored by ARO under Contract DAAG29. 79-C-0151.
CC-7 Observation of a Sonoacoustic Effect using Piezoelectric Thermoacoustic Detection, H. I. RINGERMACHE ${ }^{1}$ and J. S. HEYMAN, NASA Langley Research Center, Hampton, VA 23665.

Thermoacoustic (TA) effects have been generated by optical, electromagnetic, and electron beam methods. In the present work, RF standing wave ultrasound is used in conjunction with FM techniques to generate low frequency thermoacoustics in a fused quartz cylindrical specimen. The TA signal amplitude is observed to vary as (modulation frequency) $)^{-1}$ and as (RF amplitude) $)^{2}$. This effect has possible application for thermal investigations in bulk, opaque materials.

${ }^{1}$ National Research Council Resident Research Associate.

CC-8 Radiation-Induced Thermoacoustic Soft Tissue Imaging, T. BOWEN, Department of Physics and Division of Radiation Oncology, University of Arizona, Tucson, AZ 85724.

It is known from the study of thermoelasticity by engineers, auditory sensing of microwave pulses by biomedical researchers, and acoustic detection of charged particles by physicists that the sudden thermal expansions due to heat pulses result in emission of acoustic waves. Recent progress in understanding the thermoacoustic detection of charged particles shows that a) there is a simple direct connection between the pattern of induced thermal stress and the received pressure signals, and that $b$ ) the signal-to-noise ratio of thermoacoustic signals relative to thermal noise can be reliably calculated. It is shown that these results are equally applicable for nonionizing radiations which deposit heat in short pulses, and indicate the feasibility of thermoacoustic imaging of soft tissue. If, as is expected, correlations exist between image features and tissue characteristics such as composition, structure and disease state, thermoacoustic imaging would permit a) the verification of treatment plans with respect to the positioning of therapeutic radiation dosage and $b$ ) noninvasive identification of tissue characteristics which ate not sensed by other imaging modalities.

\section{SURFACE AND BULK WAVE SENSORS}

\section{Chairperson: J. S. SCHOENWALD}

DD-2 Temperature Sensor using SAW Delay Line, ${ }^{1}$ D. HAUDEN, G. JAILLET, and R. COQUEREL, Laboratoire de Physique et Métrologie des Oscillateurs du C.N.R.S., associé à l'Université de Franche-ComtéBesançon, 25000 Besançon, France.

A new type of temperature sensor using SAW delay line is presented. Analysis of thermal properties of quartz-crystal enabled to find a doubly rotated cut with interesting properties (LST-cut). Delay lines obtained from LST-quartz crystal plates have a very linear frequencytemperature characteristic with a large sensitivity $\left(32 \mathrm{ppm} /{ }^{\circ} \mathrm{C}\right)$. Temperature sensor using such a delay line mounted in oscillating loop $(95 \mathrm{MHz})$ was built and its characteristic performances were measured. Measurements of response time show that it is twenty times faster than BAW quartz sensor (LC-cut) for the same frequency sensitivity $(0,1 \mathrm{~s}$ at 63,2 percent of frequency step). SAW delay lines enable to reach very high ultimate resolution $\left(50 \mu{ }^{\circ} \mathrm{C}\right)$ by increasing the frequency and by accounting the temperature on IF frequency af ter mixing with a reference oscillator. Following these properties, a new type of quartzthermometer is proposed.

${ }^{1}$ This work was sponsored by D.R.E.T. (France).

DD-3 Surface Acoustic Wave Acceloremeter, P. HARTEM ANN and P. L. MEUNIER, Thomson-CSF, Research Center, Domaine de Corbeville, 91401 Orsay, France.

This abstract deals with a particular surface acoustic wave (SAW) accelerometer structure loaded in flexure whose output signal is a fre- 
quency. An inertial mass, supported by flexible metal strips, is clamped between two thin circle-shaped quartz membrane operating in flexure. Dual $251 \mathrm{MHz}$ delay-line oscillators are laid on each membrane. In an acceleration field, the force, generated by the mass, induces local strain in their centers. So far the velocity of Rayleigh waves is changed and the mixed frequency output signal shifts proportionally to the force induced by the inertial mass. For $300 \mu \mathrm{m}$ thick $Y$ cut $10 \mathrm{~mm}$ diameter quartz plate, the sensitivity is close to $2 \mathrm{kHz} / \mathrm{G}$, the inertial mass being $13.8 \mathrm{~g}$. Mechanical resonance occurs at a frequency of $1.2 \mathrm{kHz}$. This accelerometer was tested over $\mathrm{a} \pm 20 \mathrm{G}$ range. Preliminary results about resolution threshold, linearity, and stability are reported.

DD-4 Pressure and Acceleration Sensitivity of a SAW Interferometer, R. E. DEWAMES, E. J. STAPLES, and J. WISE, Rockwell International, Thousand Oaks, CA 91360.

In this paper a new device configuration for sensing elastic deformation of a piezoelectric cyrstal using surface waves will be presented. Conventional surface wave sensors use either delay line or resonator structures and a change in phase or frequency is derived from the difference between expansion and second order elastic constants. By arranging grafting reflectors into a delay line configuration with large separations ( $>1000$ wavelengths), a SAW interferometer is formed. The passband response of such a device contains many closely spaced resonances similar to a comb filter response. Strain or accelerationinduced deformations in this case create amplitude as well as phase modulations in the surface waves. Theoretically, the device can be modeled using transmission line equivalent circuits. Experimental SAW interferometers were fabricated using widely separated one- and twoport SAW resonator structures at $175 \mathrm{MHz}$. Mode spacings were typically $1-1.5 \mathrm{kHz}$ apart. Deformation of the substrate produced large amplitude fluxuations and by counting "fringes" the crystal strain sensitivity was measured. Acceleration sensitivity under vibration was related to the phase and amplitude modulated sidebands produced. The results indicate this type of sensor may be of use where amplitude and not frequency variations as a function of strain are required.

DD-5 Sensors Utilizing Thin-Membrane SAW Oscillators, C. T. CHUANG and R. M. WHITE, Department of Electrical Engineering and Computer Sciences and Electronics Research Laboratory, University of California, Berkeley, CA 94720.

SAW oscillators employing propagation over membranes having thicknesses comparable to or smaller than a wavelength are of interest for application in integrated silicon sensors to measure quantities such as vapor density, pressure, etc. Since these membranes are thin, the wave energy is present at both the top and bottom surfaces, permitting the realization of enclosed devices where the waves can nevertheless interact with the ambient. Two regions of operation are considered: with the membrane thickness much smaller than a half-wavelength, a plate mode propagates and the wave energy is uniform along the membrane; with the membrane thickness comparable with or slightly larger than a half-wavelength, coupled modes propagate and the energy transfers periodically back and forth between top and bottom surfaces. Experimental results with a $1 \mathrm{MHz}$ SAW oscillator (wedge transducers on an aluminum plate having a thinned section) show that the loss on transmission onto a membrane as thin as 0.36 wavelengths is only $3 \mathrm{db}$, and the energies at the top and bottom surfaces are about equal for membrane thicknesses up to 0.8 wavelengths. The transmission and reflection at the start and end of the thinned section, and the effects of vapor condensation and surface loading are studied and related to the design of practical vapor sensors. Results on a $30 \mathrm{MHz}, \mathrm{ZnO}$-on-silicon thin-membrane SAW oscillator for sensor use are given.

DD-6 Circulating Flexural Wave Rotation Rate Sensor, ${ }^{1}$ H. F. TIERSTEN, D. S. STEVENS, and P. K. DAS, Rensselaer Poly technic Institute, Troy, NY 12181.
The natural frequency of flexural waves circulating in a ring resonator varies with the angular velocity of the ring on account of the Coriolis effect. If the direction of circulation is reversed the change in Iesonant frequency is reversed. Since the Coriolis effect is the only one that possesses this property, the influence of all other effects, even considerably larger ones, can readily be eliminated. Furthermore, since the change in frequency for a given angular velocity is inversely proportional to the frequency and the flexural waves are the lowest modes known; this should be the most favorable mode and configuration for an acoustic wave rotation rate sensor. Calculations are performed for flexural waves circulating in a fused silica ring using a very accurate perturbation procedure and the results are compared with measurement.

${ }^{1}$ Supported in part by NSF under Grants ENG 78-27637 and ECS79-18211.

DD-7 Graphic Sensor using a Lamb Wave, A. ISHII and S. HASHIMOTO, Musashino Electrical Communication Laboratory, N. T. T., Musashino-shi, Tokyo, 180 Japan.

A Lamb wave (plate wave) has been successfully used to establish an inexpensive and easy writing graphic input device. For this device, the $X$ and $Y$ coordinates of a pen tip drawing graphics on a paper laid on an input plate are measured by counting the time required for the Lamb wave to travel from the transducer to the pen tip. A fundamental mode, symmetrical Lamb wave was adopted because of its low phase velocity dispersion in the low frequency region. Low frequency operation leads to a small attenuation coefficient for the plate, and makes possible the use of low-cost plate materials such as acrylic resin. Thus, the Lamb wave device has advantages over SAW devices. The writing pen consists of a ball point pen tip, a piezoelectric ceramics transducer, and a brass tube connecting them. It can pick up the mechanical vibration of the plate, caused by arrival of the Lamb wave, when drawing graphics. A dual-threshold detection technique was devised to precisely and reproducibly detect wave fronts with low frequency spectrums. This graphic sensor is actually in use as the input device for a handwritten Chinese character recognition system in the laboratory.

\section{NDE SIGNAL PROCESSING}

\section{Chairperson: B. T. KHURI-YAKUB}

†EE-1 [Invited] Studies with Simulated and Real AE Signals, WOLFGANG SACHSE, Department of Theoretical and Applied Mechanics, Cornell University, Ithaca, NY 14853.

The constituents which comprise any acoustic emission (AE) system are the source, the structure or propagating medium, and the sensor with its associated electronics. This paper reviews the measurements which can be made with an ideal acoustic emission system that is comprised of a well-characterized step source, a structure for which the Green's function has been computed, and a broadband ultrasonic displacement sensor. Such a system is over determined, permitting each of the components to be changed, and its characteristics determined by signal deconvolution techniques. In this way, the emission source function, the dynamic Green's function of the structure, and receiving transducer's transfer function can be determined.

Results are presented of actual force-time functions for various simulated and actual emission sources operating in a plate. The sources characterized include the fracture of brittle materials and the impact of spheres and electrical arcs on the surface of a plate and the opening of a crack. Results are also presented of experimentally determined transfer functions of various $\mathrm{AE}$ sensors, as well as the dynamic Green's functions of thick flat plates for various source and receiver locations on its surface. 
EE-2 Counteracting Multipath Effects in Gap Measurement of Nuclear Steam Generator Tubes, ${ }^{1}$ I. AMIR, V. L. NEWHOUSE, and E. S. FURGASON, Purdue University, West Lafayette, IN 47907.

A technique to measure the growth of support plate corrosion around the steam generator tubes of nuclear reactors is developed. An analysis of the backscattered echoes from a finite number of thin layers produced by an ultrasonic pulse-echo system is used to analyze the problem. It is shown that multipath effects and corrosion cause the severe reduction in signal-to-clutter ratio which, in the past, prevented isolation of corrosion echoes. A new transducer assembly using an ellipsoidal mirror is discussed. This type of assembly overcomes problems of multipath effects and inhomogeneity of the support plate. Using the described transducer assembly, several corroded samples were tested. The results, after applying a signal-to-noise ratio enhancement technique, provide an accurate profile of the samples.

${ }^{1}$ This work was supported by EPRI-Steam Genarator Project Office.

EE-3 High-Speed Digital Golay Code Flaw Detection System, B. B. LEE and E. S. FURGASON, Electrical Engineering Department, Purdue University, West Lafayette, IN 47907.

Current ultrasonic correlation flaw detection systems are limited to slow operation speed because long correlation times are required to reduce self-noise to tolerable levels. Self-noise occurs due to incomplete pulse-compression in the correlation detector and causes interference when a flow is located next to a large surface or in a very grainy material. A new, entirely digital, flaw detection system is described which uses paired pseudorandom codes called complementary Golay codes, as the transmit signals, to cancel self-noise in two transmit bursts. Results are presented which show that the Golay code system produces better performance under the presence of grains and large surfaces, and operates at much higher speed, than previous correlation flaw detection systems. The new Golay code system is also shown to have the same resolution as conventional pulse-echo systems and to obtain the same high signal-to-noise ratio enhancement as previously described random signal correlation flaw detection systems.

EE-4 Flaw Visibility Enhancement by Split-Spectrum Processing Techniques, ${ }^{1}$ N. M. BILGUTAY, V. L. NEWHOUSE, and E. S. FURGASON, Purdue University, West Lafayette, IN 47907.

When the range cell of an ultrasound system contains many unresolved and random reflectors such as grains, the overlapping echoes which result make the detection of flaws within the range cell difficult even when the flaws are substantially larger than the grains. Since the grains are small and closely spaced, their echoes result in a complicated interference pattern which is significantly more sensitive to shifts in the transmitted frequency compared to flaws which are much larger in size. Therefore, decorrelation in grain echoes can be obtained by shifting the transmitted frequency. This basic principle has led to the development of a split-spectrum processing technique for an ultrasonic flaw detection system which improves the flaw-to-grain echo ratio in large grained materials. The enhancement is achieved by partitioning a wideband received spectrum to obtain frequency shifted bands, which are then processed to suppress the grain echoes with respect to the flaw echo using a novel minimization and conventional averaging algorithms. Experimental data for titanium and stainless steel samples have been obtained showing improved flaw detection capabilities.

'Supported by Purdue NSF-MRL Program and EPRI.

EE-5 Analysis Methods for Ultrasonic Inspection of Steam Turbine Discs, T. E. MICHAELS and J. E. MICHAELS, JTM Systems and Consulting, Inc., lthaca, NY 14850, and G. E. RONCA, Westinghouse Electric Corporation, Philadelphia, PA 19113.
Reliable ultrasonic inspection of steam turbine discs is an important objective in the power generation industry. This inspection task is difficult because the complicated disc geometry gives rise to many false indications, the exact ultrasonic beam path is difficult to determine due to thick sections and high internal stresses, and physical access to the discs is quite restricted. Analysis methods and inspection equipment for improving defect detection and characterization have been developed as part of an ongoing Westinghouse program for developing an automated field system. Analysis methods being evaluated include feature analysis, waveform reconstruction (averaging), pattern recognition, and deconvolution. Equipment items include a multiprocessor computer system, a 5-axis mechanical scanner, ultrasonic instrumentation, control electronics, and a real-time ultrasonic imaging system. This paper presents ultrasonic inspection data and analysis results, and briefly describes the equipment.

EE-6 Improvement of the Signal-to-Noise Ratio, Flaw Classification and Reconstruction with Data from Automatic Ultrasonic InspectionALOK, ${ }^{1}$ O. A. BARBIAN, B. GROHS, W. KAPPES, H. PAUL, and R. LICHT, Fraunhofer-Institut für zerstörungsfreie Prüfverfahren, Universität, Geb. 37, D-6600 Saarbrücken 11, FRG.

The ALOK system (Amplituden-Laufzeit-Ortskurven, amplitude and transit time locus curves) has a new concept to obtain and to evaluate data from automatic ultrasonic inspection of thick-walled components, for instance reactor pressure vessels. This system has two essential parts: 1) Noise elimination of the measurement values and therewith improvement of the signal-to-noise ratio by formation of transit time locus curves and 2) reconstruction of flaw position and dimension only from the noise-eliminated transit time measurement values. Within this system you can use each multiple-probe system, i.e., probe systems with the pulse-echo technique with beam incidences of, e.g., $0^{\circ}, 45^{\circ}$, $60^{\circ}, 70^{\circ}$ using single crystal or TR-probes with wide-spread beams as well for longitudinal as for shear waves, and the V-through-transmission technique and the tandem technique; and one or several phases array probes.

This paper will give a short overview of the principle of the ALOK system and explain this by experiments and inspections of components with artificial and natural flaws.

1 This work is supported by the Federal Ministry of Research and Technology of FRG.

\section{MEDICAL ULTRASOUND: TRANSDUCERS AND IMAGING}

\section{Chairperson: J. G. MILLER}

FF-1 [Invited] Hydrophones for Ultrasonic Dosimetry, ${ }^{1}$ R. A. BANJAVIC and P. L. CARSON, University of Colorado Health Sciences Center, Department of Radiology, School of Medicine, Denver, CO 80262 .

The need for precise and reproducible ultrasonic dosimetry measurements is necessitated by groups involved both in acoustic bioeffects research and the manufacturers and users of clinical diagnostic ultrasound equipment. For work in each area, knowledge of the point pressure temporal waveforms at various spatial locations in the field of a transducer becomes paramount. These distributions when squared and integrated provide the basis for the determinations of the temporal and spatial intensities in the field of interest. Miniature piezoelectric receiving hydrophones now exist which can perform these measurements within reasonable uncertainty limits provided certain information about their behavior is known. This information includes the pressure and intensity response of the hydrophone as a function of frequency, the linearity of the pressure response as a function of intensity and the temporal and thermal stability of these responses. The results of a vigorous 
evaluation of various hydrophones to provide this information consistently will be discussed. The relationship between active element construction (ceramic PZT versus polymer PVDF) and the overall performance of the hydrophone to reproduce faithfully the pulsed pressure waveforms in time and space also will be presented.

${ }^{1}$ This work was supported in part through a contract with NEMA.

FF-2 Computer-Optimized Design of Quarter-Wave Acoustic Matching and Electrical Matching Networks for Acoustic Transducers, ${ }^{1}$ A. R. SELFRIDGE, R. BAER, B. T. KHURI-YAKUB, and G. S. KINO, Ginzton Laboratory, Stanford University, Stanford, CA 94305.

We have developed an iterative computer program which can optimize the parameters for the acoustic matching layer(s) and the electrical matching network of a transducer for a minimum length of the pulse response. By including the interactions between electrical and acoustic matching in our model, we have been able to improve the quality of our transducers significantly. A $3.5 \mathrm{MHz}$ center frequency transducer was constructed to verify the computer results. The transducer was made of PZT-5A and had a low impedance epoxy backing. The designs of a single quarter-wave matching layer and a three-element tuning network were optimized on the computer to obtain both a short ringdown time and a small insertion loss. This transducer's characteristics closely matched those predicted by the theory. The impulse response was approximately $2 \frac{1}{2}$ cycles long with a round trip insertion loss of $7 \mathrm{~dB}$ at the center frequency; and the $40 \mathrm{~dB}$ ringdown time was $1.5 \mu \mathrm{s}$.

${ }^{1}$ This work was supported by the Air Force Office of Scientific Research under Contract F49620-79-C-0217 and the Department of Energy under Contract DE-AM03-76SF00326.

FF-3 A New Method for Estimating the System-Independent Characteristics of an Ultrasonic Piezoelectric Transducer, TADASHI KOJIMA, Nihon Dempa Kogyo Co., Ltd., Sayama-shi Saitama-ken 350-13, Japan.

As the Ultrasonic piezoelectric transducer has been widely used for medical diagnosis as well as nondestructive inspection, it has been desired to establish a reasonable method which can estimate the proper characteristics of the transducer itself. For estimation of the systemindependent characteristics of an ultrasonic piezoelectric transducer in a pulse-echo system, voltage transfer gain (VTG) is introduced, which is related to the transfer function between the input voltage of the transmitting transducer and the open circuit output voltage of the receiving transducer. The VTG characteristics represent the characteristics of a transducer itself, and self-reciprocity calibration of the transducer can be calculated with the VTG, the reciprocal parameter and the input impedance of the transducer. A design example of $2.5 \mathrm{MHz}$ transducer is discussed through the VTG estimation method, and it is shown that experimental results agree well with the theoretical calculations.

FF-4 Lateral Resolution Improvement of Focused Single Piston Transducers by a Signal Processing Technique, ${ }^{1}$ G. W. KEILMAN and H. B. BUTTS, Advanced Technology Laboratories, Inc., P.O. Box 6639 , Bellevue, WA 98007.

Traditional focused single piston transducers used in pulse-echo systems are limited in lateral resolution by diffraction. If the lateral beam profile is known and adjacent overlapping azimuthal echo samples are available, it is possible to process these adjacent samples to reduce the lateral beamwidth. This paper will explore some of the limitations of these techniques in a tissue-equivalent at tenuating medium. The effect of the processing on the beam in the elevation direction is discussed. Preliminary experimental results are presented. Inc.
FF-5 A Recursive Scheme for Improvements of the Lateral Resolution in B-Scan Ultrasonography, SUN I. KIM, BYOUNG G. MIN, and JANG H. YOUN, Department of Biomedical Engineering, Seoul National University Hospital, Seoul, Korea, and MYOUNG S. KO, Department of Inst. and Cont. Seoul National University, Seoul, Korea.

The objective of this paper is to present a digital method for improving the lateral resolution of the B-scan images in the medical applications of ultrasound. The method is based upon a mathematical model of the lateral blurring caused by the finite beamwidth of the transducers. This model provides a simple method of applying a recursive scheme for image restoration with fast computation time. The point spread function (PSF) can be measured by the reflective signals after scanning the small pins located along the depth of interest. From the measured PSF, one can compute the coefficient matrices of the inverse discrete-time dynamic state variable equation of the blurring process. Then, a recursive scheme for deblurring is applied to the recorded Bscan image to improve the lateral resolution. One major advantage of the present recursive scheme over the transform method is in its applicability for the space-variant imaging, such as in case of the rotational movement of transducer.

FF-6 Calibration and Performance Evaluation of Miniature Ultrasonic Hydrophones using Time Delay Spectrometry, PETER A. LEWIN, Technical University of Denmark, Bldg. 404, DK-2800 Lyngby, Denmark.

In the past few years, miniature ultrasonic probes have gained attention and recognition as convenient tools in the field of ultrasonic dosimetry. One of the key parameters-free field voltage sensitivity of the probe expressed as a function of frequency-is usually determined at discrete frequencies, of ten separated by more than one megahertz in the frequency range considered (typically $1-10 \mathrm{MHz}$ ). This discrete frequency calibration technique suffers from the fact that it frequently overlooks rapid and significant variations in the frequency response of the probe due to, e.g., spurious mechanical resonances or radial mode resonances. This paper describes a novel calibration procedure which overcomes the above mentioned shortcoming and allows determination of the continuous frequency response of the miniature ultrasonic hydrophone. The procedure utilizes time delay spectrometry (TDS) technique. The principles of the TDS technique will briefly be outlined, and the results of the calibrations performed including frequency response curves and directivity patterns of the ultrasonic probes will be presented. Advantages of the TDS technique will be pointed out, and the basic limitations of the technique will be discussed.

\section{SAW FILTERS I}

\section{Chairperson: W. R. SHREVE}

†GG-1 Saw Filter Requirements for Clock Recovery in Digital LongHaul Optical Fiber Communication Systems, R. L. ROSEN BERG, D. G. ROSS, and P. R. TRISCHITTA, Bell Laboratories, Holmdel, NJ 07733.

A long-haul optical transmission system utilizes cascaded single-mode fiber spans tens of kilometers in length. Each span is followed by an electronic regenerator that receives, reconstructs, and retransmits the original message after it has been attenuated, time-dispersed, and otherwise degraded in the fiber span. In the widely used digital signaling format, the message reconstruction process requires a clock running at the signaling rate. The clock waveform is presently recovered from the pulse train itself, typically with the aid of a SAW filter. We report a study of the SAW filter requirements in ocean and terrestrial long-haul systems as determined by system-terminal characteristics, operating 
temperature range, clock recovery circuitry, timing jitter effects, and handling stresses. Results obtained at $274 \times 10^{6} \mathrm{bit} / \mathrm{s}$ with a coupledresonator SAW clock-recovery filter are presented. Filter-related items needing further wotk are summarized.

GG-2 The Diffraction Analysis and Correction of Narrowband Saw Transversal Filters, R. C. PEACH, N. H. DOGGETT, F. S. McCLEMONT, and A. KATSELLIS, The General Electric Co. Ltd, Hirst Research Centre, Wembley, Middlesex, England.

It is common practice in the design of narrowband SAW transversal filters on quartz to incorporate weighting on both transducers. This technique can make a reasonably rigorous diffraction analysis of the overall structure prohibitively expensive in computer time. A method has been developed to overcome this problem and to allow a complete diffraction analysis even when both transducers are of comparable length. This method has been applied to a $200 \mathrm{MHz}$ filter on quartz with a 0.3 percent fractional bandwidth, and it has been found necessary to introduce extensions to the basic free diffraction model to obtain agreement with experiment. Diffraction amplitude and phase correction has been employed in addition to careful suppression of unwanted acoustic reflections within the device. With these precautions it has been possible to obtain a clean response with stopband levels of $70 \mathrm{~dB}$.

GG-3 Implementation of Reflective Array Matched Filters for Radar Applications, J. L. THOSS, D. PENUNURI, and M. THOSTENSON, Hughes Aircraft Co., Fullerton, CA 92634.

Utilizing weighted reflective dot arrays, two types of narrow-band matched radar filters are realized on $S T$-quartz. 1) A bandpass filter matched to a $10-\mu$ s transmitted pulse, having a 0.25 percent operating bandwidth centered at $30 \mathrm{MHz}$, demonstrates outstanding phase linearity, better than 0.5 degree rms phase error, and extremely low mismatch loss, less than $0.1 \mathrm{~dB}$, an improvement of approximately $0.5 \mathrm{~dB}$ over other filter designs. 2) A dispersive matched filter producing a 100- $\mu$ s long nonlinear FM waveform, having 3 percent operating bandwidth centered at $32 \mathrm{MHz}$, exhibits excellent time sidelobe suppression exceeding $30 \mathrm{~dB}$. This filter incorporates a novel low-cost frequency trimming technique employing thin dielectric films to fine adjust the phase velocity. The results from a set of experiments determining 1) the effective phase velocity as a function of reflective array metal (Au) thickness and 2) the single stripe reflection coefficient constant $\left(r=c h / \lambda_{0}\right)$ for gold films are discussed.

GG-4 Active Matching of Monolithic ZnO on Si Interdigital Trans ducers, ${ }^{1}$ A. F. ARBEL ${ }^{2}$ and J. E. BOWERS, Ginzton Laboratory, Stanford University, Stanford, CA 94305.

Active matching circuits have been developed for matching SAW IDT's to $50-\Omega$ generators and receivers. For the output circuit, the use of a low input impedance receiver allows the elimination of the inductor usually needed to tune out the IDT capacity. The frequency dependence of the radiation impedance is compensated by a single stage active circuit. Similarly, a low output impedance driver with frequency dependent gain is used to directly drive the IDT. A 3-dB bandwidth of $50 \mathrm{MHz}$ has been achieved with $\mathrm{ZnO}$ on Si Sezawa wave IDT's operating at a center frequency of $155 \mathrm{MHz}$. The linear dynamic range is $60 \mathrm{~dB}$. The use of low impedance drivers and receivers reduces the magnitude of the reflected surface wave from each IDT. For convolvers and storage correlators, this results in a smaller self-convolution signal, while for delay lines this results in a larger triple transit loss. These matching techniques are applicable to IDT's on other materials and also to bulk acoustic wave transducers.

1 This work was supported by the Office of Naval Research (NO0014 76-C-0129).
${ }^{2}$ On leave from Israel Institute of Technology, Department of Electrical Engineering, Haifa Technion City, 32000, Israel.

GG-5 A 4.4 GHz SAW Filter Using a Single-Crystal ZnO Film on Sapphire, T. MITSUYU, O. YAMAZAKI, and K. WASA, Materials Research Laboratory, Matsushita Electric Industrial Co., Ltd., Moriguchi 570, Japan.

A 4.4 GHz SAW filter has been fabricated using a single-crystal film of $\mathrm{ZnO}$ on sapphire substrate. The $\mathrm{ZnO}$ film was successfully grown by an if-magnetron-sputtering technique. SAW properties of the $\mathrm{ZnO} /$ sapphire structure have been found to well agree with theoretical predictions. Interdigital transducers with $0.5 \mu \mathrm{m}$-width double electrodes were used to construct the filter. An application of the first higherorder mode (Sezawa-mode) with extremely high phase velocity and third-order harmonic generation by the double electrodes provide such high-frequency operation in spite of relatively wide electrodes. The filter shows a $3 \mathrm{~dB}$ band-width of $50 \mathrm{MHz}$ and an insertion loss of 16 $\mathrm{dB}$ at matched condition. The temperature coefficient of delay has been measured to be $35 \mathrm{ppm} /{ }^{\circ} \mathrm{C}$ for the present filter. The fabrication process and fundamental SAW properties of the $\mathrm{ZnO} /$ sapphire structure, as well as the characteristic of the filter, will be presented.

GG6 Surface Wave Pulse Compression Filters with Long Chirp Time, H. R. STOCKER, R. VEITH, and E. WILLIBALD, Siemens AG, Research Laboratories, Otto-Hahn-Ring 6, D-8000 München 83, and G. RIHA, Institut für Allgemeine Elektrotechnik und Elektronik, TU Wien, GuBhausstr. 27-29, A-1040 Wien.

Pulse compression filters for radar have chirp durations up to $50 \mu \mathrm{s}$ and time/bandwidth-products $\leqq 100$. Typically sidelobe suppressions in the compressed pulse are $-30 \mathrm{~dB}$ for interdigital transducer filters. Higher suppressions of $-40 \mathrm{~dB}$ were achieved with reflective array compressors but only with a phase compensation in an additional production step. We have developed 17- $\mu$ s surface wave chirp filters for a pulse compression system using a double expansion technique. The projected sidelobe suppression was $-37 \mathrm{~dB}$. In order to fulfill the requirements on the phase accuracy a new production technique was used. The transducers were designed with identical groups of equally spaced electrodes. Thus, needing only one reticle a $10: 1$ wafer stepper quickly printed the complete transducers group by group on a $S T$-cut quartz substrate. In some cases remaining phase errors in the impulse response of the filters could be correlated with crystal defects shown by $\mathrm{X}$-ray topography. Investigations of different kinds of substrates showed that only $S T$-cuts from so called $x$-bars did not degrade the phase response.

\section{ACOUSTOELECTRIC DEVICES}

\section{Chairperson: R. W. RALSTON}

HH-1 Recent Developments in the ZnO on Si Storage Correlator, ${ }^{1}$ R. L. THORNTON, J. E. BOWERS, and J. B. GREEN, Ginzton Laboratory, Stanford University, Stanford, CA 94305.

In this paper we describe various improvements in the basic structure of the monolithic storage correlator. At the present time the three major areas of improvement open to these devices are device bandwidth, storage time, and spurious signal level. We have investigated all of these avenues of improvement. In order to take advantage of the increased potential bandwidth of Schottky diodes, we have fabricated Sezawa wave correlators. This presents the possibility, with proper tuning, of obtaining 20-30 percent bandwidths. We have succeeded in raising the barrier height of PtSi on Si Schottky diodes from 0.86 volt up to 1.0 volt by ion implantation. This offers the possibility of retaining the $1 \mathrm{~ns}$ read-in times of Schottky diodes while increasing storage times by 
two orders of magnitude. We have also developed a technique for suppressing the spurious acoustic signals generated by the top-plate during the read-out process. This technique relies on the high conductivity of the PtSi metallization of the diodes to allow the top-plate to be placed outside of the acoustic beam path and not on the $\mathrm{ZnO}$, thus eliminating any spurious signals generated by the edges of the top plate.

\section{${ }^{1}$ This work was supported by the Office of Naval Research under Contract N00014-76-C-0129.}

$\dagger$ HH-2 The SAW/FET: A New Monolithic Buffer Memory, ${ }^{1}$ J. B. GREEN and G. S. KINO, Ginzton Laboratory, Stanford University, Stanford, CA 94305 and J. T. WALKER and J. D. SHOTT, Integrated Circuit Laboratory, Stanford University, Stanford, CA 94305.

We report on the fabrication of a new monolithic buffer memory using the zinc oxide on silicon surface acoustic wave technology. The device is based on an interleaved NMOSFET array multiplexed by two independent single-bit shift registers. The 700 individual FET's have large area sources $(1 \mathrm{~mm} \times 5 \mu)$ which, by virtue of their junction capacitance, act as storage nodes for an analog waveform. A layer of $c$ axis oriented zinc oxide is sputter deposited on top of this FET array. A metal film defines the top-plate electrode and interdigital transducers as in an ordinary monolithic storage correlator. High-frequency (100 $\mathrm{MHz}$ ) signals input into one of the interdigital transducers can be stored in the FET array. These waveforms can then be read out of the device at a comparatively slow data rate (around $100 \mathrm{KHz}$ ) by clocking the shift registers and observing the common drain connection of the FET's. Thus we have the capability of processing a high speed waveform at slow computer-compatible data rates. Experimental results will be reported.

1 This work was supported by the Office of Naval Research under Contract No. N00014-75-C-0632.

HH-3 Bias Stable ZnO-on-Si SAW Devices, ${ }^{1}$ R. D. CHERNE, M. R. MELLOCH, R. L. GUNSHOR and R. F. PIERRET, School of Electrical Engineering, Purdue University, West Lafayette, IN 47907.

Charge injection and trapping within the metal- $\mathrm{ZnO}-\mathrm{SiO}_{2}-\mathrm{Si}$ (MZOS) structure is the well-known cause of a serious bias instability routinely exhibited by $\mathrm{ZnO}-\mathrm{on}-\mathrm{Si}$ surface acoustic wave devices. Herein we report and describe the attainment of dc stable MZOS device structures. Through a simple modification in the standard MZOS fabrication procedure devices have been produced which, under dark conditions, attain a repeatable dc operating point within seconds after applying the desired dc bias. Fabrication details, data illustrating the achieved degree of bias stability, and comparative SAW device characteristics are presented. A physical model explaining the observed stability is also discussed.

${ }^{1}$ This work was supported by the National Science Foundation under Grant ECS-8103744 and the Air Force Office of Scientific Research under Grant A FOSR-77-3304.

HH-4 Experimental Observation of Charge Transfer by Surface Acoustic Waves in a Monolithic Metal/ $\mathrm{ZnO} / \mathrm{SiO}_{2} / \mathrm{Si}$ (MZOS) Device, ${ }^{\prime} \mathrm{F}$. L. AUGUSTINE, R. J. SCHWARTZ, and R. L. GUNSHOR, Purdue University School of Electrical Engineering, West Lafayette, IN 47907.

Observation of minority carrier transport in the MZOS device is reported. Less than ideal device performance such as excessive time delay, charge spreading, and low charge capacity are explained in terms of high sensitivity to surface state traps. Operation of the device has been achieved by optical generation of minority carriers as well as by injection by means of a p- $n$ junction. Lateral bias effects and charge loading of the SAW are also described.

${ }^{1}$ This work was supported by an NSF-MRL Grant DMR 77-23798.

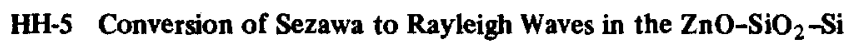
Configuration, ${ }^{1}$ M. R. MELLOCH, R. L. GUNSHOR, and R. F. PIERRET, School of Electrical Engineering, Purdue University, West Lafayette, IN 47907.

The high electromechanical coupling of the Sezawa mode makes it feasible to construct devices such as a multistrip coupler in the metal$\mathrm{ZnO}-\mathrm{SiO}_{2}-\mathrm{Si}$ surface acoustic wave configuration. The transmission of a 21.8- $\mu \mathrm{m}$ wavelength Sezawa surface wave through an 8.1- $\mu \mathrm{m}$ aluminum grating multistrip coupler, however, exhibited a narrow stopband. This stopband has been identified as resulting from a conversion of the Sezawa mode to the Rayleigh mode. Clearly, the mode conversion must be taken into account in the design of periodic grating structures, such as a multistrip coupler, to avoid spurious stopbands. The conversion also provides a new phenomenological basis for the construction of narrow-band filters.

1 This work was supported by the Air Force Office of Scientific Research under Grant AFOSR-77-3304, the National Science Foundation under Grant ECS-8103744, and the NSF-MRL Grant DMR 77-23798.

HH-6 Surface Acoustic Wave Parametric Oscillation in Si-on-LiNbO YASUHIKO NAKAGAWA, Faculty of Engineering, Yamanashi University, Kofu, 400, Japan.

Theoretical and experimental results are reported for a parametric oscillation of surface acoustic wave (SAW) in Si-on- $\mathrm{LiNbO}_{3}$. This structure has large efficiency of the SAW convolver which has a reverse process of the nonlinear effect in the SAW parametric oscillation. Therefore, the generation of the SAW $(\omega, \pm k)$ with a spatially uniform electric field $(2 \omega, 0)$ applied to the $\mathrm{Si}-\mathrm{on}-\mathrm{LiNbO}_{3}$ substrate has been easily successful. The threshold power required for parametric oscillation can be substantially reduced by the highly efficient SAW reflectors. The coupled mode equations describing the SAW's are solved using the appropriate boundary conditions, and we get the start oscillation threshold power and the growth rate in time numerically. Experimental frequency $\omega$ was $130 \mathrm{MHz}$, and the conversion efficiency from the pumping to the SAW was about $-23 \mathrm{~dB}$ at the threshold power of 14.4 $\mathrm{dBm}$. It is possible to apply this nonlinear effect to a switching device, spectrum clipper, and frequency-selective limiter, etc.

\section{MEDICAL ULTRASOUND: TRANSDUCER ARRAYS}

\section{Chairperson: R. H. TANCRELL}

Il-1 [Invited] Nonideal Radiators in Phased Array Transducers, J. D. LARSON, Hewlett-Packard Company, Palo Alto, CA 94304.

One dimensional arrays for medical acoustic imaging are most simply understood from the point of view of a group of point radiators. The individual radiators (transducer elements) are modeled as single mode, piston vibrators with negligible cross-coupling to their neighbors. It is relatively straightforward to calculate the acoustic beam response-beam steering, focussing, and resolution-from such a model. When one builds real world arrays with proper element density to suppress the grating lobes, the situation becomes much more complex. Cross-coupling between elements and multimoding of the individual elements become the dominant problems. The beam response then departs from the ideal. This talk will discuss some of the sources of cross-coupling, both electrical and acoustical, and explore suppression techniques. The question of vibration modes in a single element will be explored, in particular the relation between the nonideal behavior of the array elements and the resulting beam response deficiencies.

II-2 Properties for a 2D Multiplexed Array for Acoustic Imaging, ${ }^{1} \mathrm{~J}$. F. GELLY and C. MAERFELD, Thomson-CSF DASM Cagnes/Mer, 06802 France. 
A new technique of making a $2 \mathrm{D}$ array with a built-in switching circuit has been proposed in a previous paper presented at the 1980 Ultrasonic Symposium. A 2-MHz $16 \times 16$ array has been realized on these principles, and its main characteristics and limitations are presented here. The array will be used in the focal plane of an acoustic lens. A good uniformity between each elementary cell is achieved: all sensitivities are within $\pm 1 \mathrm{~dB}$, and the $3 \mathrm{~dB}$ directivity angle is constant around $25^{\circ}$. Crosstalk mechanisms are analyzed: on one hand between adjacent transducers, crosstalks are induced by the acoustic radiation and capacitive coupling, on the other hand, between elements far apart, crosstalks are given by the multiplexing circuits. The $\lambda / 4$ layer drastically modifies these properties. Transient signals which appear during switching limit either switching rate or detectivity. Finally, this type of $2 \mathrm{D}$ retina appears to be easily feasible and well suited to the application. It will be extended to a $64 \times 64$ array in the near future.

${ }^{1}$ This work has been sponsored by DRET.

II-3 The Combination of the Emission and the Reception Directivity of Pulsed Linear Sonographic Arrays, P. J. 'T HOEN, Philips, Medical Systems Division, Best, The Netherlands.

In linear-array imaging for medical purposes, pulsed emission is used. The two-way directivity is then not simply the product of the emission and the reception directivity. This would be the case with $\mathrm{CW}$ excitation, as then the same frequency is received. But with pulsed emission the frequency content of the travelling pulse depends on the position in space, as the directivity is frequency dependent. One can loosely state that "space acts as a spatially variant filter." According to the results of calculations for a range of emission and reception geometries, the two-way directivity is generally worse than the product of its constituents.

II-4 The Design of an Auto-Dynamic-Focusing Annular Array, R. Y. LIU, W. V. HARRISON and M. H. WILCOX, ADR Ultrasound, Tempe, AZ 85282 .

A dual-element spherical annular array, which is formed by cutting a single spherical disk transducer into a center disk and an annular piece, has been designed to achieve two-zone dynamic focusing automatically without using any phasing or timing delay electronics. The design is based upon the principle that spherical disk transducers having the same radius of curvature, but different diameters, have different acoustic focal points. By reducing the aperture size, the focal zone is shifted toward the transducer. Therefore, during the receiving process of the pulse-echo imaging operation, two-zone focusing is achieved by activating the center disk only for the near field zone, and activating both elements simultaneously for the far-field zone. Such auto-dynamicfocusing has also been applied to a multielement multifocal-zone spherical annular array. It not only greatly simplifies the design approach, but also eliminates quantization errors inherent in electronic dynamic focusing systems. The design considerations are based on 3D simulated wideband pulse-echo beam profiles, and the experimental results obtained from a computer-controlled ultrasound beam profile measurement system. The applications of this array in the field of medical ultrasound are also presented.

II-5 Resolution of Fresnel Phase Plate Transducers, B. NOORBEHESHT, Department of Electrical Engineering, University of Houston, Houston, TX, and M. MORTEZAIE and G. WADE, Department of Electrical and Computer Engineering, University of California, Santa Barbara, CA.

Ultrasonic imaging transducers have been proposed in which a piezoelectric plate is used in conjunction with a Fresnel phase plate (FPP) to produce a focused beam of ultrasound. Focusing is achieved with these devices by polarizing and electroding the piezoelectric plate in such a way as to produce a near-field pattern in the shape of an FPP. The focal plane distribution of these transducers cannot, in general, be predicted by diffraction theory alone. This is because the acoustic field pattern in front of the transducer is not an exact FPP pattern. We have previously shown that the above effect, called mechanical aberration, causes the resolution of Fresnel zone plate (FZP) transducers to be poorer than their predicted diffraction-limited resolution. In this paper we show that mechanical aberrations have a similar effect on the resolution of FPP transducers. However, in the present case both magnitude and phase characteristics of the transducer's aberration-limited transfer function are given. For the FZP transducers only the magnitude was provided. The effects on resolution of material properties and other device parameters are discussed. Experimental results are also presented.

\section{SAW FILTERS II}

\section{Chairperson: H. van de VAART}

JJ-1 Wideband Third Harmonic Chirp Filters, R. B. STOKES, K. F. LAU, K. H. YEN, and R. S. KAGIWADA, TRW Electronics and Defense, Redonodo Beach, CA 90278.

Wideband microscan receivers with high probability of intercept must include either many separate processing channels or very wideband chirp filters. The small linewidths necessary for SAW filter bandwidths of $500-1000 \mathrm{MHz}$ usually lead to expensive processing techniques, such as electron beam lithography. Inexpensive third harmonic chirp transducers can be fabricated by ordinary photolithography with bandwidths above $1 \mathrm{GHz}$. Chirp transducers provide three times the time-bandwidth product at third harmonic as at fundamental. A third harmonic slanted transducer chirp filter with $500 \mathrm{MHz}$ bandwidth and 0.25 microsecond dispersive delay is reported here. Performance of this device and design techniques are discussed.

$\dagger$ JJ-2 The Deep Inlaid Interdigital Transducer, R. CHAPMAN, Plessey Company, Allen Clarke Research Laboratories, Caswell, Towcester U.K.; E.G.S. PAIGE and M. W. WAGG, Department of Engineering Science, Oxford University, Oxford, U.K.

While surface skimming bulk wave devices exhibit many of the attributes of bulk wave devices, acoustoelectric conversion efficiency has the low values associated with surface waves rather than bulk waves. This is a consequence of the use of the conventional interdigital transducer (IDT). In this paper a new IDT structure is described having fingers inlaid to depths comparable to a wavelength. Compared to a conventional IDT this deep inlaid transducer offers increased fractional bandwidth at minimum insertion loss together with diminished sensitivity to stray capacity. Preliminary experimental results are presented for inlays up to $\lambda / 4$ deep on $-51^{\circ}$ rotated $Y$-cut quartz.

\section{JJ-3 Experimental and Theoretical Analysis of Temperature Depen-} dence of Wideband SAW RAC Devices on Quartz, ${ }^{1}$ D. M. BOROSON and D. E. OATES, Lincoln Laboratory, Massachusetts Institute of Technology, Lexington, MA 02173.

We present data and analysis of the temperature dependence of wideband SAW RAC devices fabricated on $S T$ quartz and RAC-cut quartz. Both cuts are of interest for temperature-stable RAC's because $S T$ is temperature compensated in the long direction and RAC cut is compensated in two directions. The theoretical RAC response has been extended to include temperature dependent anisotropies in order to model $S T$ quartz. The new madel predicts that, to first order, the chirp slope is insensitive to changes of delay in the short, uncompensated direction. Thus the chirp slope of a RAC on $S T$ quartz is first- 
order temperature compensated. There is, however, an increase in device attenuation due to the anisotropy. The analysis of RAC-cut quartz is identical to that of an isotropic material because of crystal symmetry. Measurements of chirp slope, group delay and insertion loss have been made on an $S T$ quartz RAC, $f_{0}=100 \mathrm{MHz}, \Delta f=14 \mathrm{MHz}$, $\Delta T=30 \mu$ s and on a device on RAC cut quartz, $f_{0}=125 \mathrm{MHz}, \Delta f=50$ $\mathrm{MHz}, \Delta T=38 \mu \mathrm{s}$. The data from $S T$ quartz agree well with our model. The data from the RAC cut device show some divergence from the simple isotropic model which predicts larger temperature dependence of chirp slope than measured. The total chirp slope change over $100^{\circ} \mathrm{C}$ for $S T$ is $400 \mathrm{ppm}$ and for RAC-cut $50 \mathrm{ppm}$.

${ }^{1}$ This work was supported by the Department of the Air Force.

JJ.4 A SAW Tapped Delay Line with Short (15-ns) Pedestal of Delay and High ( $>110 \mathrm{~dB})$ Feedthrough Isolation, ${ }^{1}$ D. E. OATES and R. W. RALSTON, Lincoln Laboratory, Massachusetts Institute of Technology, Lexington, MA 02173.

The use of very short pedestal-of-delay SAW filters has been limited because of the large direct electrical feedthrough which is unavoidable when a conventional interdigital input transducer (IDT) is positioned close to the output transducer. Feed through can be substantially reduced by using an edge-bonded input transducer (EBT) because electric fields terminate on the EBT ground plane and thereby a very effective isolation of the input with respect to the output is possible. We report here a device comprising a $\mathrm{LiNbO}_{3}$ input EBT on an $S T$ quartz substrate. A 19-finger-pair unapodized output IDT was positioned on the quartz $50 \mu \mathrm{m}$ from the EBT. At a center frequency of $120 \mathrm{MHz}$, the pedestal of delay is $15 \mathrm{~ns}$ and feedthrough isolation between input and output is $110 \mathrm{~dB}$. This low feedthrough is achieved in part by the use of a dual-track SAW design which uses a dual-electrode balanced EBT and a balanced dual-track IDT. This method of balancing provides a cancellation of the residual feed through around the EBT ground plane. Extension of this technique to a $\mathrm{LiNbO}_{3} \mathrm{SAW}$ substrate will be discussed.

${ }^{1}$ This work was supported by the Department of the Air Force.

\section{JJ-5 $250 \mathrm{MHz}$ Programmable Transversal Filter, ${ }^{1}$ C. M. PANASIK,} Texas Instruments, Dallas, TX 75266.

A programmable transversal filter is described which employs hybrid tap weight circuitry to produce continuously adjustable tap weight magnitude and sign. The $\mathrm{LiNbO}_{3}$ device consists of a conventional wideband input IDT at $250 \mathrm{MHz}$ and a 16 tap output electrode array which spatially samples the SAW at a $200 \mathrm{MHz}$ rate. A novel sampling technique allows the output array to function from 210 to $290 \mathrm{MHz}$. The dual gate FET tap weight circuitry provides over $40 \mathrm{~dB}$ of tap weight control on the biphased taps. Experimental results are compared with theoretical responses to show the bandpass and band reject capabilities of this programmable device.

1 This work was supported by Air Force Wright Aeronautical Laboratories/AAAD and Rome Air Development Center/EEA under Contract F33615-80-C-1211.

JJ-6 Cascadable Tapped Delay Line Module, D. H. WAR NE, J. J. PURCELL, and D. P. MORGAN, Plessey Research (Caswell) Limited, Allen Clarke Research Centre, Caswell, Towcester, Northants. NN12 $8 \mathrm{EQ}, \mathrm{U} . \mathrm{K}$.

This paper reports the development of a SAW tapped delay line with $120-\mathrm{MHz}$ bandwidth and $400-\mathrm{MHz}$ center frequency, using a $Y-Z$ lithium niobate substrate. The device has 42 taps with 1.0- $\mu$ spacing, giving a total delay of $42 \mu \mathrm{s}$. The taps are apodized to compensate for propagation loss and are also designed to compensate for the frequency dependence of the propagation loss. The device is designed to be cascadable so that larger delays can be obtained, and for this purpose a separate cascade channel is incorporated on the same substrate, designed to give a flat response with good spurious suppression, and with $40-\mu$ s delay. Amplifiers and trimming networks are included at the input and the cascade ou tput, so that the cascade channel has an insertion loss near $0 \mathrm{~dB}$ and can be trimmed to optimize the flatness. Experimental devices have shown good fabrication yield, with acceptable bandwidths and losses. Using trimming networks, the cascade channel response was flat to within $0.5 \mathrm{~dB}$. Experimental results will be presented, including results for several devices in cascade.

\section{MAGNETOSTATIC AND SHALLOW BULK WAVES}

\author{
Chairperson: P. H. CARR
}

KK-1 Reflection of Magnetostatic Forward Volume Waves by Ion Implanted Gratings, G. VOLLUET and P. HARTEMANN, ThomsonCSF, Research Center, Domaine de Corbeville, 91401 Orsay, France.

Physical property changes produced by ion implantation have been exploited to reflect magnetostatic forward volume waves (MSFVW) propagating in pure YIG films. For large doses, the ferrimagnetic order is broken and implanted ions induce a thin paramagnetic surface layer. Then the effective YIG thickness is reduced and MSFVW propagation is perturbed. These waves have been reflected on periodic oblique ion implanted gratings. The reflection coefficient per implanted zone boundary was determined as a function of wave number for gratings implanted with a dose of $10^{16} \mathrm{He}^{+} / \mathrm{cm}^{2}$ at $100 \mathrm{keV}$. For example it is close to 0.04 for a wavelength of $83 \mu \mathrm{m}$. An overall reflection coefficient of 40 percent has been obtained for a 15 line grating at wavelength of $140 \mu \mathrm{m}$. No noticeable insertion loss and spin wave conversion has been observed. Influence of implantation conditions on MSFVW reflection has been investigated. Two chirp reflecting arrays have been tested in a RAC configuration and preliminary results are reported.

KK-2 Magnetostatic Delay Lines using YIG Double Films, ${ }^{1}$ MICHAEL R. DANIEL and J. D. ADAM, Westinghouse Electric Corporation, Research and Development Center, Pittsburgh, PA 15235.

Propagation of magnetostatic forward or backward volume waves in two films of yttrium iron garnet coupled by their close proximity offer the promise of both dispersive or constant delay lines with wide bandwidth at microwave frequencies. The double film devices have an advantage over delay lines which utilize single films in that desirable delay characteristics can be obtained without the use of ground planes. Hence the increased insertion loss associated with magnetostatic wave damping in the ground plane is absent. This talk will describe both calculations and experimental results on "up-chirp" and "down-chirp" dispersive delay lines as well as the constant delay type.

${ }^{1}$ This work was supported in part by RADC under Contract F1962880-C-0150.

† KK-3 Magnetostatic-Wave Attenuation in One- or Two-GroundPlane Configurations, J. P. PAREKH and H. S. TUAN, Department of Electrical Engineering, State University of New York at Stony Brook, Stony Brook, NY 11794.

A dispersion control technique that has hitherto received the most attention in the studies of constant delay lines and linearly dispersive delay lines based on any one of the three magnetostatic wave-types, viz., magnetostatic surface, forward-volume and backward-volume waves (MSSW's, MSFVW's, and MSBVW's), employs the placement at an appropriate position of a single ground plane on one side of the YIG film or the placement of two ground planes one on each side of the YIG film. A deleterious feature of the ground-plane configurations is 
the MSW attenuation resulting from the ohmic dissipation in practical metal-film ground planes. The present paper is addressed to the important problem of obtaining a theoretical determination of the MSW attenuation rates in the one- and two-ground-plane configurations for practical parameters of metal-film ground planes. Quantitative MSSW, MSFVW, and MSBVW attenuation characteristics are presented and the implication in terms of practical devices are discussed.

KK-4 SAW and SSBW Propagation in Gallium-Arsenide, M. FELDMANN, J. HENAFF, and M. A. KIROV, CNET-PAB, Département MAE, 196 Rue de Paris, 92220 Bagneux, France.

III-V semiconductors are at tractive candidates for piezoelectronic devices. This paper reviews the characteristics of SAW and SSBW modes of propagation in GaAs. Starting from the known physical constants it is possible to compute the velocity and the coupling coefficent in a given configuration (cut and orientation). When accounting for possible bulk-wave contribution, we explore the leaky shells of the slowness surface. The complete atlas of all true surface waves has been computed, and the results will be discussed. Gallium arsenide remains a rather low coupling material, the $k^{2}$ coefficient culminating at roughly 0.09 percent for the true surface branch. This mode is a pure Rayleigh mode, which turns out to be leaky in the vicinity of this configuration. In order to find out larger coupling, we have also computed the characteristics of the first leaky shell of the slowness surface, and selected results will be reported. For example, we have found a SSBW branch with 0.11 percent coupling and negligible attenuation. Finally, the interest of $\mathrm{GaAs}$ for piezoelectronics and signal processing will be discussed.

KK-5 Observations on Surface Skimming Bulk Waves and Other Waves Launched from an IDT on Lithium Niobate, P. D. BLOCH, N. D. DOE, E.G.S. PAIGE, and M. YAMAGUCHI, Department of Engineering Science, University of Oxford, Parks Road, Oxford, OX1 3PJ, England.

The high value of $k^{2}$ associated with shear horizontal waves launched by an interdigital transducer (IDT) on $41^{\circ}$-rotated $Y$-cut lithium niobate (an SSBW cut) makes the launching the detection of these waves of particular interest. Measurements of frequency response and impulse response show that two electrically active waves propagate close to the surface. Under a water or metal layer the velocity of one wave is not detectably affected but the other is slowed by 5 percent under water and 10 percent under metal. Theoretical calculations are in good agreement with the experimental observations and lead to the interpretation of the wave slowed by the layer as a leaky SAW and the unaffected wave as an SSBW. Predicted and observed attenuation are also in fair agreement. Strong radiation of shear vertical waves into the bulk of the crystal from the IDT and from metal edges is observed by optical probing.

KK-6 A General Theory for the Interaction of Bulk and Surface Acoustic Waves on Piezoelectric Substrates, F. JOSSE, D. L. LEE, and J. F. VETELINO, Department of Electrical Engineering and Laboratory for Surface Science and Technology, University of Maine, Orono, MA 04469.

A general theory appropriate to describing the excitation of both bulk and surface acoustic waves in piezoelectric media is presented. In particular, the theory is capable of treating those orientations in which propagating bulk wave and SAW velocities are in close proximity. It is shown that under such conditions the well known parameter, $\Delta v / v$, is no longer a valid measure of the coupling coefficient for the surface wave. The theory is particularly relevant to high frequency SSBW devices fabricated on quartz and berlinite in which mass loading effects from metal electrodes can cause significant conversion of bulk wave radiation into a Love-type surface wave underneath the transducers. The analysis is also applicable to SAW filter design in which spurious bulk responses must be minimized. Closed form expressions are derived for the transducer radiation resistance, surface potential, and insertion loss in terms of transducer geometry and only four substrate parameters, the bulk wave cutoff velocity, the open and short circuit surface wave velocities, and the off-resonance substrate dielectric constant. The theory is compared to experimental measurements of energy trapping effects made on rotated $Y$-cut quartz.

\section{MEDICAL ULTRASOUND: HYPERTHERMIA AND THERMAL}

\author{
Chairperson: L. A. FRIZZELL
}

†LL-1 An Annular Focus Ultrasonic Lens for Local Hyperthermia Treatment of Small Tumors, ${ }^{1}$ R. E. BEARD ${ }^{2}$ R. L. MAGIN, L. A. FRIZZELL, and C. A. CAIN, Bioacoustics Research Laboratory, Department of Electrical Engineering, University of Illinois, 1406 West Green Street, Urbana, IL 61801.

A system for applying local hyperthermia employing ultrasonic transducers with annular focusing lenses gave better temperature uniformity in small fibrosarcomas than conventional unfocused transducers. The lenses were designed for ultrasonic frequencies of $3,4,5$, and $9 \mathrm{MHz}$ for tumors approximately $6-\mathrm{mm}$ in diameter. Tests in degassed water indicated that the focusing lenses concentrated approximately 80 percent of the ultrasonic energy into an annular-shaped focus of 5- and 6-mm in diameter, located at a distance of about 2.5 to $3 \mathrm{~cm}$ in front of the lens. These transducers were used to heat subcutaneous PARA-7 fibrosarcomas locally in hamsters. Steady state temperature measurements indicated temperature gradients within the tumors of 0.5 to $1.0^{\circ} \mathrm{C}$ when the transducers employing annular-focused lenses were used versus gradients of 2.0 to $2.3^{\circ} \mathrm{C}$ with $10-\mathrm{mm}$ diameter unfocused transducers.

\footnotetext{
${ }^{1}$ This work was supported in part by the National Institutes of Health.

${ }^{2}$ Present address: Battelle Columbus Laboratories, 505 King Avenue, Columbus, $\mathrm{OH} 43215$.
}

LL-2 Annular Array Transducer for Deep Acoustic Hyperthermia, J. P. DO-HUU and P. HARTEMANN, Thomson-CSF, Research Center, Domaine de Corbeville, 91401 Orsay, France.

In order to achieve acoustic hyperthermia of biological tissues located at a great depth from the skin $(12 \mathrm{~cm})$, an annular array transducer operating at $400 \mathrm{kHz}$ was performed. This array is made of $14 \mathrm{con}$ centric ring elements fed by voltages with suitable phase and magnitude to obtain a discontinuously variable focal distance. Acoustic power distribution in abscrbing propagation medium was computed for different element widths and spacings between adjacent rings. Then the transducer configuration has been optimized for hyperthermia. An increase of the heated tissue volume was investigated by adjusting the relative phases of driving voltages. Such a transducer was realized with a PZT disc of $100-\mathrm{mm}$ in diameter. Acoustic coupling between rings has been measured for three values of the depth of grooves separating the elements and the resonance frequency of elementary transducers has been studied versus width to thickness ratio. The equipment is capable of launching an acoustic power of $50 \mathrm{~W}$. Profiles of sound pressure in a water bath and temperature distributions in phantoms have been determined for different focal distances and apertures.

LL-3 Some Experimental Results on the Thermoacoustic Imaging of Tissue Equivalent Phantom Materials, T. BOWEN, R. L. NASONI, A. E. PIFER, and G. H. SEMBROSKI, Department of Physics and Division of Radiation Oncology, University of Arizona, Tucson, AZ 85724. 
The use of the thermoacoustic emissions from ionizing or nonionizing radiation in tissue holds promise as a valuable aid in radiation or hyperthermic therapy as well as an alternate and possibly complementary mode of diagnostic imaging. The present paper reports on a) the experimental verification of certain of the theoretical predictions concerning thermoacoustic imaging and $b$ ) the practicality of using signal averaging techniques to obtain images. Thermoacoustic waves were induced in soft tissue phantoms consisting of layers of muscle tissue phantom gel and vegetable oil by $0.4-\mu$ s duration electric current pulses. The acoustic signals were detected by digitally averaging $10^{3}$ to $10^{4}$ waveforms. The observed signals had the amplitude, time-delay, and signal-to-noise ratio characteristics expected for thermoacoustic emission from soft tissues. The results support the feasibility of thermoacoustic imaging of soft tissue by a transducer-amplifier-averaging system optimized for this application.

LL-4 Ultrasonic Heating Distribution in Lossy Cylinders and Spheres, L. A. LONGLEY ${ }^{1}$ and W. D. O'BRIEN, JR., Bioacoustics Research Laboratory, Department of Electrical Engineering, University of Illinois, 1406 West Green, Urbana, IL 61801.

The distribution of heat generation by viscous loss in infinitely long cylinders and spheres is estimated from the lossless acoustic wave distributions resulting from an incident planewave. It is assumed that each object has a uniform, lossy interior, similar to tissue, and that it is surrounded by an infinite lossless material with different acoustical properties, similar to water. Impedance mismatches of up to 20 percent are considered for normalized sizes, ka, which ranged from 1 to 40 . Plots of typical heat-generation distributions are presented, and apparent trends are summarized. The greatest spatial nonuniformity and highest peaks of heat generation were found for maximum ka and maximum impedance mismatch.

${ }^{1}$ Current Address: Motorola, Inc., 1301 E. Algonquin Road, Schaumberg, IL 60196.

$\dagger$ LL-5 Development of a Phantom Tissue Material for Hyperthermia, F. E. BARBER, D. PLENKOVIC, D. AJGAONKAR, and K. KASE, Harvard Medical School and the Sidney Farber Cancer Institute, 44 Binney St., Boston, MA 02115.

The three-component fluid system, water-alcohol-glycerine, can be tailored to yield a number of combinations of velocity, temperature coefficient of velocity, and absorption useful for ultrasonic phantoms. Using a specially developed sample chamber, the pulse-echo overlap method has been adapted to measure these parameters throughout the physiologic temperature range from 25 to $45^{\circ} \mathrm{C}$. Some initial results are plotted on triangular three phase diagrams such that desired combinations of properties can be selected as the intersection of contours of constant individual properties. The system has been used with band-limited pulses centered at $2.25,3.5$, and $5 \mathrm{MHz}$. Temperature accuracy is $0.2^{\circ} \mathrm{C}$ and velocity accuracy is 0.2 percent. Accuracy of absorption measurements has not been assessed at this time. Selected fluid mixtures are used in a solid-fluid scatter matrix containing a hydrophyllic polymer for the solid component. The primary objective is a tissue equivalent phantom for hyperthermia.

LL-6 Determination of Threshold Parameters for Cutaneous Thermal Necrosis from Ultrasonic Bum Depth Measurements, JOHN H. CANTRELL, JR., NASA Langley Research Center, Hampton, VA 23665 and RONALD E. GOANS, George Washington University, Washington, DC 20052.

Knowledge of the in vivo threshold parameters for thermally induced dermal necrosis is important to the understanding and assessment of the total burn injury. We have developed an ultrasonic pulse-echo system for assessing thermal injury and have used the system to obtain quantitative in vivo measurements of the depth of cutaneous burns ex perimentally induced in anesthetized Yorkshire pigs. The burn depth as a function of burn time and surface temperature of the pig skin was measured. The data were used in the solution of the one-dimensional heat diffusion equation with time-dependent boundary conditions to obtain the threshold temperature $\left(65.3^{\circ} \mathrm{C}\right)$ and the energy of phase transformation $\left(886 j \mathrm{gm}^{-1}\right)$ associated with the transition of the tissue from a state of viability to a state of necrosis. In addition, we have combined the present results with previous measurements of the accumulated damage rate process to calculate the molecular weight $(710$ Daltons) of the chemical constituent of the cell whose thermally induced inactivation is responsible for cellular death at the threshold level.

\section{SAW PROPAGATION PHENOMENA}

\section{Chairperson: T. J. LUKASZEK}

MM-1 Solitons in Surface Acoustic Wave Media, ${ }^{1}$ J. F. EWEN and R. L. GUNSHOR, School of Electrical Engineering, and V. H. WESTON, Division of Mathematical Sciences, Purdue University, West Lafayette, IN 47907.

Recently, in the area of mathematical physics, there has been a growing interest in nonlinear, dispersive systems, and particularly in solitons, a type of wave which exists as a balance between the nonlinearity and the dispersion. There are two basic types of solitons, one of which is a baseband, pulse-like signal and the other consisting of an RF pulse in which the envelope exhibits soliton behavior. In both cases the soliton behavior is characterized by the stable propagation of the signal in the presence of nonlinearity and dispersion. All SAW devices exhibit dispersion and nonlinearity to varying degrees. The purpose of this paper is to relate the rapidly growing developments in soliton theory to the SAW device field. Computer simulations of a phenomenological model identify various parameters which characterize the development of an initial condition into solitons. Experimental measurements of harmonic generation and pulse propagation are used to establish the validity of the model. A number of SAW device configurations are evaluated in the context of the proposed model.

${ }^{1}$ This work supported by NSF-MRL Grant DMR 77-23798.

†MM-2 New Orientation of Lithium Niobate for Low Bulk-Wave Degradation of SAW Filter Stopband, R. F. MILSOM, R. J. MURRAY, and I. FLINN, Philips Research Laboratories, Redhill, Surrey RH1 5HA, England, M. REDWOOD, Department of Electrical and Electronic Engineering, Queen Mary College, London El 4NS, England.

Degradation of the stopband by spurious bulk-wave signals is a major problem in the design of SAW bandpass filters. A number of schemes exist for reducing the problem but all involve a penalty in terms of production cost. Low bulk-wave substrate orientations have been previously sought, but incorrect criteria have led to nonoptimum cuts. We have recently ${ }^{1}$ reported a new range of cuts of lithium niobate for which bulk-wave signals are weakest at the frequencies where maximum suppression of spurious level is required. Choice of cut angle is shown to be a function of bandwidth and shape factor. In this paper we present more details of the theoretical basis of this choice and the results of more extensive experimental verification.

${ }^{1}$ R. F. Milsom, R. J. Murray, I. Flinn, and M. Redwood, "Ultra low bulk orientations of lithium niobate for SAW TV filters," Electron. Lett., vol. 17, pp. 89-91, 1980.

MM-3 Diffraction in SAW Multistrip Beam Compressors, H. ENGAN, K. A. INGEBRIGTSEN, and A. RØNNEK LEIV, Norwegian Institute of Technology, N-7034 Trondheim-NTH, Norway. 
SAW multistrip couplers provide an efficient way of compressing wide aperture acoustic beams into narrow SAW waveguides. Efficient single mode excitation of the waveguide requires control with the spatial phase and amplitude profile of the compressed beam. Laser probe measurements have verified that diffraction in the multistrip coupler contributes significantly to the resulting profile. The paper presents a model for diffraction in multistrip beam compressors. Numerical calculations on a nominally $15: 1$ compressor designed for a 5 wavelength SAW metal strip waveguide are compared with laser probe measurements.

$\uparrow$ MM-4 Piezoelectric Glass-Ceramics, ${ }^{1}$ A. HALLIY AL, A. S. BHALLA, L. E. CROSS, ${ }^{2}$ and R. E. NEWNHAM, Materials Research Laboratory, Pennsylvania State University, University Park, PA 16802.

Polar glass ceramics were prepared by recrystallization of glasses of various compositions in the systems $\mathrm{BaTiO}_{3}-\mathrm{GeO}_{2}$ and $\mathrm{Li}_{2} \mathrm{O}_{-} \mathrm{SiO}_{2}$ $\mathrm{B}_{2} \mathrm{O}_{3}$ in a temperature gradient. The electromechanical properties of the piezoelectric elements of the glass ceramics were studied, and the resonance properties and their temperature dependence were evaluated. Piezoelectric elements of certain compositions in $\mathrm{Li}_{2} \mathrm{O}-\mathrm{SiO}_{2}-\mathrm{B}_{2} \mathrm{O}_{3}$ show strong radial coupling and low temperature dependence of the resonance properties. Such systems may have application in SAW devices. Under controlled crystallization a desired thickness of the thin glass plate can be crystallized into a glass-ceramic layer and such composite elements may be useful for device applications based on flexural resonance modes.

' This work was sponsored by the U.S. Army Research Office (DAAG29-80-C-0008).

${ }^{2}$ Also affiliated with the Department of Electrical Engineering.

MM-5 Electronically Variable Surface Acoustic Wave Time Delay Using a Biasing Electric Field, ${ }^{1}$ S. G. JOSHI and B. B. DASGUPTA, Marquette University, Milwaukee, WI 53233.

The time delay of surface acoustic waves can be varied by applying a biasing electric field to the propagation medium. ${ }^{1}$ In this paper, we will present results of experiments performed on a number of different materials. These include single crystals $\left(\mathrm{LiNbO}_{3}, \mathrm{LiTaO}_{3}\right.$, and quartz) as well as poled ferroelectric ceramics (PZT and modified $\mathrm{PbTiO}_{3} \mathrm{ce}-$ ramics). Fractional changes in time delay up to $5 \times 10^{-4}$ appear practical in $\mathrm{LiNbO}_{3}$ devices. It will be shown that the changes in time delay that can be obtained are adequate to make the technique useful for several applications. Potential applications that will be discussed are 1) beam steering in phased array antennas, 2) direct voltage-to-frequency conversion, and 3) compensating against temperature and aging effects in SAW devices. A rigorous formalism for calculating the change in velocity of surface acoustic waves due to the presence of external stress or electrostatic field has been given by Tiersten. We propose a simpler theory, similar to the one developed by Nalamwar and Epstein, which is easier to understand physically, but retains most of the mathematical rigor of Tiersten's formulation. Results obtained from this theoretical model are compared with our experimental results.

${ }^{1}$ S. G. Joshi, Proc. 1980 Ultrasonics Symposium, pp. 438-441.

MM-6 Tunable ZnO-Si SAW Devices Using Magnetostrictive Thin Films, ${ }^{1}$ BIROL ALTAN and WILLIAM P. ROBBINS, Department of Electrical Engineering, University of Minnesota, Minneapolis, MN 55455 .

Modestly tunable ( $\Delta V / V \sim .01$ to 0.1 percent) $\mathrm{ZnO-on-Si} \mathrm{SAW} \mathrm{delay}$ lines have been fabricated by RF sputtering magnetostrictive films on the surface of $\mathrm{ZnO}$ thin films on silicon. Two types of magnetostrictive films have been used including $\mathrm{Tb}-\mathrm{Fe}$, a magnetically hard material, and Fe-Si-B, a magnetically soft material. Relative velocity changes, $\Delta V / V$, of 0.02 percent at $140 \mathrm{MHz}$ have been observed with 1 micron thick Fe-Si-B films at fields of less than 200 Oe while $\Delta V / V$ of 0.03 percent have been measured at $130 \mathrm{MHz}$ with 1 micron thick Tb-Fe films at fields of 5 kilogauss. Untuned zero field insertion losses were typically 50 to $60 \mathrm{~dB}$ with 5 to $10 \mathrm{~dB}$ being accounted for by the magnetostrictive thin films. Further improvements in $\Delta V / V$ and lower insertion losses are expected as optimum deposition conditions are determined. Additionally the alternative film structure of magnetostrictive film on silicon and then $\mathrm{ZnO}$ deposited on top of the magnetostrictive film may have some utility and is currently being fabricated.

${ }^{1}$ This work was partially supported by the National Science Foundation.

\section{NDE MATERIAL CHARACTERIZATION}

\section{Chairperson: K. M. LAKIN}

NN-1 Effective Properties of Perforated Solids Including Effects of Ultrasonic Resonances, G. C. GAUNAURD, and H. ÜBERALL, ${ }^{1}$ Naval Surface Weapons Center, White Oak, Silver Spring, MD 20910.

It is well-known that in the static case, (visco) elastic media containing random microinhomogeneities may be characterized by effective moduli. In the ultrasonic regime, resonances of the microinhomogeneities appear which require a description in terms of dy namic effective moduli. We have developed an analy tic approach of modeling the microinhomogeneities deterministically, by extending the static AmentToksöz method to to the resonant case. This is achieved by retaining those higher order terms in a long-wavelength expansion that give rise to the resonances. The present analysis contains the results of our previous dy namic analysis of gas bubbles in liquids, as well as all static results given in the earlier literature that do not include rescattering effects (an assumption used also in the present work, which renders the results applicable for moderated volume concentrations $\lesssim 10$ percent only).

${ }^{1}$ Also at Department of Physics, Catholic University, Washington, DC; supported by Office of Naval Research, Code 421 .

NN-2 Characterization of Heat Treatment in Aluminum Based on Ultrasonic Determination of the Second and Third Order Elastic Constants, JOSEPH S. HEYMAN and ENGMIN J. CHERN, ${ }^{1}$ NASA Langley Research Center, Hampton, VA 23665.

Recent processing errors by suppliers of high strength aluminum alloys have resulted in the possible placement of sof $t$ aluminum in critical structures such as the space shuttle. This paper discusses a nondestructive technique having potential for evaluating structural members in place, thus assuring initial structural viability as well as locating regions of material suffering annealing resulting from reentry or from processing irregularities. Although investigations of ultrasonic velocity indicate its insensitivity to material heat treatment, only second order elastic constants have been considered. In this study, we show that combination of second and third order elastic constant measurements offer the potential of characterizing aluminum heat treatment. Measurements of material hardness, conductivity, and ultimate strength in a 2024 aluminum alloy are compared to both stress and thermal acoustic constants. Ultrasonic measurements presented were obtained with a pulsed phase locked loop ultrasonic spectrometer with resolution of parts in $10^{7}$ and exhibit significant variations in ultrasonic properties (greater than 20 percent) with heat treatment.

\footnotetext{
${ }^{1}$ Research Associate, College of William and Mary, Williamsburg, VA
} 23185 . 
NN-3 The Ultrasonic Properties of Impregnated Graphite, ${ }^{1}$ J. F. MURATORE and H. R. CARLETON, College of Engineering and Applied Sciences, State University of New York, Stony Brook, NY 11794.

The ultrasonic properties of graphite have been evaluated in the intrinsic state and after impregnation with several liquids. The density and velocity of this material is found to vary with the degree of saturation and liquid characteristics. The apparent ultrasonic velocity of graphite with open pores is lower than that of the crystalline phase. When the voids are filled, the velocity increases and the effects of scattering are reduced, presumably due to the reduction in acoustic inhomogeneity. The damping and acoustic impedance can be controlled over modest ranges by the selecting of liquid properties and graphite porosity. Since the acoustic impedance of this material is in the range of $7 \times 10^{6}\left(\mathrm{~m}^{2} \mathrm{~s}\right)^{-1}$ it has interesting potential in the design of wideband transducers.

${ }^{1}$ This work has been funded under the management of the New York State Energy Research and Development Authority.

NN-4 Ultrasonics in Determining Characteristics of Coal, ${ }^{1}$ W. J. HADDEN, JR., Department of Mechanical Engineering, University of Texas at Austin, Austin, TX 78712, and C. H. CHEW, Department of Mechanical Engineering, University of Singapore, Singapore, Republic of Singapore.

This paper presents results of an experimental and analy tical investigation of sound speeds and attenuation rates in bituminous coal in the ultrasonic frequency range. The experimental phase employed a simple water-bath immersion technique. Values of the sound speed and attenuation factor determined from these tests compare well with predictions based on an analytical model of coal as a transversely isotropic anelastic medium with randomly distributed fluid-filled inclusions. Numerical investigations using this model indicate that the attenuation rate, in particular, is a sensitive function of the concentration of pores in the coal, as well as of their aspect ratio. A study of the variation of the sound speed and attenuation rate with time of immersion shows that these could be used to determine the extent of saturation with water or other fluids. The utility of sound speed measurements as an adjunct to densimetric methods for determining the chemical composition of coal is shown by computation of a molar sound speed.

${ }^{1}$ This work was supported in part by NASA Marshall Space Flight Center, Huntsville, AL.
NN-5 Dynamic Monitoring of the Precipitation Hardening Process in 2219 Aluminum Alloy by Means of Sound Velocity and Attenuation Measurements, M. ROSEN and E. HOROWITZ, Center for Materials Research, The Johns Hopkins University, Baltimore, MD 21218 and S. FICK and R. MEHRABIAN, Metallurgy Division, National Bureau of Standards, Washington, DC 20234.

The precipitation hardening process in 2219 aluminum alloy has been investigated by means of dy namic measurements of sound velocity, ultrasonic attenuation, and hardness. Measurements of these properties as a function of aging time at constant temperatures $(150,175$, and $220^{\circ} \mathrm{C}$ ) were found to exhibit prominent variations that were related to the formation of precipitates responsible for the hardening process. Dynamic ultrasonic measurements, corroborated, a posteriori, with hardness and electron microscopy examination enabled identification of the loss of coherency of precipitates with the matrix resulting in softening of the alloy. The frequency dependence of the ultrasonic attenuation is suggestive of a relaxational mechanism related to the size and distribution of the precipitates. The present investigation has demonstrated the feasibility of a real-time dy namic NDE method for monitoring the progress of the precipitation hardening process over a wide temperature interval.

NN-6 Utrasonic Imaging of Material Mechanical Properties Through Steel Pipe, B. FROELICH, A. DUMONT, D. PITTMAN, and B. SEEMAN, Etudes et Productions Schlumberger, 92140 Clamart, France.

A technique is described for the measurement of material acoustic impedance outside a steel pipe with access limited to the inside. The problem addressed is the quality control of the cementing process in cased oil wells. The physical environment is especially hostile with high temperature and pressure (up to $175^{\circ} \mathrm{C} / 1400$ bars) and at tenuating fluids, such as drilling muds. The principle is to excite the pipe thickness resonance with a short ultrasonic pulse around $500 \mathrm{kHz}$. From the reflected pulse one can extract the resonance which has a rate of decay related to the acoustic impedance of the medium behind the steel wall. An empirical relation has been established between the acoustic impedance of cement and its compressive strength; so that by probing the pipe surface with eight multiplexed transducers at $45^{\circ}$ from each other, it is possible to map the cement quality along the well with good vertical and azimuthal resolutions. Field examples with typical cement defects are discussed. 